\title{
A Biomechanical Comparison of Repair Techniques for Complete Gluteus Medius Tears
}

\author{
By \\ JUSTIN DISHKIN-PASET \\ BSE, University of Iowa, 2009
}

\section{THESIS}

Submitted as partial fulfillment of the requirements

for the degree of Master of Science in Bioengineering in the Graduate College of the

University of Illinois at Chicago, 2011

Chicago, Illinois

Defense Committee:

Thomas Royston, Chair, Department of Bioengineering Vincent Wang, Advisor, Department of Bioengineering Shane Nho, Department of Orthopedic Surgery, Rush University Medical Center 


\section{ACKNOWLEDGEMENTS}

I would like to thank my advisor, Dr. Vincent Wang, whose constant guidance along friendship as lead me to where I am today. Without your education, I would not fully understand all that goes into research and appreciate that my thoughts and ideas do have an impact. It was truly an honor to work with you.

I would like to thank Dr. Shane Nho and Smith \& Nephew for the research funds for this study. To Dr. Nho, I thank you for your patience and guidance in teaching me about the hip. It was a wonderful experience to have with you.

I am honored to work with Elizabeth Shewman, who has helped me from the start and has never been too busy to answer any of my questions.

I appreciate all the hard work on this study from Michael Salata, Katie Manno, and Chris Gross.

I would like to acknowledge my colleagues, Rebecca Bell and Aman Gupta along with my other coworkers for their support and friendship.

Finally, I owe all that I am today to my family. They are proud of all my accomplishment and I hope we experience many more. I appreciate their undying love and support. 


\section{TABLE OF CONTENTS}

Section 1: Introduction 1

Gluteus Medius Anatomy

Classification of Gluteus Medius Tears 2

"Parallels" to the Shoulder 4

$\begin{array}{lc}\text { Surgical Intervention } & 6\end{array}$

Recent Gluteus Medius Findings

Tendon 9

Motivation and Objective 12

Section 2: Methods 14

$\begin{array}{ll}\text { CT Scan } & 14\end{array}$

$\begin{array}{ll}\text { Specimen Preparation } & 15\end{array}$

Biomechanical Testing 20

Data Analysis 23

Section 3: Results 31

Anatomical Data

Mechanical Data: Intact Group 32

Mechanical Data: Repair Groups 37

Optical Data: Repair Group

Correlation Factor $\quad 44$

Section 3: Discussion 46

Cited Literature $\quad 55$

$\begin{array}{ll}\text { Appendix } & 58\end{array}$

$\begin{array}{ll}\text { Vita } & 78\end{array}$ 


\section{LIST OF TABLES}

Table 2.1 Specimen details and groupings....................................... 16

Table 3.1 Anatomical Data for Repair Groups and Intact Specimens.....................32

Table 3.2 Mechanical Data for the Intacts ..........................................

Table 3.3 Curve fit data (coefficients A,B,and C) and Young's Modulus ...................34

Table 3.4 Exponential Coefficients for other tissues measured ........................34

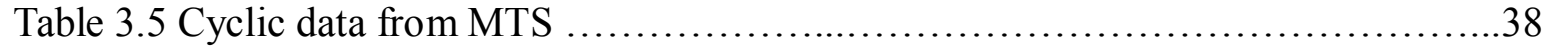

Table 3.6 Mechanical Data of the Repair Groups .................................... 39 


\section{LIST OF FIGURES}

Figure 1.1 Gluteus Medius muscle from origin to insertion site $\ldots \ldots \ldots \ldots \ldots \ldots \ldots \ldots \ldots \ldots \ldots . \ldots \ldots$

Figure 1.2 Digitized insertional footprint of muscles on the greater trochanter.................2

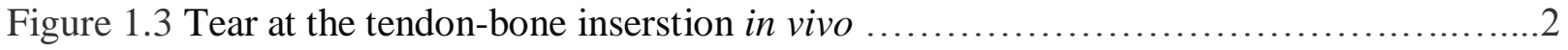

Figure 1.4 Oval shaped tear, where the underlying bone can be seen ........................

Figure 1.5 Rotator cuff repair using two kinds of anchors and a mattress stitch................5

Figure 1.6 Rotator cuff repair using an anchor and two kinds of stitches $\ldots \ldots \ldots \ldots \ldots \ldots \ldots \ldots . . .5$

Figure 1.7 Arthroscopic image of suture anchors used to repair tendon in vivo....................7

Figure $1.8 \mathrm{GM}$ tendon sutured to the insertion site by anchors in vitro.....................

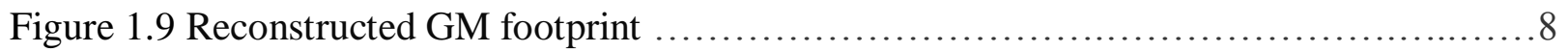

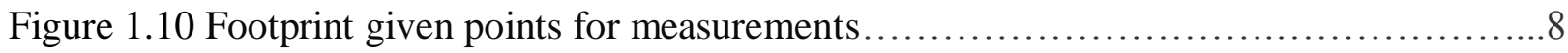

Figure 1.11 Patient based MRI images after successful GM repair .........................9

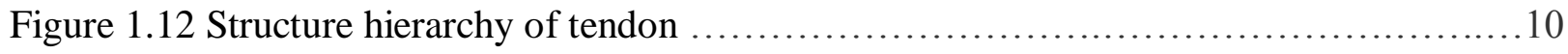

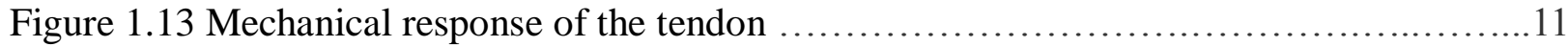

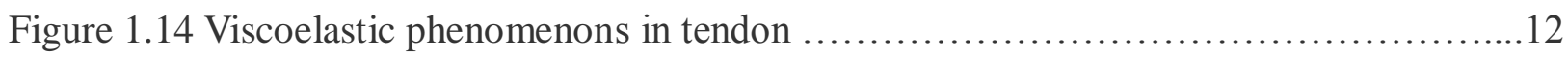

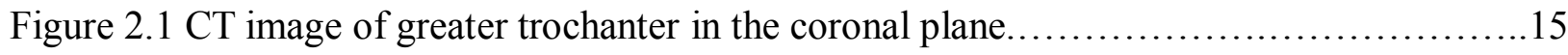

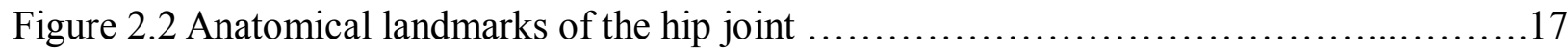

Figure 2.3 Entire GM muscle-tendon excised at insertion point $\ldots \ldots \ldots \ldots \ldots \ldots \ldots \ldots \ldots \ldots \ldots 18$

Figure 2.4 Outlined Lateral and Superoposterior footprint $\ldots \ldots \ldots \ldots \ldots \ldots \ldots \ldots \ldots \ldots \ldots \ldots 18$

Figure 2.5 Schematic of (a) DR-MCS and (b) DR-KLA .................................. 19

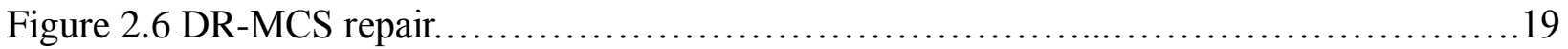

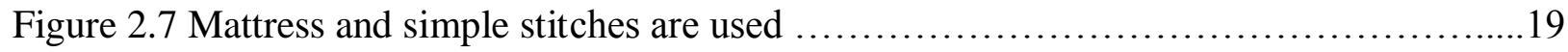

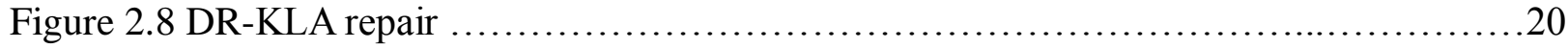




\section{LIST OF FIGURES (continued)}

Figure 2.9 Knotless anchors with sutures placed into the bone just below tendon ............20

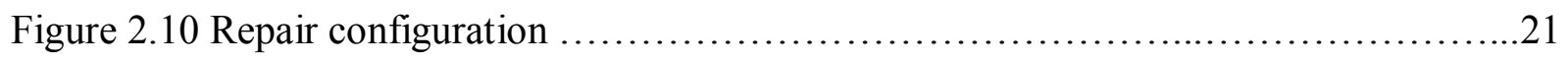

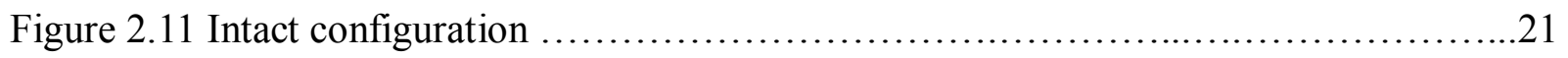

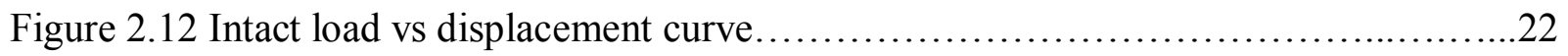

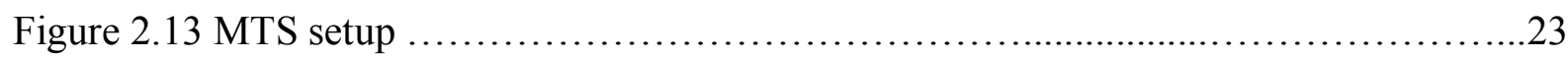

Figure 2.14 Representative results from cyclic testing of repaired construct .................24

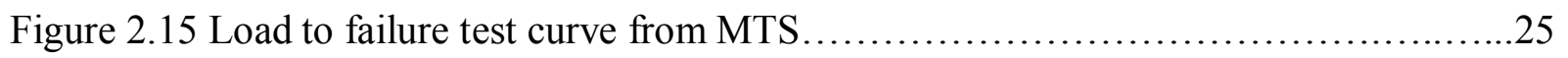

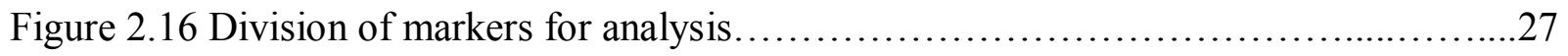

Figure 2.17 Marker placement and defined regions for optical analyses....................27

Figure 2.18 Original curve of specimen from MatLab................................29

Figure 2.19 Curve after linear regression analysis .................................29

Figure 2.20 Exponential Curve Fitting ........................................ 30

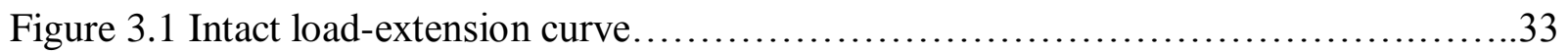

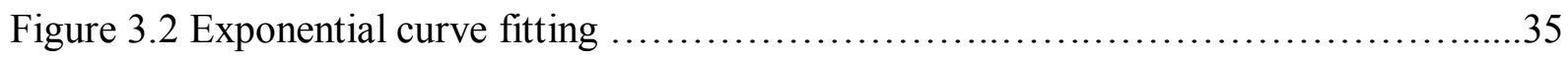

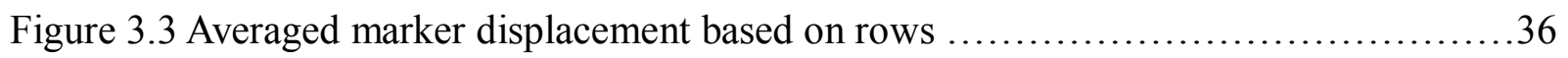

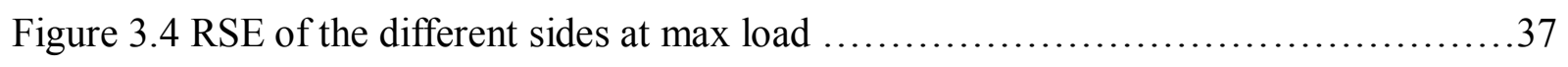

Figure 3.5 Repair curves....................................................... 39

Figure 3.6 DR-MCS failure occurred by suture pulling through the tendon .................40

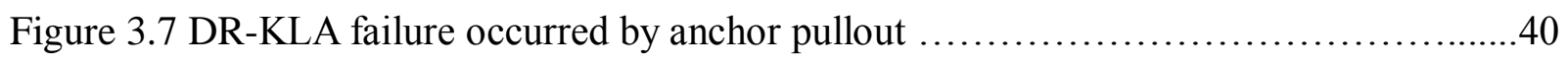

Figure 3.8 Slopes of the (a) DR-KLA and (b) DR-MCS repairs ...........................

Figure 3.9 An optical data sample of the RSE for the RTU.......................................42

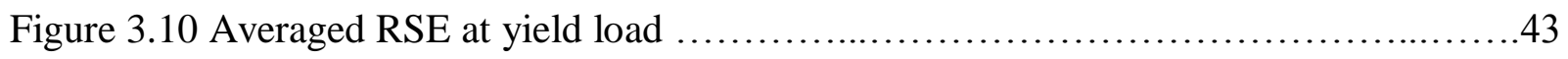




\section{LIST OF FIGURES (continued)}

Figure 3.11 Sample optical data of the post yield extension for the RTU ..................43

Figure 3.12 DR-KLA and DR-MCS correlation of $\max$ load......................... 44

Figure 3.13 DR-KLA and DR-MCS correlation of tendon thickness......................45 


\section{SUMMARY}

Lateral hip pain has been associated with tears of the gluteus medius muscle. When conservative management fails, repair is necessary. However, biomechanical properties of repair constructs for gluteus medius tendon tears have not previously been described. In the present study, sixteen fresh-frozen human cadaveric hemi-pelves were tested. Bone mineral density and anatomical measurements were evaluated. Six specimens received Double Row with Massive Cuff Stitch (DR-MCS) repair, six received Double Row with Knotless Lateral Anchors (DR-KLA), and four remained intact. Markers were placed on the tissue for video tracking. Intact specimens were preloaded to $10 \mathrm{~N}$ and loaded to failure at $1 \mathrm{~mm} / \mathrm{sec}$ to establish a cyclic testing protocol for the repair groups. Repaired specimens were preloaded to $10 \mathrm{~N}$, loaded from $10 \mathrm{~N}$ to $125 \mathrm{~N}$ at $90 \mathrm{~N} / \mathrm{s}$ for 150 cycles, and then loaded to failure at $1 \mathrm{~mm} / \mathrm{sec}$. The superoposterior footprint length was found to be significantly greater than the lateral footprint length. Cyclic segment elongation was significantly higher for the DR-MCS than the DR-KLA. The DR-KLA showed a significantly higher $(\mathrm{p}<0.05)$ normalized yield load than the DR-MCS. Linear stiffness and post yield extension for the DR-MCS were significantly higher than the DR-KLA. At yield load, the optically measured soft tissue elongation of the DR-KLA was significantly greater than the DRMCS. Mechanical properties of the selected repair constructs were inferior to those of the intact unit. The DR-KLA repair exhibited its first signs of detachment closer to the point of complete failure by anchor pullout, while the DR-MCS repair showed initial failure earlier by suture rupture. Bone mineral density was strongly correlated to maximum load for the DR-KLA repair. Anatomical data from patients are vital to the selection of an appropriate repair method, in which BMD must be taken into account. Results indicate that both repair constructs have adequate mechanical integrity to withstand early rehabilitation post-operatively. 


\section{Section 1: Introduction}

Gluteus Medius Anatomy

The gluteus medius (GM) muscle's primary function is to abduct the thigh and rotate it medially. This muscle rests superior to the gluteus minimus, where both muscles are fused at their anterior borders. The origin is on the pelvis; specifically, on the outer surface of the ilium near the iliac crest (Figure 1.1). The muscle portion is very broad, spanning the entire ilium. The insertion site is located on the greater trochanter on the femur ${ }^{1}$. The greater trochanter arises from the junction of the femoral neck and shaft. There are 5 muscles that attach to the greater trochanter, where the gluteus medius and gluteus minimus tendons are located on the lateral surface and the piriformis, obturator externus and obturator internus are more medially ${ }^{3}$.

Based on other studies (described below), the insertion site on the greater trochanter is divided into two bony attachments (Figure 1.2). First, muscle fibers will attach to the superoposterior portion or facet of the greater trochanter, where its orientation is in line with the femoral neck. Muscle fibers will also attach to the lateral facet near the bald $\operatorname{spot}^{2}$. The bald spot is defined as a portion of the greater trochanter that has no soft tissue attachments and represents the location of the subgluteus medius bursa. The sectioning of the insertion or footprint has made surgical intervention complex because the tendon needs to be repaired over the original footprint. The nerve supply of the muscle is the superior gluteal (L4, L5, and S1) ${ }^{1}$. 


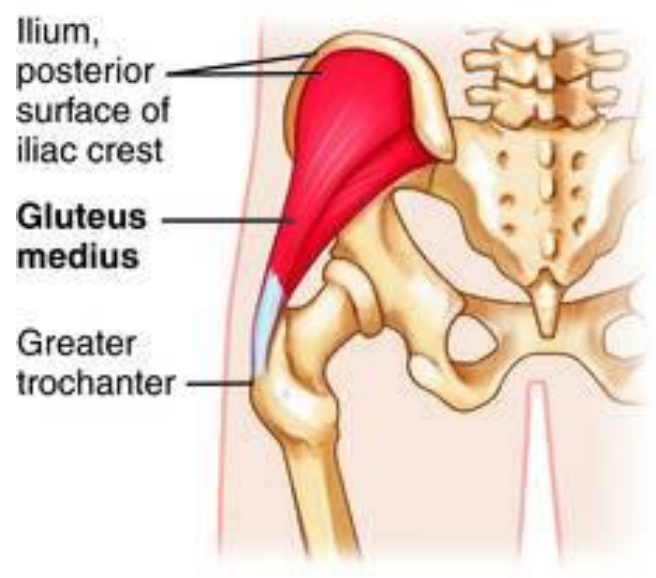

Figure 1.1- Gluteus Medius muscle from origin to insertion site. (Medical dictionary)

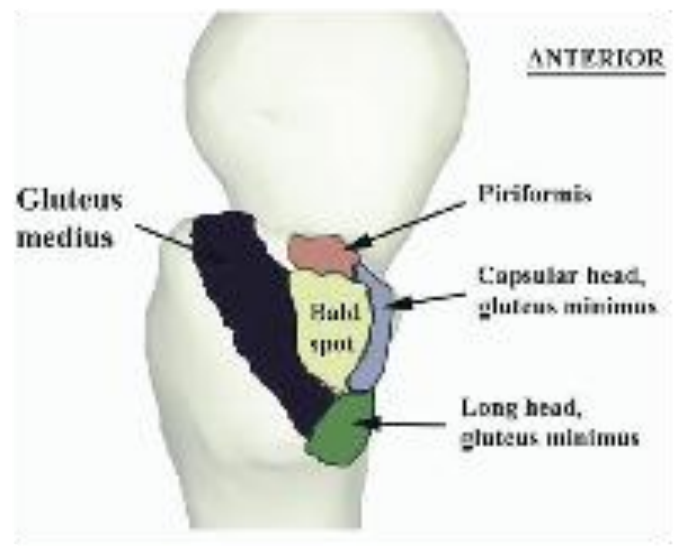

Figure 1.2- Digitized insertional footprint of muscles on the greater trochanter ${ }^{2}$.

\section{Classification of Gluteus Medius Tears}

Recently, there have been reports of an increased onset of lateral hip pain in patients, which is associated with tears of the $\mathrm{GM}^{2,3,4,5}$. These tears commonly occur at the insertional footprint of the GM tendon (Figure 1.3). Although the reasons for these tears are unknown, it is suggested that tears will develop in late middle-aged women and men. Furthermore, these tears occur 4 times more frequently in women than in men, yet the incidence increases at a similar rate in both groups ${ }^{2}$. The vast majority of patients with symptomatic tears describe a gradual onset of lateral-sided hip pain.

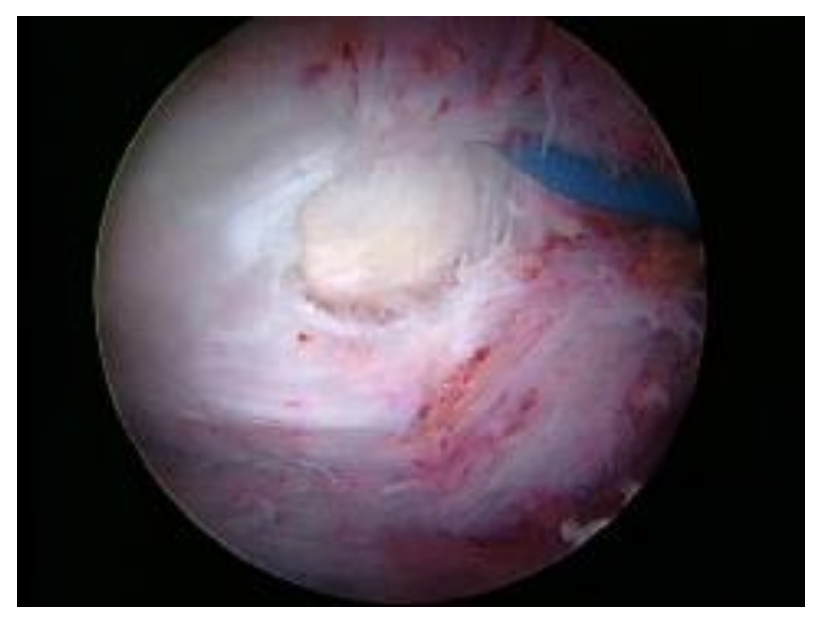

Figure 1.3- Tear in of the muscle at the tendon-bone insertion in vivo ${ }^{7}$. 
The specific classifications of these tears vary. Furthermore, there are conflicts among the identification of the tear origin, tear propagation, and method of repair. A few studies report that the tears is expressed as interstitial (occurring in line with the tendon fibers), partial thickness, or full thickness. Partial-thickness tears occur more commonly ${ }^{2,6}$. The tear origin has been described as occurring in the anterior portion as it attaches into the lateral facet of the greater trochanter ${ }^{7,21}$. When the damage occurs, deep degeneration of the tendon progresses from a partial-thickness tear to full-thickness tear as it propagates posteriorly ${ }^{2}$. Generally, tears have a scarred band over the maximus or the medius (Figure 1.4). The full-thickness tears that results are noticeably large through MRI and ultrasound. This may cause greater trochanter pain syndrome (GTPS). The patients experience a dull, aching, lateral-sided pain that is frequently aggravated when weight-bearing and resisted hip abduction ${ }^{7}$. The GM tendon is recommended for examination in a manner similar to the rotator cuff. Furthermore, there are analogies to the shoulder in considering indications for debridement versus repair.

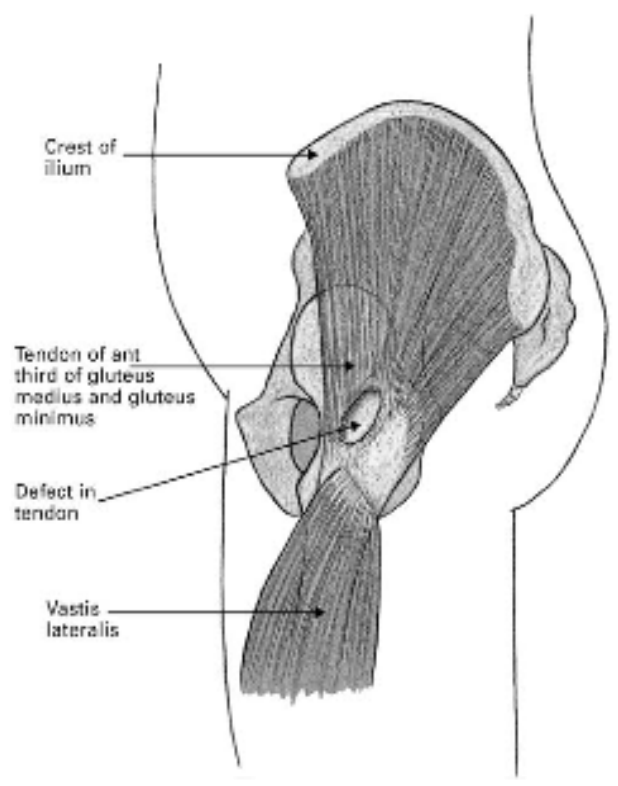

Figure 1.4- Oval shaped tear, where the underlying bone can be seen. The defect affects both the tendon and muscle . $^{8}$ 
Ultrasound can be used to evaluate the abductor tendons, identifying thickening and fluid consistent with tendinosis or the presence of partial or full thickness tears. MRI has also shown to be an effective diagnostic tool. This is commonly used when there is a suspected pathology of the gluteus medius and minimus tendons. An MRI can differentiate between partial and fullthickness tears by calcification at the tendon insertion and fatty atrophy within the muscle substance ${ }^{3}$.

\section{"Parallels" to the Shoulder}

Since GM tears have become more frequent, some have denoted this type of injury as the “rotator cuff" tear of the hip ${ }^{8}$. This description further adds knowledge to repair techniques; however increases the debate of how to classify this type of tear and how patients will be affected. In the hip, when a tear may be present, pain and weakness is shown during active, resisted abduction in extension and external rotation with the hip flexed to 90 degrees. Also, single leg stance lasting 30 seconds or longer can cause pain or discomfort ${ }^{3}$. Furthermore, when damage is present a tendinous defect occurs at the point of separation of the anterior and posterior flaps. In fact, the defect would appear in the form of a circular or oval shape (Figure 1.4) in the tendon. The bony surface of the greater trochanter is visible through the tear. It may extend in both anteroposterior and craniocaudal directions. The characteristics are usually rolled, mature edges ${ }^{9}$, and are often associated with the presence of free fluid in the trochanteric bursa. With the GM undergoing normal loading until treatment, this means the tear has been present and edges of the tear site are well defined and visible.

From these symptoms, some similarities are present between tears of the GM and rotator cuff. Tears in the shoulder affect supraspinatus and the anterior border of infraspinatus, where in the hip they involve gluteus minimus and medius. Rotator cuff tears in the shoulder demonstrate 
increasing incidence with age, as stated earlier in the hip. Furthermore, both will portray mature, rolled edges. GM and rotator cuff tears at the hip and the shoulder, respectively, cause weakness and are difficult to repair, and have similar radiological appearances ${ }^{8}$. When a rotator cuff is unrepaired, there often lead to fatty infiltration in the muscle, which is true for the GM.

Repairs of the rotator cuff are now being used or investigated for repairing GM tears. In fact, some clinicians evaluate, diagnose, and treat the gluteus medius in a similar fashion to the rotator cuff c $^{3,8,21}$. In cadaveric studies, traditional single row rotator cuff repairs have generally performed similarly with regard to biomechanical fixation. Additionally, failure of the aforementioned rotator cuff repair constructs tends to occur at the suture-tendon interface, which is a common clinical outcome ${ }^{15}$. Double Row techniques (Figure 1.5 and 1.6), however, have been found to be superior with respect to maximum load and shown smaller gap formation in relation to single-row techniques. In similar fashion to single row constructs, these repairs fail by tendon-suture rupture ${ }^{14}$. Unfortunately, biomechanical data for GM repairs is not as prevalent as for the rotator cuff because current research of hip related injuries are relatively new and clinical or anatomical information is investigated first for successful diagnosis.

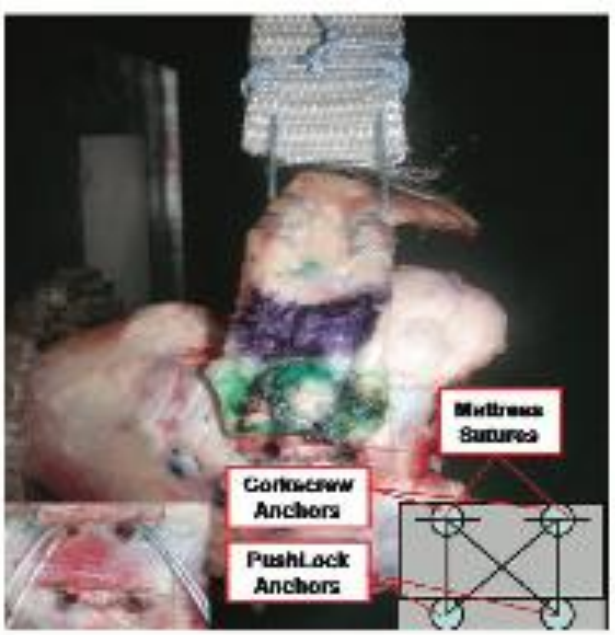

Figure 1.5- Rotator cuff repair using two kinds of anchors and a mattress stitch.

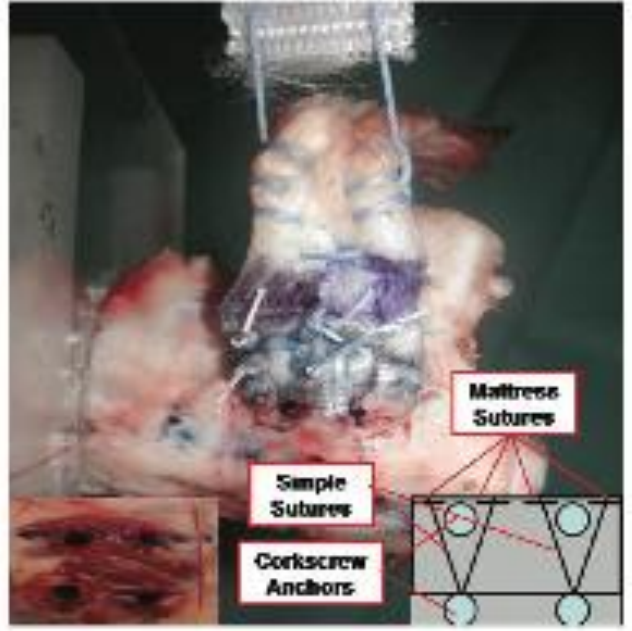

Figure 1.6- Rotator cuff repair using an anchor and two kinds of stitches: mattress and simple. 


\section{Surgical Intervention}

Treatments for GM tears vary with the severity of the pain and damage found. In some cases, patients receive symptomatic relief after physical therapy and steroid injections to the greater trochanteric bursa. At times, conservative management fails and there can be persistent symptoms lasting months to years ${ }^{2}$. Furthermore, there is an increased usage of MRI to detect previously unappreciated tear of the gluteus medius. These tears may go unnoticed in the past because diagnosing partial tears was difficult without MRI and further understanding of the anatomy. Recent advances in hip arthroscopy have led to significant innovations in the successful treatment of athletes and patients in the general public with respect to hip lesions ${ }^{7,21}$. In fact, surgeons have reported success with respect to repairing these tears ${ }^{10}$, but outcomes following repair are not well characterized by their healing process or biomechanically. . Controversially, many have described repairing as very challenging.

Arthroscopic and endoscopic techniques have been used to repair gluteus medius tendon tears with some clinical success. When a tear is present and disrupts the flaps, the anterior flap can be sutured to the posterior one, but this repair would be weak in its central portion. Usually, this type of repair is treated by three intraosseous sutures to provide direct reattachment ${ }^{8}$. When performing the repair arthroscopically, the similar difficulties lie within the attachment site. The attachment site of the tendon is usually prepared with a full-radius shaver similar to preparation of the footprint for rotator cuff tears (Figure 1.7). Depending on the size and shape of the tear, some of the tendon's area is lost. This, then, proves difficult to place suture anchors in the footprint ${ }^{7,21}$. Both metal and bioabsorbable anchors have been used depending on the bone quality (Figure 1.8). Based a clinical study ${ }^{3}$, patients had the presence of femoral neck fracture and the average age was 83.5 year old. Of these patients, $22 \%$ showed an incidence of tears not 
relating to the fracture. When repaired no anchors were used because of age, which could relate to bone quality. Although no bone mineral density was reported.

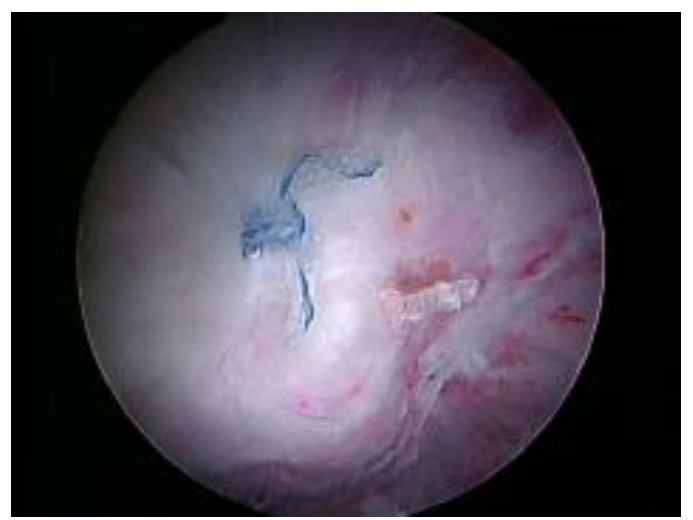

Figure 1.7- Arthroscopic image of suture anchors used to repair tendon in vivo ${ }^{7}$.

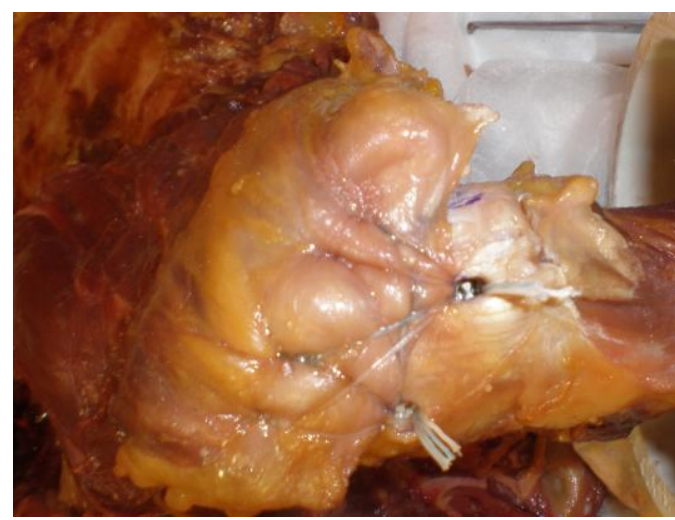

Figure 1.8- GM tendon sutured to the insertion site by anchors in vitro.

\section{Recent Gluteus Medius Findings}

Most, if not all of the existing research about the GM and the tears have been clinical or anatomical. The anatomical studies have begun to outline insertional footprint and basic dimensions of the GM along with other muscle on the greater trochanter. The clinical research has identified the actual tears, reported the onset of a tear, and described appropriate repair methods.

Anatomical research has produced information about the structure and functions of the GM. Investigators have begun to digitize the proximal femur and greater trochanter using a cadaveric model. These specimens were reconstructed by using 3-dimensional computer navigation software to quantify the footprints and muscles ${ }^{2}$ (Figure 1.9). Their findings provided the specific insertion site and area of the GM. The tendinous portion inserting into the superoposterior facet was found to be stout, with a shape of a circle with a radius of $8.5 \mathrm{~mm}$ (SD, $1.0 \mathrm{~mm})$. The surface area of this superoposterior insertion was reported as $196.5 \mathrm{~mm}^{2}(\mathrm{SD}, 48.4$ 
$\mathrm{mm}^{2}$ ). The tendinous portion attaching to the lateral facet was found to be more rectangular in shape (Figure 1.10). The surface area of this portion was $438.0 \mathrm{~mm}^{2}\left(\mathrm{SD}, 57.7 \mathrm{~mm}^{2}\right)$.

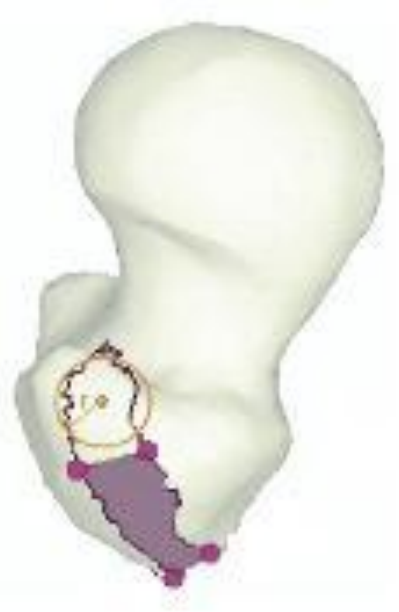

Figure 1.9- Reconstructed GM footprint. Shaded area is the anterior section ${ }^{2}$.

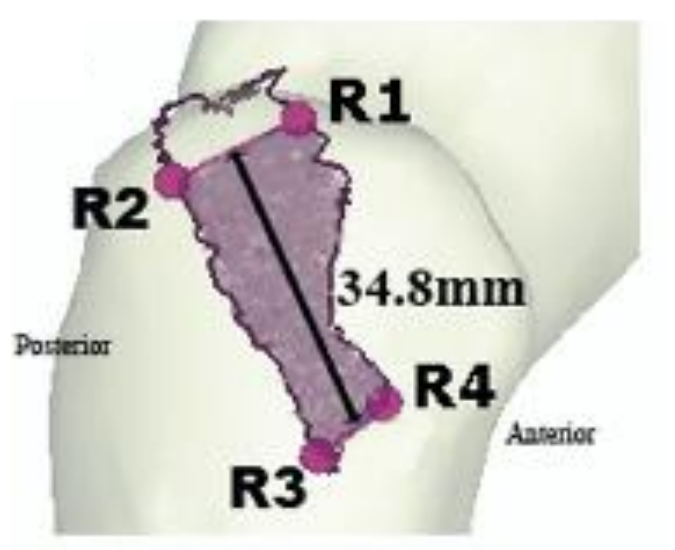

Figure 1.10- Footprint given points for measurements. Length of anterior footprint based on furthest points ${ }^{2}$.

From these values and what is known clinically, it was concluded that the superoposterior portion is thought to stabilize the femoral head in the acetabulum during heel strike. The lateral portion of the GM inserts broadly onto the lateral facet of the greater trochanter. It accounts for the vast majority of the lateral facet footprint. In addition to aiding in abduction, the lateral origin of these fibers allows it to function as an external rotator of the pelvis to allow for swing-through of the lower limbs.

Clinical studies have developed the use of MRI in order to diagnose tears and use advancements in imaging to find damage before it worsens. In recent experiments, surgeons have used patients with lateral-side hip pain and performed a series of tests to determine how the damage alters gait and posture ${ }^{7}$. These patients were evaluated prospectively and followed-up after 25 months (range, 19-38 months). Preoperative, intraoperative, and postoperative data were analyzed. Diagnosis was made by physical examination and magnetic resonance (Figure 1.11) imaging and was confirmed at the time of endoscopy in all cases. 


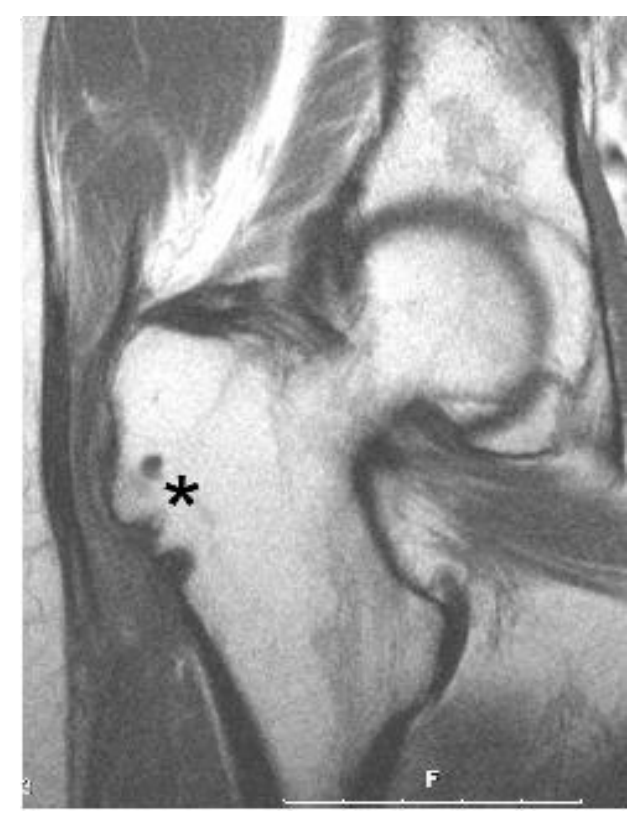

Figure 1.11- Patient based MRI images after successful GM repair. Asterisk denoted anchor position? ${ }^{7}$.

The postoperative rehabilitation consisted of 6 weeks of crutch-protected weightbearing with the goal of $20 \mathrm{lb}$ of pressure on the operative extremity. At 6 weeks, active range of motion was begun, and early abductor strengthening was started. By the third month, strengthening continued and sport-specific activity was begun by month 4 . All 10 cases experienced complete resolution of lateral hip pain, and $100 \%$ of patients regained full motor strength ${ }^{21}$.

\section{Tendon}

Tendon is the dense fibrous connective tissue between muscle and bone, and its main function is to transfer load generated by muscle to $\operatorname{limb} b^{12}$. Most of the tendon is composed of extracellular matrix (ECM). Tendon ECM contains a predominant amount of the fibrous protein type I collagen (70-80\% of dry weight) together with a small amount of elastin (1-2\% of dry weight), and a ground substance of glycoproteins, proteoglycans, and water ${ }^{13}$. Tendon collagen is highly organized and arranged as fibrils, fibers, and finally fascicles (Figure 1.12), which pack 
together to form the tendon unit ${ }^{12}$.

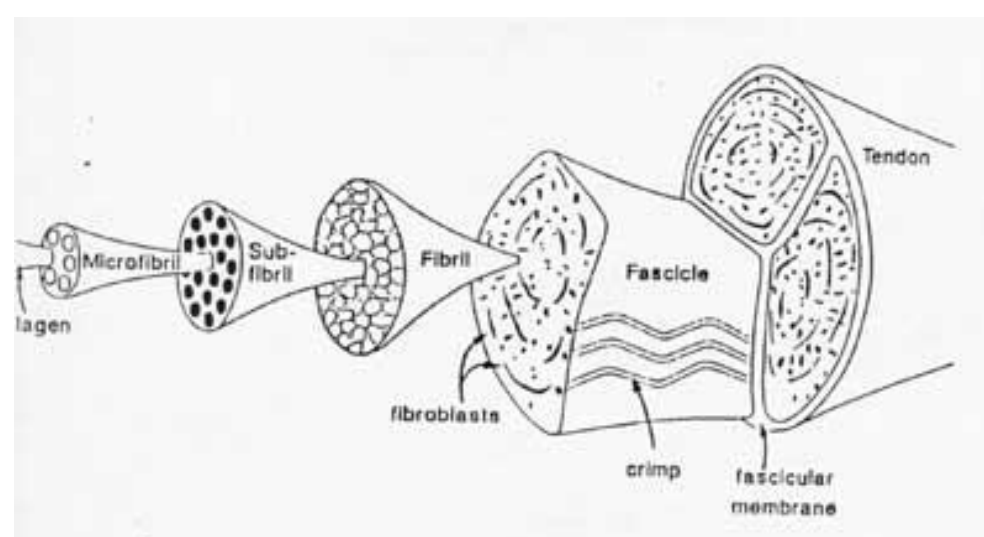

Figure 1.12- Structure hierarchy of tendon. (http://www.engin.umich.edu/class/bme456/ligten/ligten.htm)

Tendon collagen structure has mechanical and viscoelastic properties. Initially collagen fibrils are crimped in 0-2\% strain which is considered the toe region. As the tendon elongates, fiber crimp reduces and the bundles align in parallel. When the deformation continues into what is known as the plastic region, mechanical properties are compromised (Figure 1.13). When the tendon has undergone plastic deformation, the fibers have reached irreversible deformation without any increase in stresses or loads. Finally, the tendon ruptures as it reaches the failure region. 


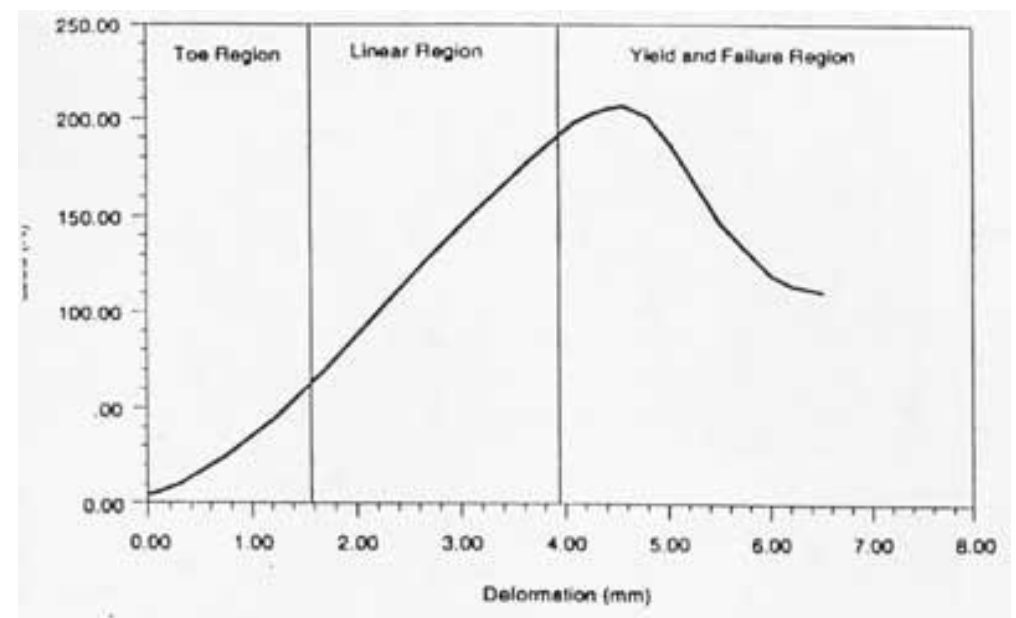

Figure 1.13- Mechanical response of the tendon. (http://www.engin.umich.edu/class/bme456/ligten/ligten.htm)

Viscoelastic property is known as creep and stress relaxation performance (Figure 1.14). Both are time dependent mechanical behavior. They are not constant, but depend on the time of displacement or load. Stress relaxation means that stresses decrease if they are pulled to constant deformations. Creep means deformation increase under a constant force. Tendon has the ability to adapt to different loading conditions and physiologic lengths without failure. Another viscoelastic property is hysteresis or energy dissipation. This means that a material is loaded and unloaded; the unloading curve will not follow the same path as the loading curve. The difference between the two curves represents the amount of energy that is dissipated or lost during loading. After numerous cycles, the tendon will approach a mechanical steady state. This is demonstrated by the load or displacement going unchanged during the last few cycles. 

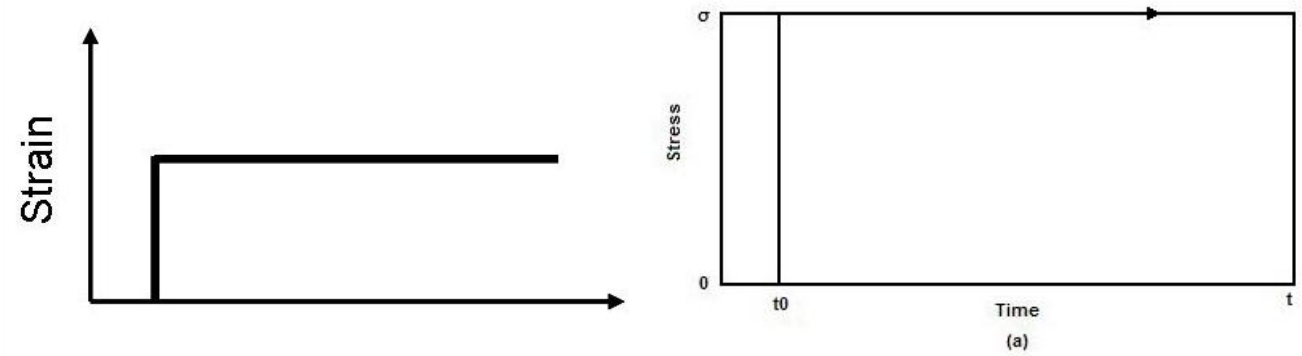

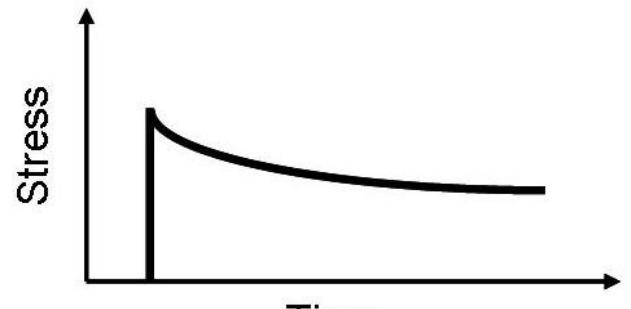

Time

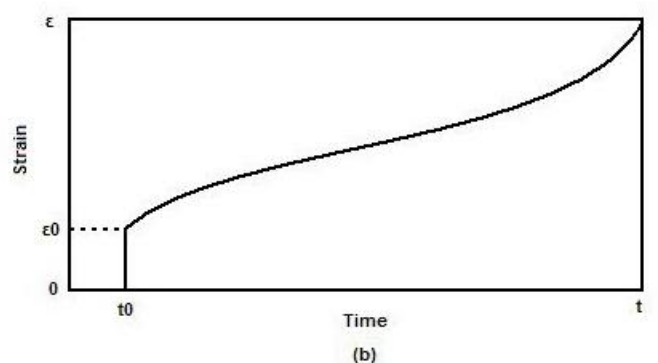

Figure 1.14- Viscoelastic phenomenons in tendon ${ }^{12}$

In order to better understand the tendon properties, elongation, deformation, etc can be evaluated by crosshead-based displacement from a testing system or from optically based measured deformation/strain. Both do not have the ability to assess microscopic deformation. Though, optical measurements can give insight to local strain and how the muscle or tendon reacts to loading in a certain area. The more references that are used, the better the variation across the tissue will be detected. Crosshead-based displacement will indicate whole structure properties, instead of regional properties.

\section{Motivation and Objective}

Successful repairs of the GM have been reported and depend on bone quality, repair type, and severity of tear. While repairing the tear is expected to relieve pain and restore normal movement of the pelvis, little is known about the mechanical properties of the repair and muscle. Furthermore, the tear itself causes pain and weakness, which affects gait and muscle function. 
There is no existing study that investigates the mechanics of the intact GM. A more complete understanding of the anatomy, native, and repaired GM may help facilitate surgical planning and rehabilitation. Therefore, the present investigation evaluated mechanical properties of the intact and repaired GM tendon.

Specific Aim 1: Characterize the mechanical properties of the intact GM muscle-tendon-bone unit.

Specific Aim 2: Compare the biomechanical performance of two selected repair techniques, Double Row with Massive Cuff Stitch or Double Row with Knotless Lateral Anchors, for a complete GM tendon tear. 


\section{Section 2: Methods}

\section{CT Scan}

Sixteen fresh-frozen human cadaveric hemi-pelves ( 8 contralateral pairs) from 7 females and 1 male were obtained. There was no evidence of any bone fracture or soft tissue damage. The mean age of the donors was $55 \pm 3$ years old (Range: $35-63$ ). Before any specimens were dissected, each hemi-pelvis was CT scanned (Figure 2.1) in anatomical neutral position, where the head of the patient enters the scanner first (BrightSpeed, GE Medical Systems, Fairfield, CT). The specimens were scanned ( $3 \mathrm{~mm}$ slices, $0.5 \mathrm{~mm}$ pixel size, $120 \mathrm{KV}$ ) in order to evaluate the bone mineral density (BMD). By using Mimics software (Materialise 13.1, Luven, Belgium), the greater trochanter was viewed in the axial direction. A box of similar area (Avg: $30.44 \pm$ $0.22 \mathrm{~mm}^{2}$ ) for all specimens was made over the repair site on the greater trochanter, and only trabecular bone was included because any deterioration would occur in this type of bone. A first bone density was taken from the slice where the greater trochanter is fully visible and the outlined cortical bone and the trabecular bone can be distinguished. Then, by scrolling up one slice in the cranial direction, another bone density was measured. The same procedure was performed for a third slice in the cranial direction, so three total bone densities were measured for one specimen (Appendix). The densities were averaged across the three slices and reported as Hounsfield units, a grayscale value.

A phantom was scanned with the specimens and the same phantom was used for each scan session. This was used to measure pure trabecular bone, in which different colored, circular plugs are used to depict dense bone, pure cortical bone, and air. Measurements were made in a similar fashion as stated before. The phantom values were used to find the offset of the specimen 
scanned from the "healthy" bone. The purpose was to see how close the specimens obtained were to healthy bone and to help in the randomization process.

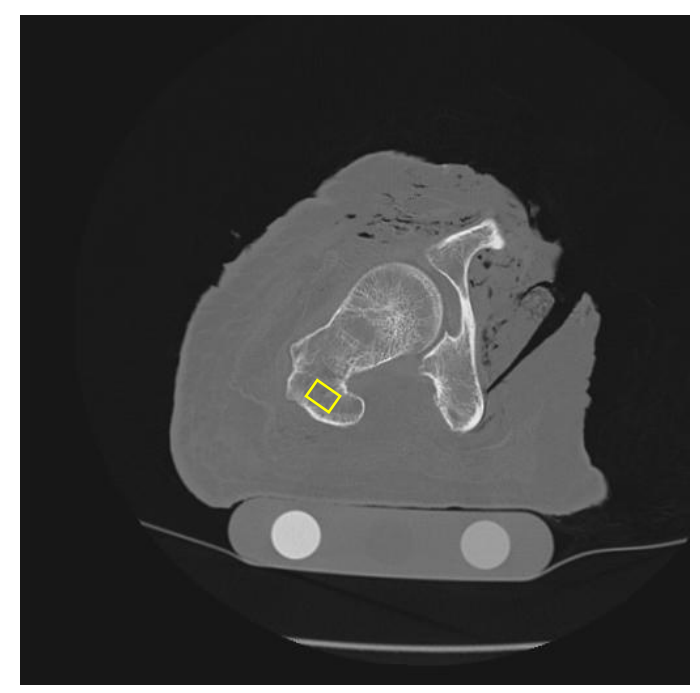

Figure 2.1- CT image of greater trochanter in the coronal plane. Box demonstrates the area in which the density was measured.

\section{Specimen Preparation}

From BMD calculations, the specimens were randomized and assigned to one of three test groups of similar mean BMD to rule out its affect on the results. The three groups included Intact ( $n=4)$, Double Row with Massive Cuff Stitch (DR-MCS) repair (n=6), and Double Row with Knotless Lateral Anchors (DR-KLA) repair $(n=6)$. During specimen randomization (Table 2.1), a given contralateral pair was not assigned to the same group. Furthermore, the Intact specimens showed no evidence of tears or damage to the muscle. 
Table 2.1- Specimen details and groupings.

\begin{tabular}{|cccccc|}
\hline Specimen ID & Left/Right & Gender & Age & BMD & Group \\
\hline BRC 0908026 & L & F & 55 & 445.5 & Intact \\
\hline BRC 0904016 & L & F & 57 & 531.3 & Intact \\
\hline BRC 0911027 & R & F & 57 & 780.6 & Intact \\
\hline BRC 1005006 & R & F & 59 & 754 & Intact \\
\hline BRC 0908026 & R & F & 55 & 758.9 & DR-MCS \\
\hline BRCIL 0110001 & L & F & 57 & 231.5 & DR-MCS \\
\hline BRC 0807015 & R & F & 57 & 464.7 & DR-MCS \\
\hline BRC 0912022 & L & F & 63 & 426.9 & DR-MCS \\
\hline BRC 0911027 & L & F & 57 & 752.8 & DR-MCS \\
\hline LWTF 09011 & R & M & 35 & 918.2 & DR-MCS \\
\hline BRC 1005006 & L & F & 59 & 784.7 & DR-KLA \\
\hline BRCIL 0110001 & R & F & 57 & 256.3 & DR-KLA \\
\hline BRC 0807015 & L & F & 57 & 383.9 & DR-KLA \\
\hline BRC 0904016 & R & F & 57 & 566.6 & DR-KLA \\
\hline BRC 0912022 & R & F & 63 & 433.3 & DR-KLA \\
\hline LWTF 09011 & L & M & 35 & 925.8 & DR-KLA \\
\hline
\end{tabular}

Each hemi-pelvis was dissected such that only the gluteus medius tendon-muscle unit and joint capsule were preserved. The femur was cut transversely using a bone saw, leaving about 130mm distal from the greater trochanter intact. The femur was then potted within PVC piping (76mm outer diameter) using acrylic cement (Isocryl, Lang Dental, Wheeling, IL). The gluteus medius muscle was cut (Figure 2.2) to not exceed 50mm wide or 130mm long (proximal-distal) in order to secure the muscle belly within a custom cryo-clamp. 


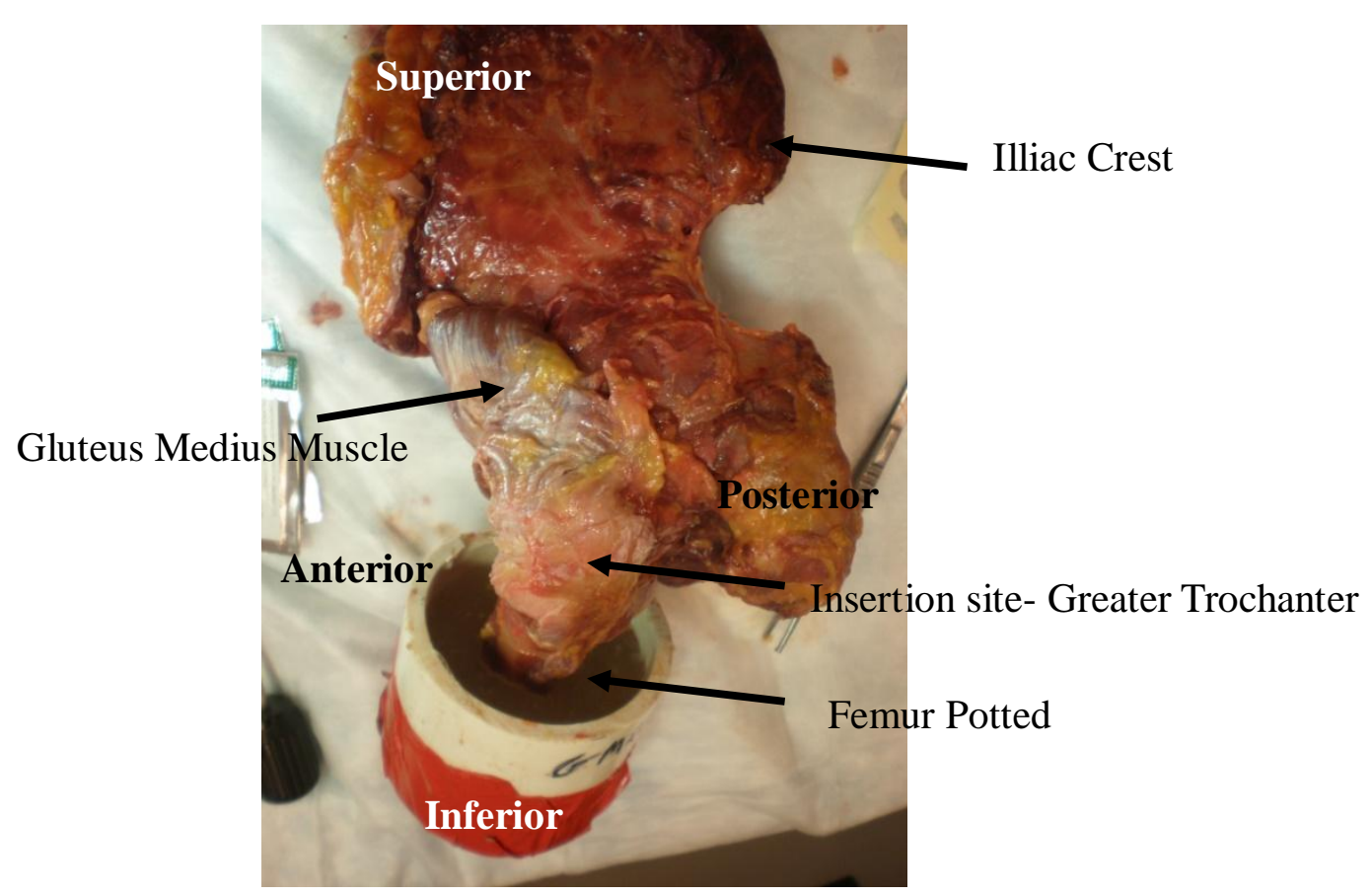

Figure 2.2- Anatomical landmarks of the hip joint. Major constructs are labeled and femur was potted in PVC. Hip capsule is deep to the insertion site.

For the 12 specimens receiving repair constructs, the gluteus medius tendon was sharply released from its anatomic footprint (Figure 2.3) using a \#15 blade. Footprint lengths (lateral, superoposterior, and total) were marked using a skin pen and measured using a digital caliper for comparison between repair groups (Figure 2.4). One footprint site is located on the superoposterior facet of the greater trochanter with its orientation in line with the femoral head. The other site is located on the lateral facet and accounts for a vast majority of the footprint ${ }^{2}$. 


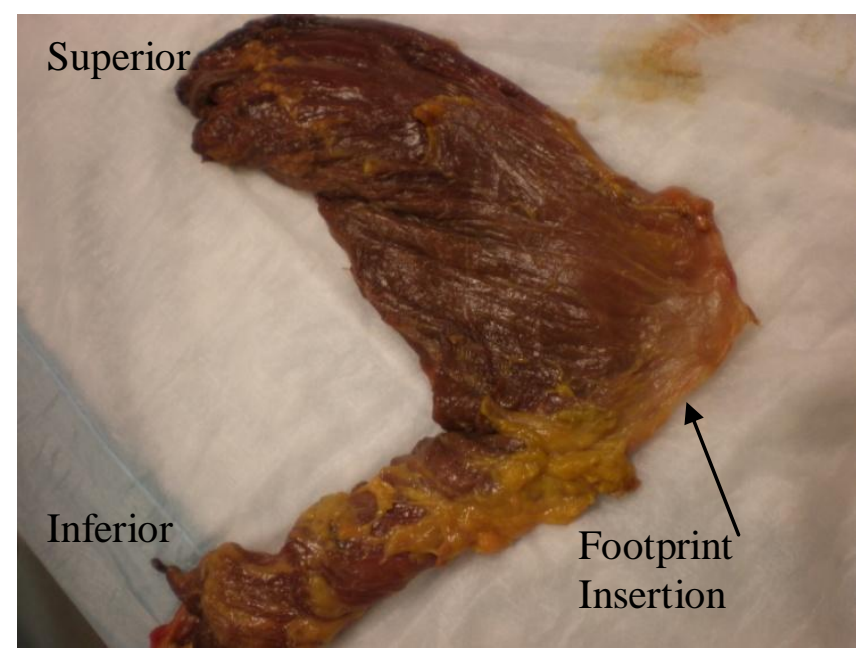

Figure 2.3- Entire GM muscle-tendon excised at insertion point.

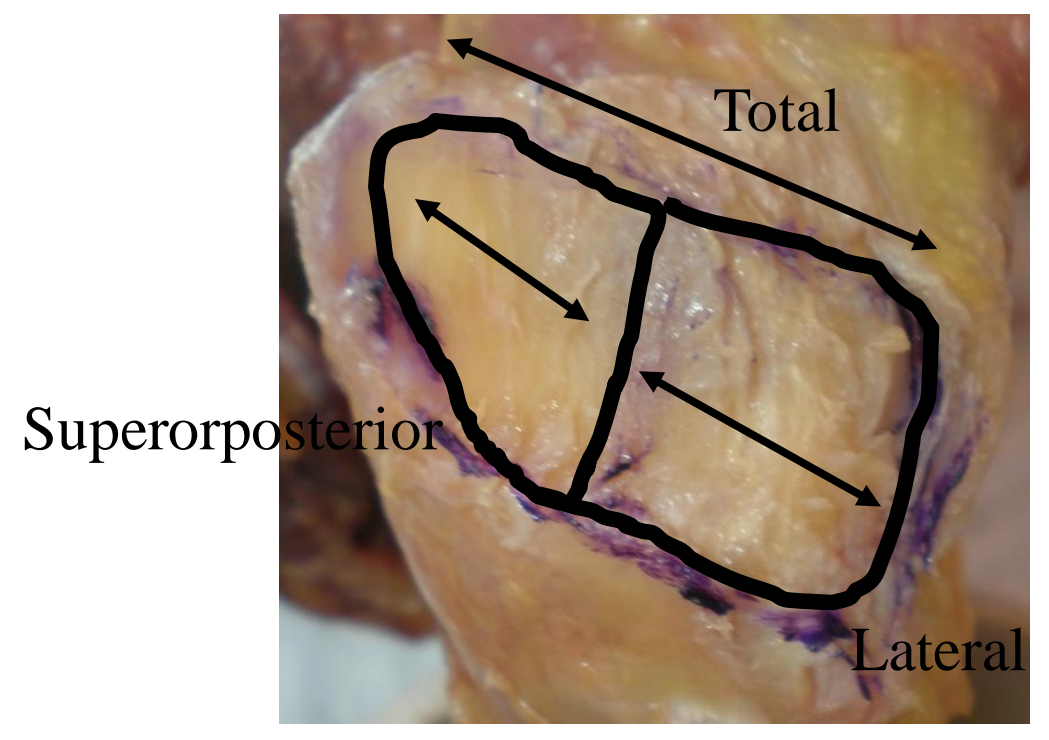

Figure 2.4- Outlined Lateral and Superoposterior footprint.

The Double Row with massive cuff stitch (DR-MCS) repair used two medial row, doubleloaded suture anchors (5.5 TwinFix, Smith \& Nephew, Andover, MA) with four mattress stitches (Figure 2.6) and two lateral row, double-loaded suture anchors with four simple stitches ${ }^{14}$. The simple stitches from the lateral anchor were placed medial to the mattress stitches (Figure 2.7) from the medial anchors to create a massive cuff stitch configuration ${ }^{15,16}$. The Double Row with 
Knotless Lateral Anchors (DR-KLA) repair used two medial row, double-loaded suture anchors with four mattress stitches and two lateral row, Knotless anchors (Figures 2.8 and 2.9) (5.5 Footprint PK, Smith \& Nephew, Andover, MA). Intact specimens did not show evidence of tears or pathology of the gluteus medius tendon or evidence of bony fractures.

(a)

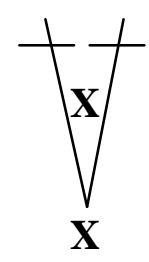

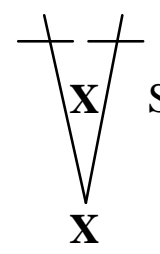

Suture Anchors

(b)

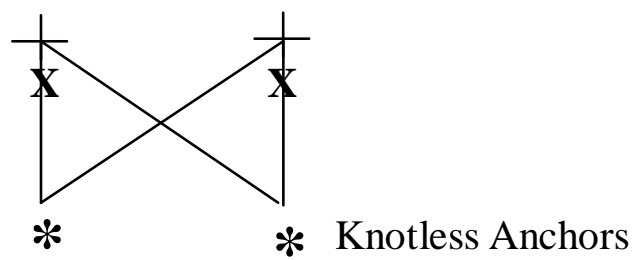

Figure 2.5- Schematic of (a) DR-MCS and (b) DR-KLA. Different anchors are marked.

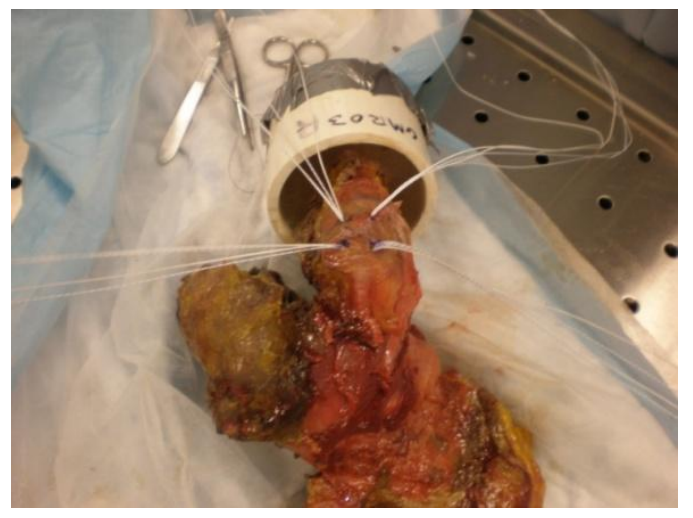

Figure 2.6- DR-MCS repair. Suture anchors drilled into the greater trochanter.

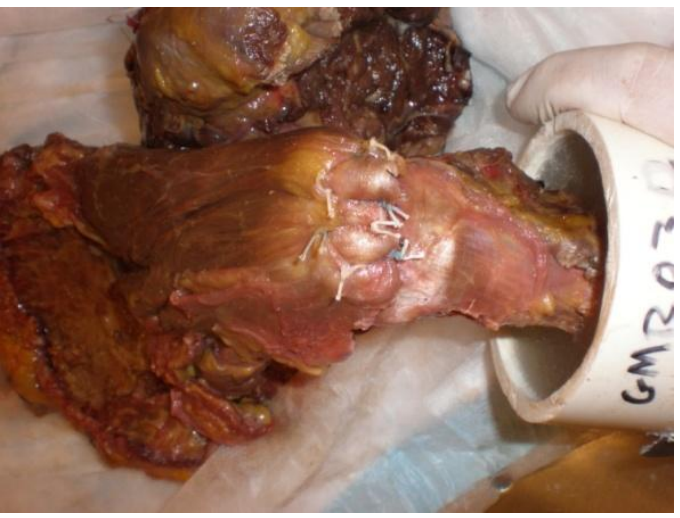

Figure 2.7- Mattress and simple stitches are used. Sutures are placed through the tendon and tied down the bone. 


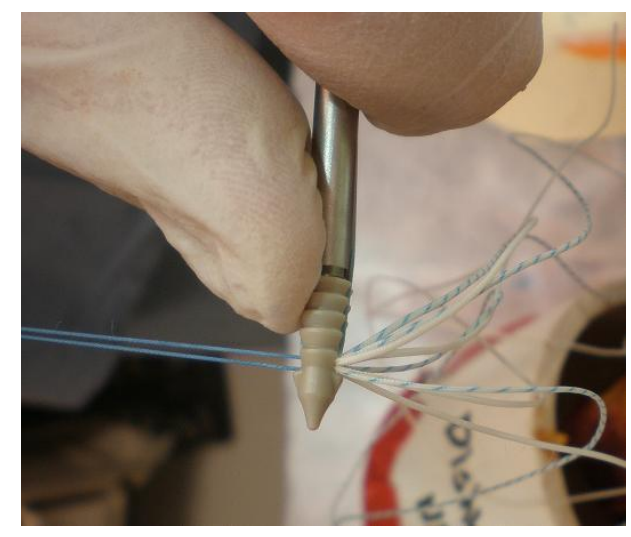

Figure 2.8- DR-KLA repair. Two Knotless anchors are used.

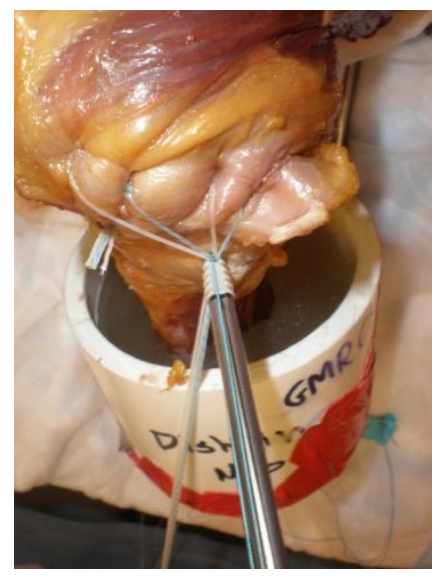

Figure 2.9- Knotless anchors with sutures placed into the bone just below tendon. All sutures are tightly connected to anchor.

\section{Biomechanical Testing}

The potted femur was placed into a custom alignment apparatus to facilitate positioning of the gluteus medius muscle in a neutral anatomical stance (muscle at $20^{\circ}$ lateral). The muscle was secured within a custom cryogenic clamp ${ }^{17}$ fixed (Avg gage length: $41.4 \pm 2.4 \mathrm{~mm}$ ) to the actuator of a materials testing machine (Insight 5, MTS Corporation, Eden Prairie, MN). Dry ice was allowed about 10 min to thoroughly freeze the muscle to the clamp at about $0^{\circ} \mathrm{C}$. It was left in throughout testing to ensure the muscle never defrosted and new ice was placed after the cyclic test. The temperature was maintained during the transition from the cyclic test to the pull to failure test. Throughout testing, the muscle, musculotendinous junction, and proximal tendon temperatures were maintained at a minimum temperature of $19^{\circ} \mathrm{C}$ (as verified using an infrared thermometer) by using a warm saline spray.

Black, circular markers ( $3.3 \mathrm{~mm}$ in diameter) were glued to the tissue surfaces in order to track their displacements optically. The configuration of the markers for the repair groups (Figure 2.10) consisted of a (medial-lateral) row of three markers on the bone, a row of three markers on the muscle, and separate rows of two markers closest to the repair site (on bone) and 
most proximal to the sutures (on muscle-tendon). Marker placement for intact specimens (Figure 2.11) consisted of two rows of three markers each on the tendon, a row of two markers on the bone, and a row of two markers near the lower edge of the cryo-clamp. Smaller markers (2.4 and $1.6 \mathrm{~mm})$ were investigated during pilot testing, but were not visible by the tracking system. Furthermore, a different marker configuration was tested, in which more marker were placed around repair site (both on the bone and tendon). But, the current placement was optimal due to muscle size, distinguishing between markers, investigating movement of anchors, and capturing movement across the muscle. Though for the Intact group, because care was taken to the tendon, the insertion site could not be identified as well as the repair group. The possibility of cutting the GM tendon and the overlapping muscles near the GM muscle made pointing out the insertion site difficult. Therefore, the markers were placed as close to the insertion as possible and had no restrictions on placement since there were no sutures or anchors were present.

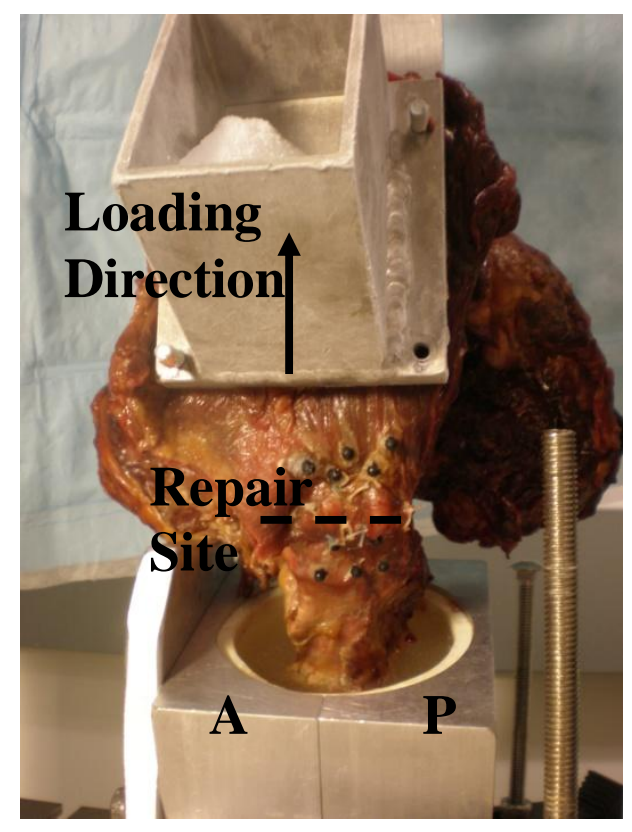

Figure 2.10- Repair configuration where markers are aligned to evaluate segments.

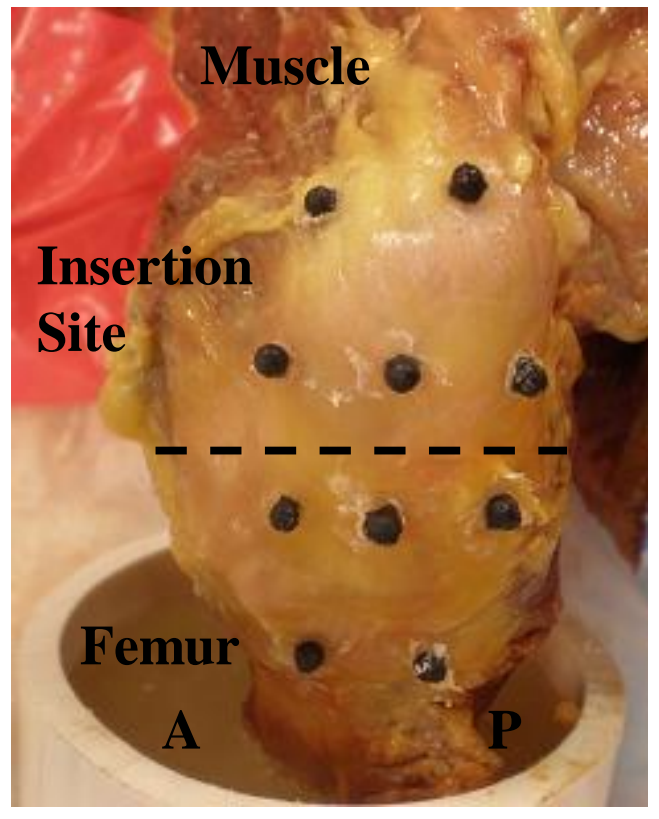

Figure 2.11- Intact configuration where markers are placed to capture changes around tendon insertion. 
The intact specimens were subjected to a $10 \mathrm{~N}$ preload for 2 minutes followed by a load to failure test at $1 \mathrm{~mm} / \mathrm{sec}$. Results from these specimens were used to establish the cyclic protocol for evaluating the two repair constructs; specifically, a peak cyclic load of $10 \%$ of the mean intact failure load was selected as it corresponded to the lower linear region of the loaddisplacement curve (Figure 2.12). The repaired specimens (Figure 2.13) were subjected to the following testing sequence: (1) $10 \mathrm{~N}$ preload for 2 minutes, (2) 150 cycles from $10 \mathrm{~N}$ to $125 \mathrm{~N}$ at $90 \mathrm{~N} / \mathrm{s}$, and (3) load to failure at $1 \mathrm{~mm} / \mathrm{sec}$. Throughout testing, digital video was acquired at 48 $\mathrm{Hz}$ (synchronized with time, crosshead displacement, and load from the MTS output) for subsequent optical video capture (Digital Motion Analysis System, Spica Technology Corporation) comprised of a high-resolution digital video camera (Imperx IPX-1M48-L, 1000x1000x48 fps, Boca Raton, FL) of the markers ${ }^{18,19}$. Calibration studies conducted in our laboratory revealed that the precision of the measurement system is about $2.3 \mu \mathrm{m}$. The setup replicated the testing setup of the specimens.

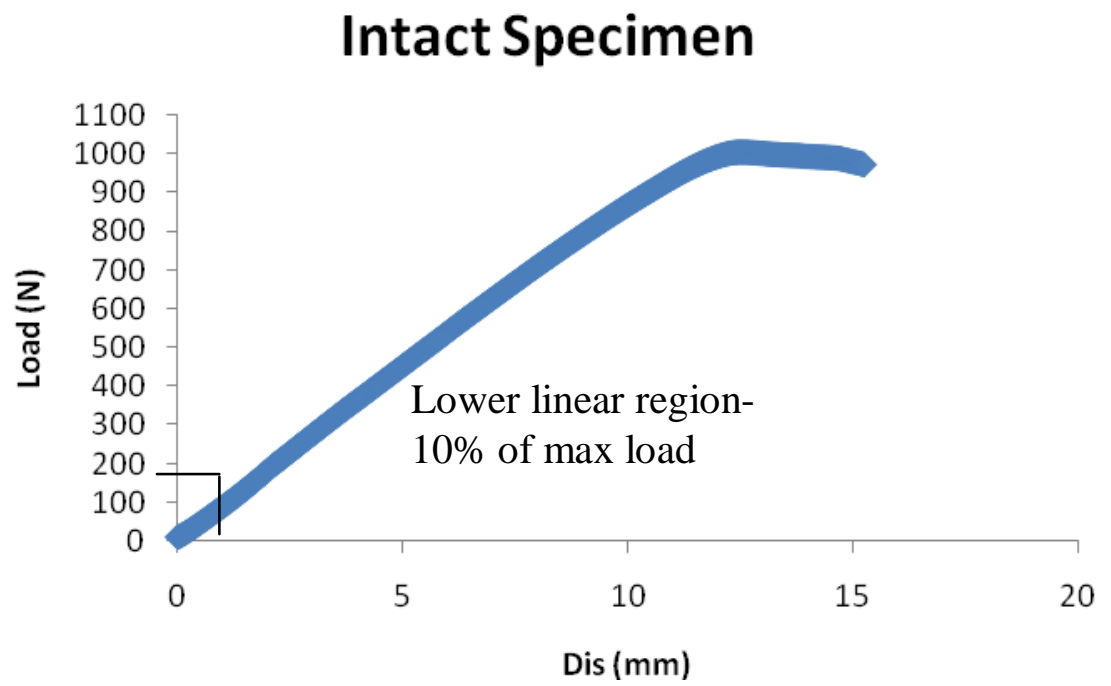

Figure 2.12- Intact load vs displacement curve from MTS. Lower linear region values were selected for cyclic loads for the repair groups. 


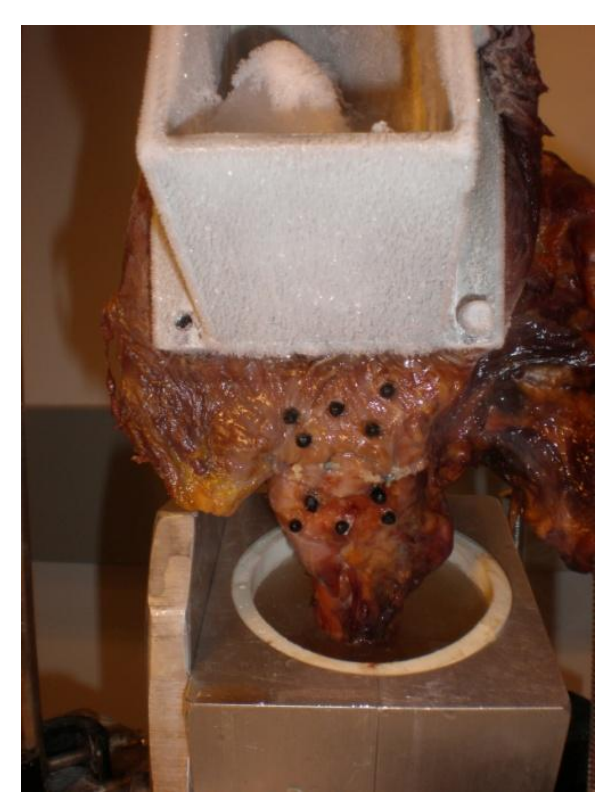

Figure 2.13- MTS setup. Muscle is frozen to clamp with dry ice.

\section{Data Analysis}

Due to the small sample size $(n=4)$, failure data for intact specimens were computed but not statistically compared to those of the repair groups. Mechanical data for the Intact group was obtained from the MTS and consisted of maximum load, stiffness (steepest slope of loaddisplacement curve spanning $30 \%$ of data points from test initiation to failure point ${ }^{20)}$, displacement at max load, and failure mode. For the repair groups, biomechanical data from the cyclic test included cyclic elongation, elongation amplitude, and secant stiffness (Figure 2.14). Cyclic elongation was defined as the net crosshead displacement from the peak of the first cycle to the peak of the last cycle. Elongation amplitude was calculated as the peak-to-valley crosshead displacement for the final test cycle ${ }^{20}$. Secant stiffness was considered the slope of the line joining the minimum and maximum points of the loading phase of the force-deformation curve.

Load to failure test outcomes included maximum load, yield load, normalized yield (with respect to max load), stiffness (steepest slope of load-displacement curve spanning $30 \%$ of data 
points from test initiation to failure point ${ }^{20}$ ), post-yield crosshead (Figure 2.15) extension (extension from yield load to maximum load), normalized work to yield (with respect to work to maximum load), and failure mode. Yield load was found by the MTS using an offset value.

Specifically, the load at the offset yield is defined by a data point that is the intersection of the curve by the X (displacement) and Y (load) channels and the line specified by the slope. The line is shifted in the $\mathrm{x}$-direction by the amount of the offset value. Thus, this value is an integer, but corresponds to the yield load at this point.

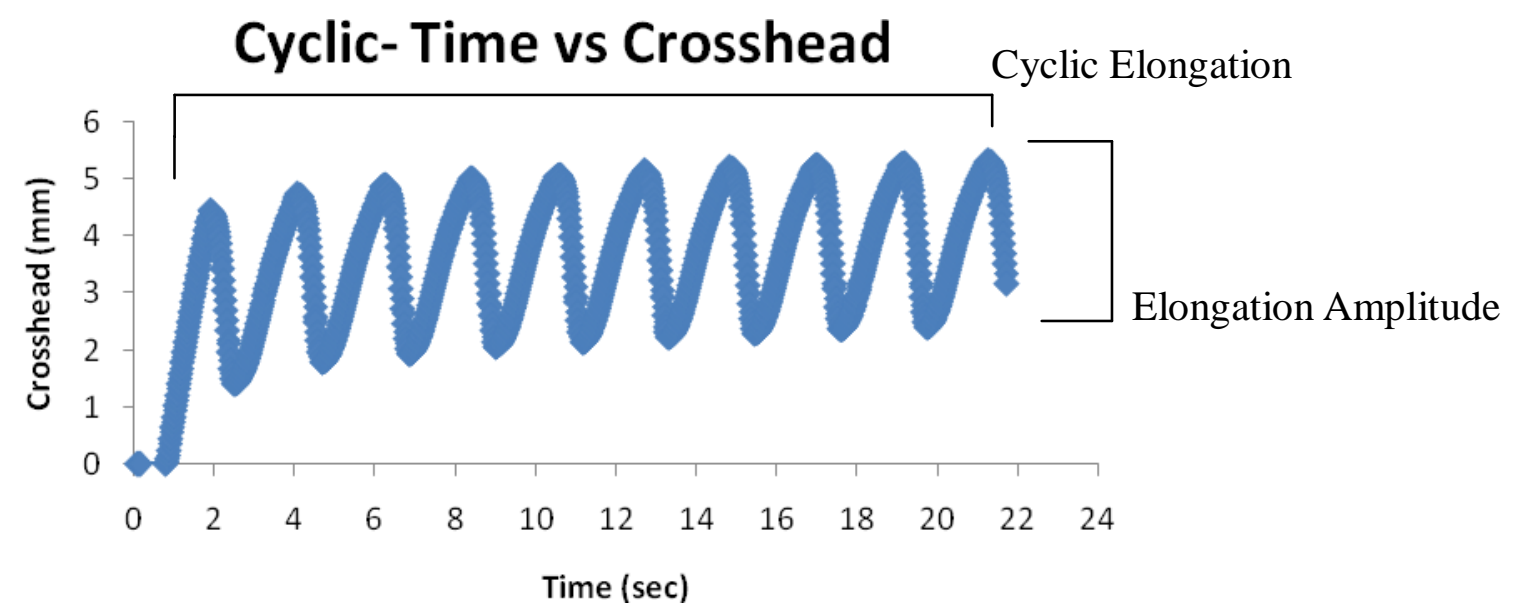

Figure 2.14- Representative results from cyclic testing of repaired construct. Cyclic elongation and elongation amplitude parameters are illustrated. 


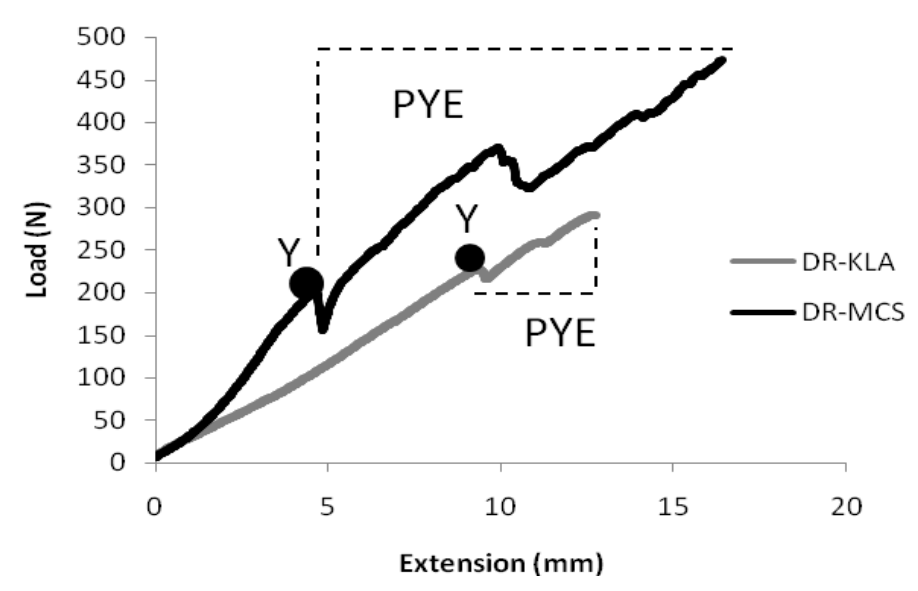

Figure 2.15- Load to failure test curve from MTS. Typical outcome for each repair. $\mathrm{Y}=$ yield load. $\mathrm{PYE}=$ post yield extension, extension from yield load to max load.

Optical marker data were analyzed by ImageJ software (National Institute of Health (NIH), Maryland, USA) using the MTrack2 plug-in (valelab.ucsf.edu/ nico/ijplugins/MTrack2.html). Full explanation and figures can be found in the Appendix. By using a screenshot of the calibration frame from the DMAS, distances between crosshairs on the calibration were measured in ImageJ (in pixels) for predetermined lengths (in $\mathrm{mm}$ ) and conversion ratio from pixels to millimeters was determined. Following image calibration, centroids (i.e., $x-y$ coordinates) of each marker were tracked for all frames of the failure test. An optical segment length was computed from a pair of surface markers as the shortest distance between the respective markers ${ }^{20}$. For each frame of the tracked video, the percentage change in segment length with respect to that of the preloaded state was defined as the relative segment elongation (RSE); this index was computed for segments connecting the muscle and bone (MB) markers as well as those spanning the repaired tendon unit (RTU) (Figures 2.16). Furthermore, both were calculated for the lateral and superoposterior aspects of the constructs. Optical results computed included RSE at yield load, maximum load, and relative post yield segment elongation. RSE of 
the MB and RTU were calculated at yield and maximum load for the lateral and superoposterior side.

Optical data for the Intact group was analyzed using the same technology, and two different ways because of the marker configuration. The coordinates were obtained from MTrack2 (x,y) and the conversion ratio was determined. The data was evaluated by selecting the markers that were in line with each other and averaging the $x-y$ coordinates. Thus, the tissue was divided into separate rows (Figure 2.17), which included Lower Trochanter (closest to the potted femur), Below Insertion (insertion site), Above Insertion (insertion site), and Tendon-Muscle (tendon-muscle junction). The distance of the averaged coordinates were calculated for each row. The data was conveyed in millimeters and compared among the different rows.

Furthermore, the same optical protocol for the relative segment elongation (RSE) was used for Intact group. This index was computed for the segments spanning the tendon to bone and near the insertion site on the superiorposterior and lateral side. The data was analyzed at maximum load to distinguish any variation in segment elongation between sides during loading. Thus, only three specimens were used because one failed at slightly above $400 \mathrm{~N}$ and failed differently than seen clinically. 


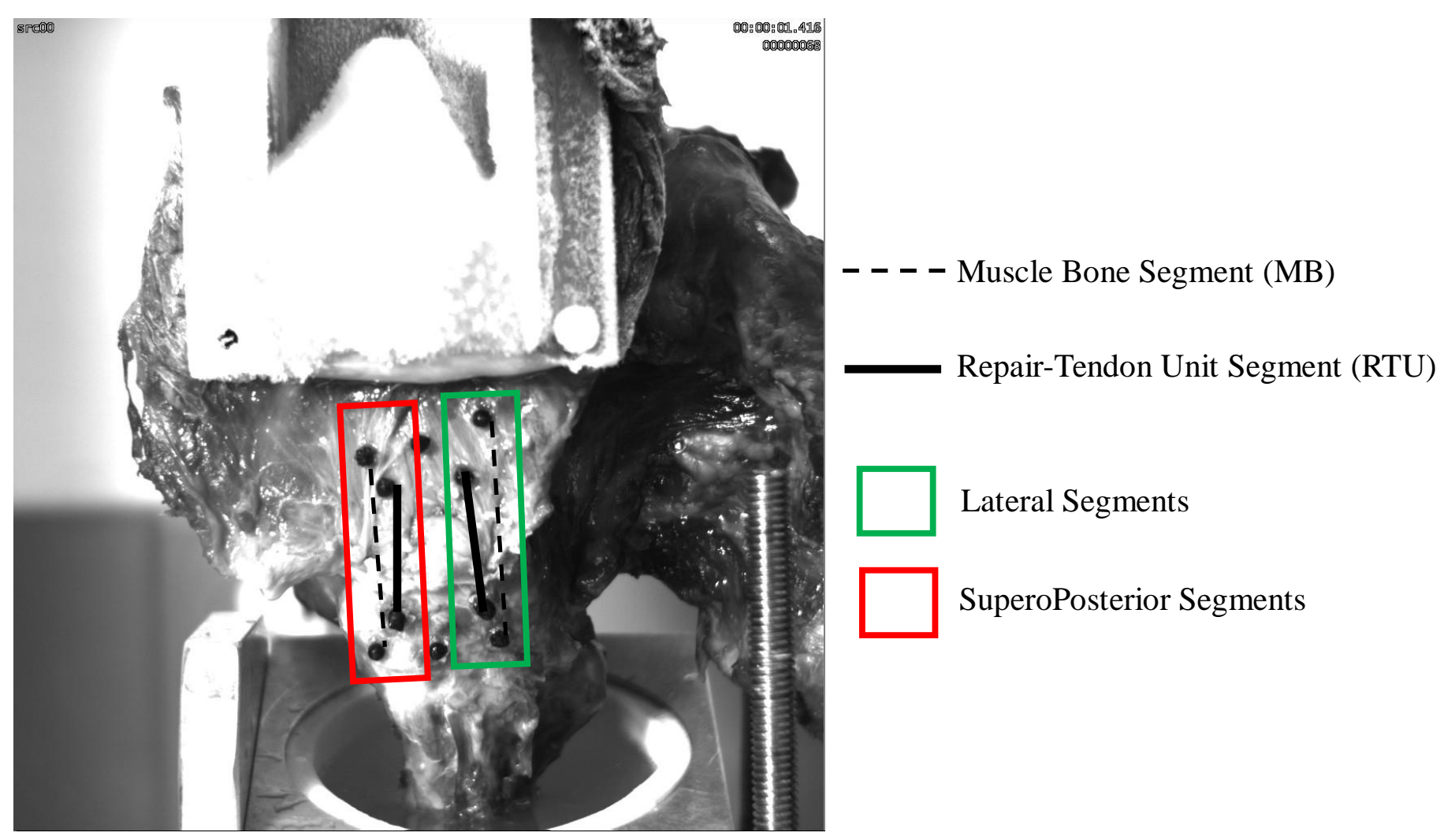

Figure 2.16- Division of markers for analysis, in which the PSL data is obtained for specific areas of the tissue. Key demonstrated the segments and regions that are being analyzed.

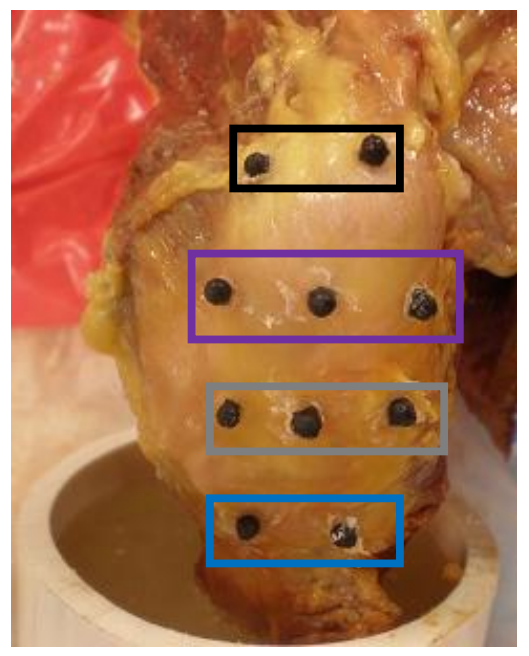

\section{Tendon-Muscle}

Above Insertion

Below Insertion

Lower Trochanter

Figure 2.17- Marker placement and defined regions for optical analyses. In the different regions, rows were averaged and analyzed from the preloaded state to max load. 
Furthermore, the data from the repair cyclic and failure tests underwent linear regression analysis. Linear regression analyzes the relationship between two variables. For each test, both X and $\mathrm{Y}$ values are known, in which the $\mathrm{X}$ value corresponds to displacement and $\mathrm{Y}$ value to load (both from the MTS). The purpose is to find the best straight line through the data. In the current study, the slope has a scientific meaning, in which the linear region slope corresponds to the stiffness of a specimen.

In MatLab, a previously established code (Appendix) was used, where the input correlates to the $\mathrm{X}$ and $\mathrm{Y}$ values. The input is displayed as the curves for each of the specimens from the preloaded state to yield load (Figure 2.18). The code then performs a linear regression of selected points. The output displays a red line over the original curve as well as three values: toe region length, slope of the toe region, and slope of the linear region. The two slopes are distinguishable (Figure 2.19). Also, an $\mathrm{R}^{2}$ value is given to see how well the line fit over the original curve. The toe region slope is considered the nonlinear section at the beginning of the test, where the tendon fibers are unraveled and beginning to organize. The linear region is considered the part of the curve where the fibers are tense and stretching. The toe region length is considered the distance of the first best fit line over the toe region to the second best fit line (linear).

Data points up to yield load were selected because the repair may be compromised after. Thus, the stiffness may change and deviate from the best fit line when incorporating data to maximum load. To verify the accuracy of the best fit lines, the minimum $\mathrm{R}^{2}$ value for the toe region and linear region was 0.84 . 


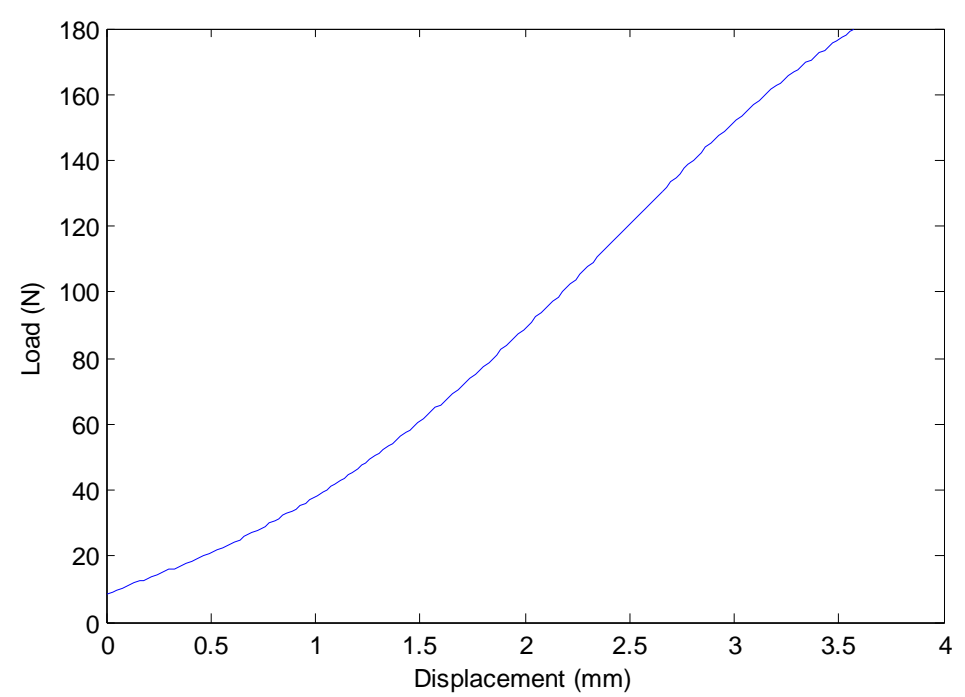

Figure 2.18- Original curve of specimen from MatLab. Data is from preload to yield load.

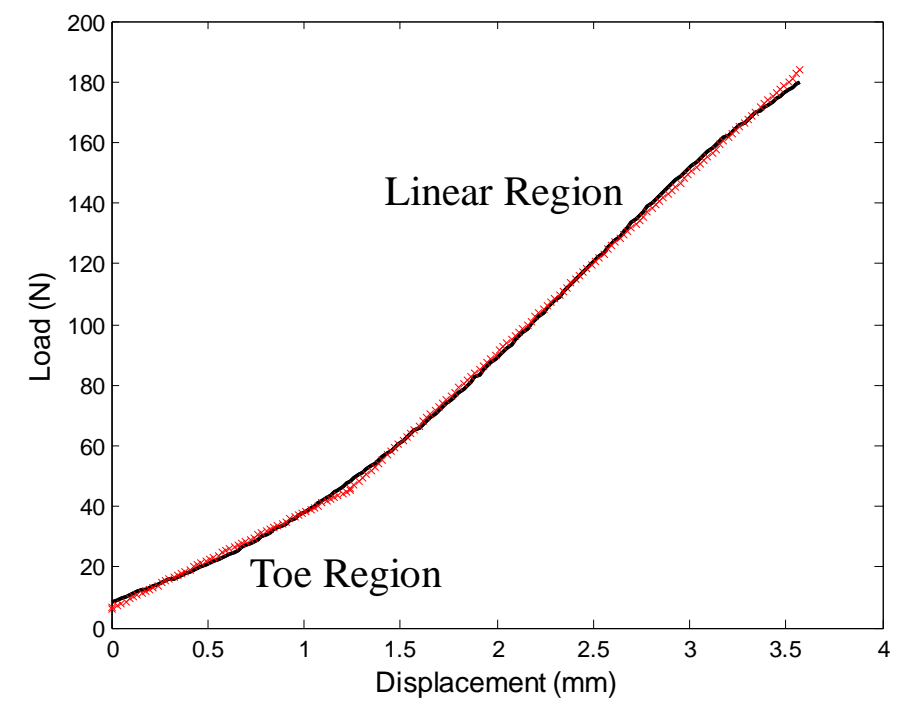

Figure 2.19- Curve after linear regression analysis. Red line denotes best fit line for the two regions.

The Intact specimens underwent a different linear regression analysis. Based on previous work, an exponential expression ${ }^{26}$ was used to fit the Intact curves (Equation 1). The 
coefficients are as defined: A being a material coefficient directly related to stress, B being the rate of change of the tangent modulus with respect to the stress and thought of as the rate of fiber recruitment for stretching of collagenous fibrous tissues, and $\mathrm{C}$ (product of $\mathrm{A}$ and $\mathrm{B}$ ) being the tangent modulus of the tissue as the stress approaches zero ${ }^{26}$. Using Matlab, the strain (x-axis) and the stress (y-axis) was the input and the equation was fit (by cftool code) over the original curve (Figure 2.20). The program outputted values for $A, B$, and a $\mathrm{R}^{2}$ to validate the accuracy of the fit. Then, the stress-strain curve was inputted into the TestWorks (MTS) to obtain a value for the Young's Modulus (E).

Equation 1: $\sigma=A\left(e^{B s}-1\right)$

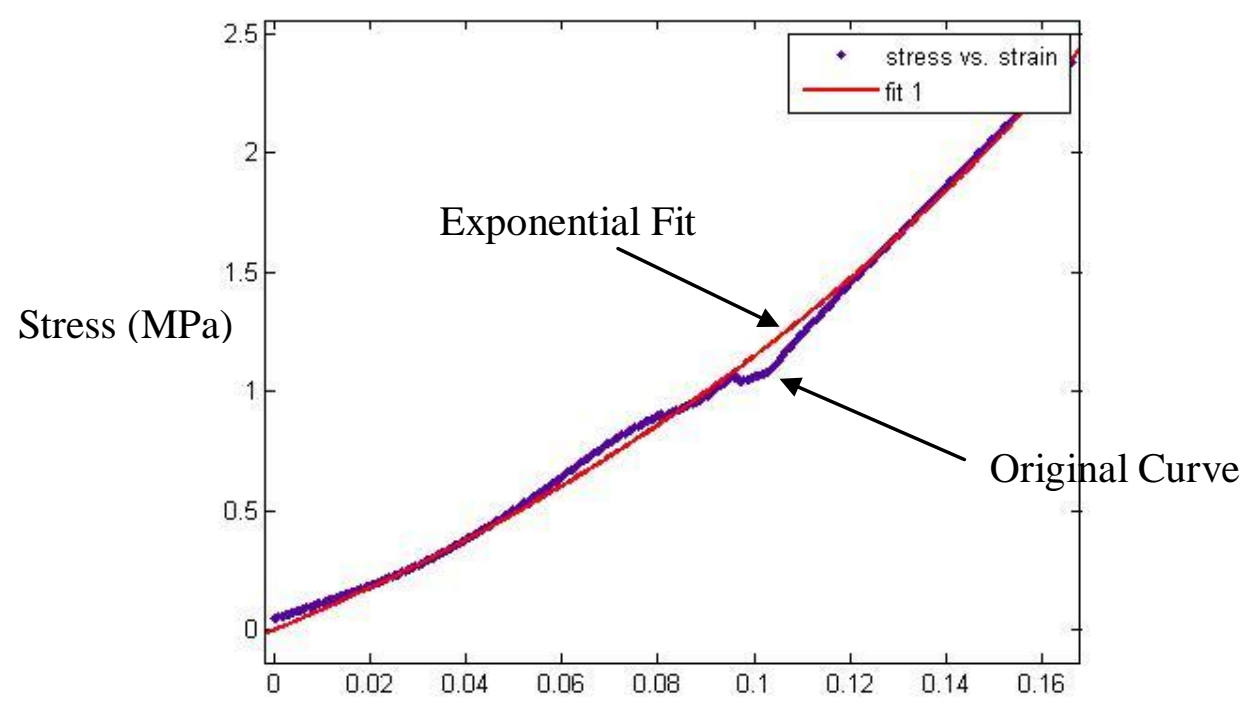

Strain

Figure 2.20- Exponential Curve Fitting. The original data (blue line) is fitted with the equation above (red line). 
Anatomical data was analyzed using a one-way analysis of variance (ANOVA) with repeated measures. The Tukey post-hoc test for multiple comparisons was used when significant differences among the experimental conditions were detected. A two-tailed, paired t-test was employed to compare the two repair constructs. The threshold for statistical significance was $\mathrm{p}<$ 0.05. Also, an ANOVA with repeated measures was used to compare the optical segment data.

\section{Section 3: Results}

\section{Anatomical Data}

Randomization resulted in no statistical difference in BMD between the DR-MCS (592.2 $\pm 258.3 \mathrm{HU})$, the DR-KLA $(558.4 \pm 254.4 \mathrm{HU})$, or the Intact $(627.9 \pm 165.1 \mathrm{HU})$ groups (Table 3.1). For tendon measurements, there was no significant difference in tendon width between the DR-MCS $(32.1 \pm 6.4 \mathrm{~mm})$, the DR-KLA $(30.9 \pm 4.3 \mathrm{~mm})$, or the Intact $(34.9 \pm 4.3 \mathrm{~mm})$. Also, there was no significant difference in tendon thickness between the DR-MCS $(6.7 \pm 1.5 \mathrm{~mm})$, the DR-KLA $(6.6 \pm 2.2 \mathrm{~mm})$, or the Intact $(5.5 \pm 0.7 \mathrm{~mm})$. There was no difference in cross sectional area between the DR-MCS $\left(218.6 \pm 83.3 \mathrm{~mm}^{2}\right)$, the DR-KLA $\left(198.6 \pm 65.8 \mathrm{~mm}^{2}\right)$, or the Intacts $\left(191 \pm 37.1 \mathrm{~mm}^{2}\right)$ Furthermore, there was no statistical difference in overall footprint length between the DR-MCS $(36.1 \pm 3.9 \mathrm{~mm})$ and the DR-KLA $(36.1 \pm 3.7 \mathrm{~mm})$. Considering all 12 repaired specimens, the superoposterior footprint length $(14.4 \pm 2.9 \mathrm{~mm})$ was significantly less than $(\mathrm{p}<0.05)$ the lateral $(18.3 \pm 3.6 \mathrm{~mm})$. 
Table 3.1- Anatomical Data for Repair Groups and Intact Specimens (Mean and standard deviation)

\begin{tabular}{|c|c|c|c|c|c|}
\hline Group & BMD (HU) & $\begin{array}{c}\text { Tendon } \\
\text { Width }(\mathrm{mm})\end{array}$ & $\begin{array}{c}\text { Tendon Thickness } \\
(\mathrm{mm})\end{array}$ & $\begin{array}{c}\text { Cross Sectional } \\
\text { Area }\left(\mathrm{mm}^{2}\right) \\
\end{array}$ & $\begin{array}{c}\text { Footprint } \\
\text { Length }(\mathrm{mm})\end{array}$ \\
\hline DR-MCS & $\begin{array}{c}592.2 \\
(258.3)\end{array}$ & $\begin{array}{l}32.1 \\
(6.4)\end{array}$ & $\begin{array}{c}6.7 \\
(1.5)\end{array}$ & $\begin{array}{c}218.6 \\
(83.3)\end{array}$ & $\begin{array}{l}36.1 \\
(3.9)\end{array}$ \\
\hline DR-KLA & $\begin{array}{c}583.6 \\
(254.4)\end{array}$ & $\begin{array}{l}30.9 \\
(4.3)\end{array}$ & $\begin{array}{c}6.6 \\
(2.2)\end{array}$ & $\begin{array}{l}198.6 \\
(65.8)\end{array}$ & $\begin{array}{l}36.1 \\
(3.7)\end{array}$ \\
\hline Intact & $\begin{array}{c}627.9 \\
(165.1)\end{array}$ & $\begin{array}{l}34.9 \\
(4.3)\end{array}$ & $\begin{array}{c}5.5 \\
(0.7)\end{array}$ & $\begin{array}{c}191 \\
(37.1)\end{array}$ & - \\
\hline
\end{tabular}

Mechanical Data: Intact Group

Table 3.2- Mechanical Data for Intact Specimens $(n=4)$

\begin{tabular}{cccccc} 
Specimen \# & $\begin{array}{c}\text { Maximum } \\
\text { Load } \\
(\mathbf{N})\end{array}$ & $\begin{array}{c}\text { Stiffness } \\
(\mathbf{N} / \mathbf{m m})\end{array}$ & $\begin{array}{c}\text { Displacement to } \\
\text { Max Load } \\
(\mathbf{m m})\end{array}$ & $\begin{array}{c}\text { BMD } \\
(\mathbf{H U})\end{array}$ & Failure Mode \\
\hline I-1 & 433 & 82.8 & 7.7 & 445.5 & Bone Fracture \\
$\mathbf{I - 2}$ & 1005 & 92.8 & 12.5 & 531.3 & Muscle Tear \\
I-3 & 2078 & 145.5 & 18.4 & 780.6 & Avulsion \\
I-4 & 1658 & 166.6 & 13.5 & 754 & Avulsion \\
Avg (SD) & 1294 & 121.9 & 13 & 627.9 & \\
\hline
\end{tabular}




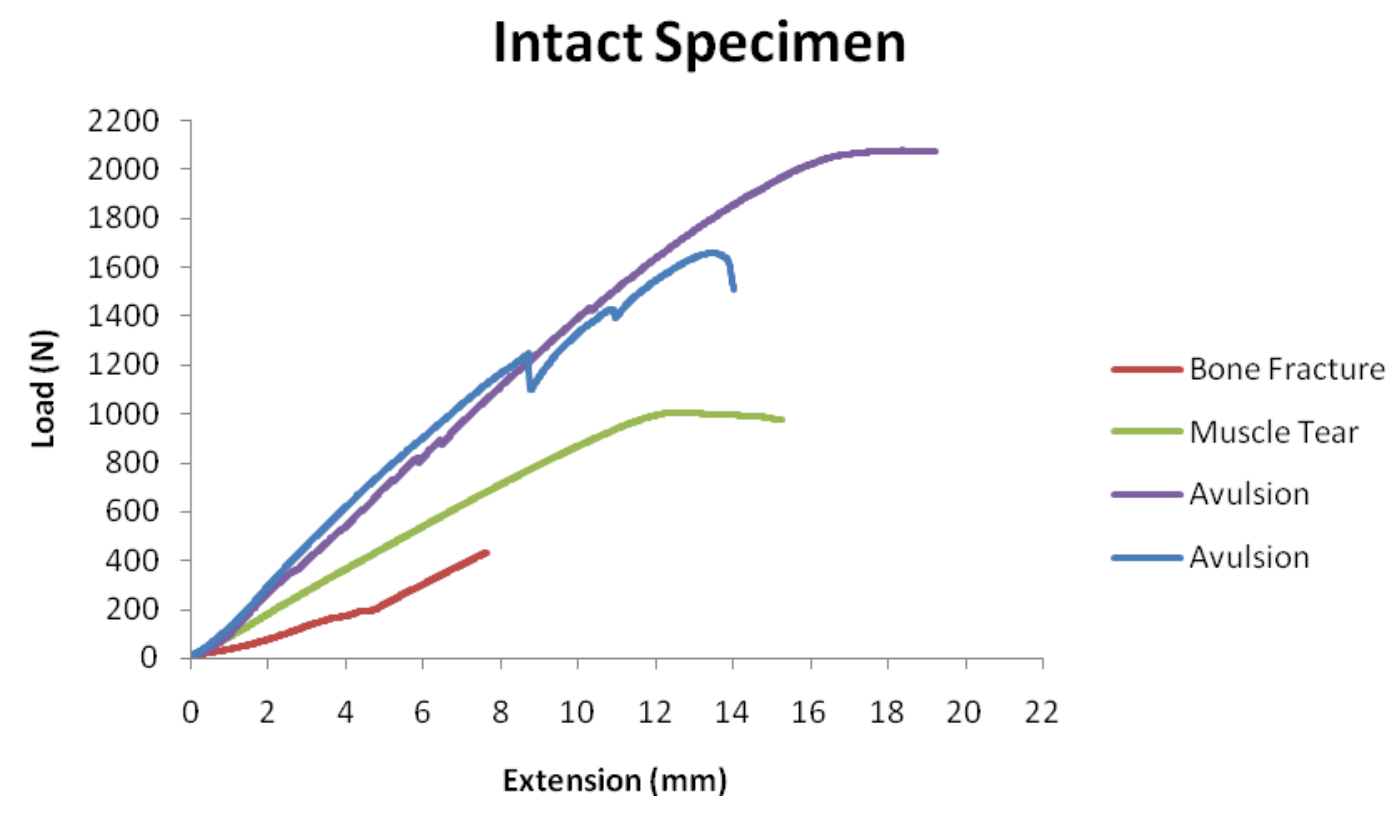

Figure 3.1- Intact load-extension curve for all specimens to maximum load. The specimen failing by bone fracture occurred at a low load and the one failing by avulsion occurred at a higher load.

In the Intact group, two specimens failed by avulsion, one specimen failed by tendon tear, and one specimen failed by trochanter bone fracture (entire greater trochanter fractured off). Avulsion is defined, in this case, as a tearing of tendon and bone from the main mass of bone. Bone fracture is defined as a large amount of bone cracking or breaking. The tendon tear occurred on the superoposterior side, starting above the markers and then propagated to the lateral side towards the insertion site. Also, the bone fracture occurred at lower loads, where the specimen BMD was on the lower end. After testing, the tendon and tendon-mucle unit were maintained at the acceptable temperature. Because of the different types of failure, the mechanical data (Table 3.2) and curves (Figure 3.1) for each specimen was slightly different. Though, the specimens that failed by avulsion demonstrated similar properties.

From the equation in the Methods, an exponential curve was fitted to the Intact 
specimens (Figure 3.2). The variables (Table 3.3) are A- a material coefficient, B- the rate of change of the tangent modulus with respect to the stress, and C- tangent modulus of the tissue as the stress approaches zero. To verify the fit is accurate, the $\mathrm{R}^{2}$ value was reported. Furthermore, based on the stress-strain curve the Young's modulus was defined, E, for each specimen.

Table 3.3- Curve fit data (coefficients A,B,and C) and Young's Modulus

\begin{tabular}{cccccc}
\hline Specimen & $\mathbf{A}$ & $\mathbf{B}$ & $\mathbf{C}$ & $\mathbf{R}^{\mathbf{2}}$ & $\mathbf{E}(\mathbf{M P a})$ \\
\hline $\mathbf{I}-\mathbf{1}$ & 1.36 & 6.10 & 8.30 & 0.997 & 26.4 \\
$\mathbf{I}-\mathbf{2}$ & 1.64 & 7.44 & 12.20 & 0.996 & 34.5 \\
$\mathbf{I - 3}$ & 2.27 & 4.20 & 9.54 & 0.983 & 39.4 \\
$\mathbf{I}-4$ & 2.63 & 5.95 & 15.65 & 0.978 & 37.4 \\
Average & 1.98 & 5.92 & 11.42 & 0.989 & 34.4 \\
& $(0.58)$ & $(1.33)$ & $(3.25)$ & $(0.01)$ & $(5.7)$ \\
\hline
\end{tabular}

Table 3.4- Exponential Coefficients for other tissues measured.

\begin{tabular}{cccccc}
\hline Tissue & Type & A & B & C & E (MPa) \\
\hline Glenohumeral $^{2}$ & \multirow{2}{*}{ Human } & 0.43 & 14.14 & 5.50 & 38.74 \\
ACL $^{27}$ & & \pm 0.24 & \pm 4.17 & \pm 2.73 & \pm 18.09 \\
& Rabbit & - & 81 & 68 & 674 \\
Patellar Tendon $^{27}$ & \multirow{2}{*}{ Rabbit } & - & \pm 12 & \pm 16 & \pm 62 \\
Tail Tendon $^{28}$ & \multirow{2}{*}{ Mouse } & 128 & 2.18 & 248 & \pm 132 \\
& & \pm 82 & \pm 0.84 & \pm 181 & - \\
\hline
\end{tabular}



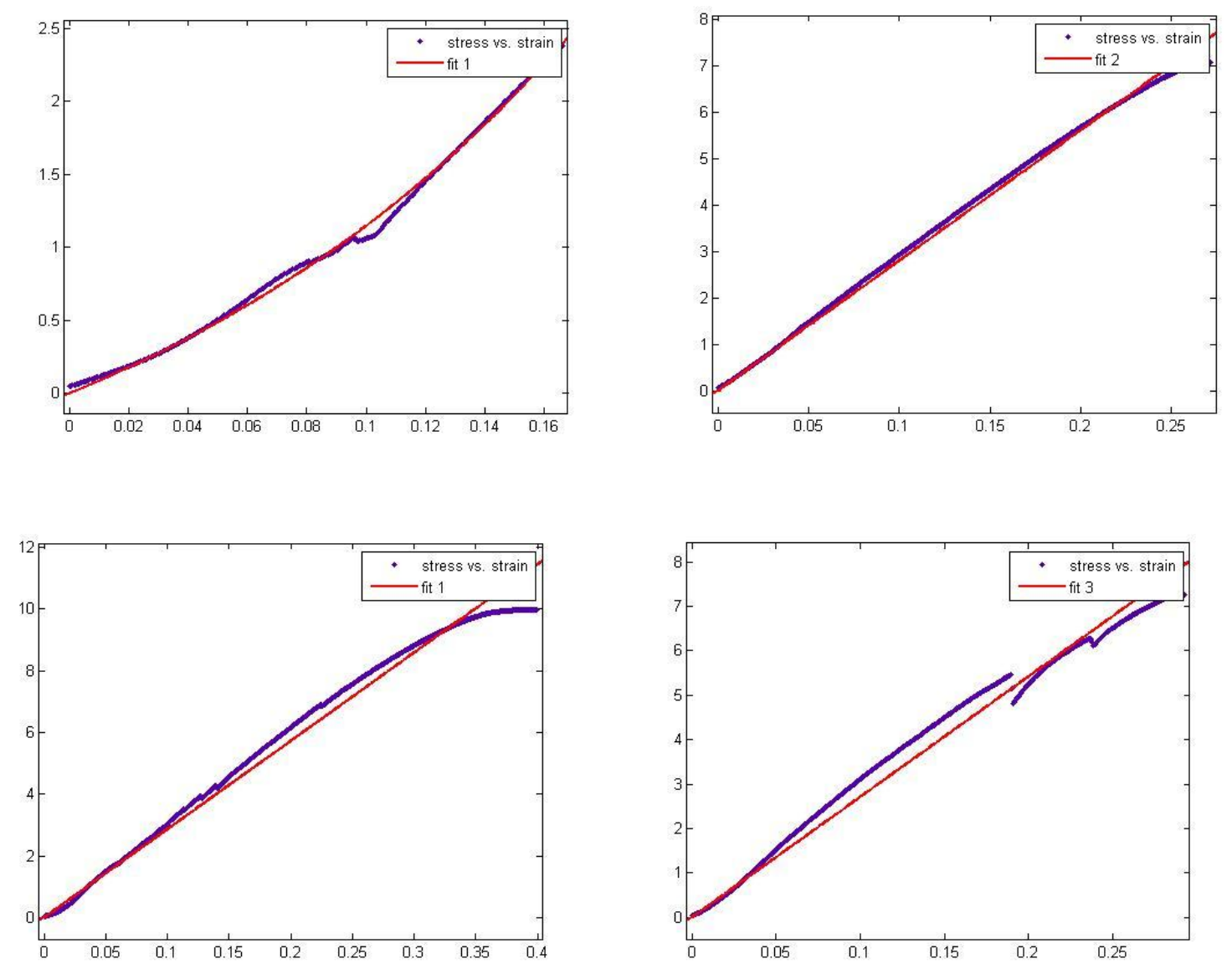

Figure 3.2- Exponential curve fitting. Top Row: I-1 and I-2. Bottom Row: I3 and I-4. Blue line represents the original stress-strain data and the red line represents the curve fit. Fit was very accurate.

The Intact optical data was analyzed by averaging the rows of markers across the tissue. As in the Methods, the tissue was divided into separate rows, which included Lower Trochanter (closest to the potted femur), Below Insertion (insertion site), Above Insertion (insertion site), and Tendon-Muscle (tendon-muscle junction). The values consist of (Figure 3.3): Lower Trochanter (3.54 $\pm 0.43 \mathrm{~mm})$, Below Insertion (5.24 $\pm 1.1 \mathrm{~mm})$, Above Insertion $(7.59 \pm 3.65 \mathrm{~mm})$, and Tendon Muscle $(10.68 \pm 3.88 \mathrm{~mm})$.

Additionally, averaged relative segment elongation (RSE) at maximum load was found. 
This index was computed for segments of the tendon-bone and at the insertion site on the superoposterior (SP) and lateral side. Only three specimens were used because one specimen failed at a much lower load $(433 \mathrm{~N})$. The results are (Figure 3.4): Lateral tendon-bone (3.51 \pm 0.64\%), SP tendon-bone ( $7.15 \pm 0.34 \%)$, Lateral insertion (1.69 $\pm 0.93 \%)$, and SP insertion (3.59 $\pm 0.32 \%)$.

\section{At Max Load}

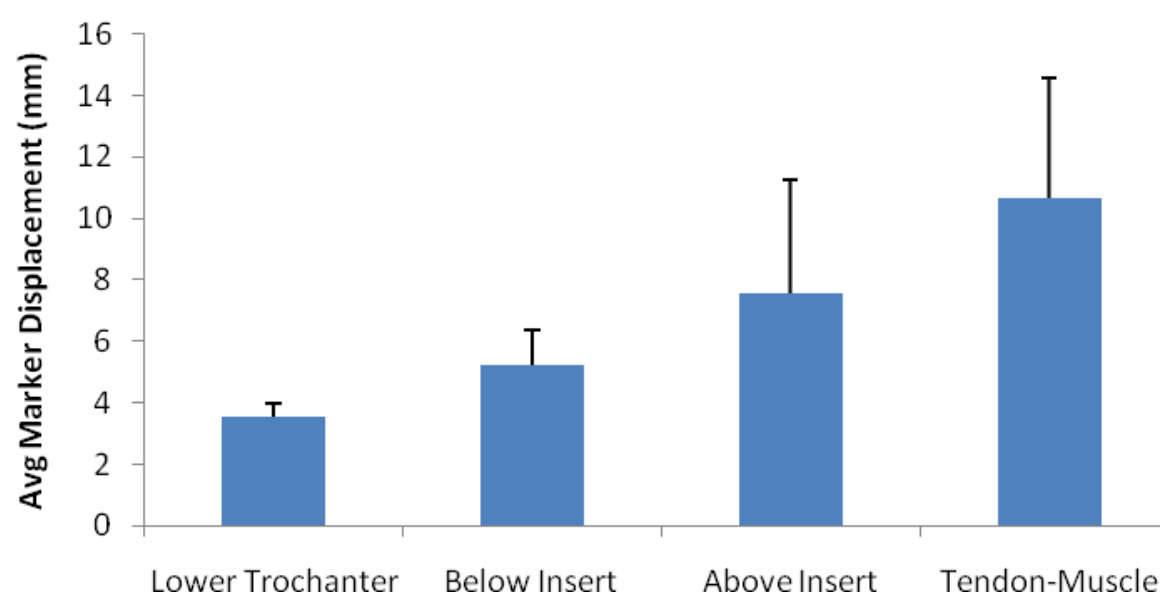

Figure 3.3- Averaged marker displacement based on rows. The superior markers elongate the most (tendon-muscle). 


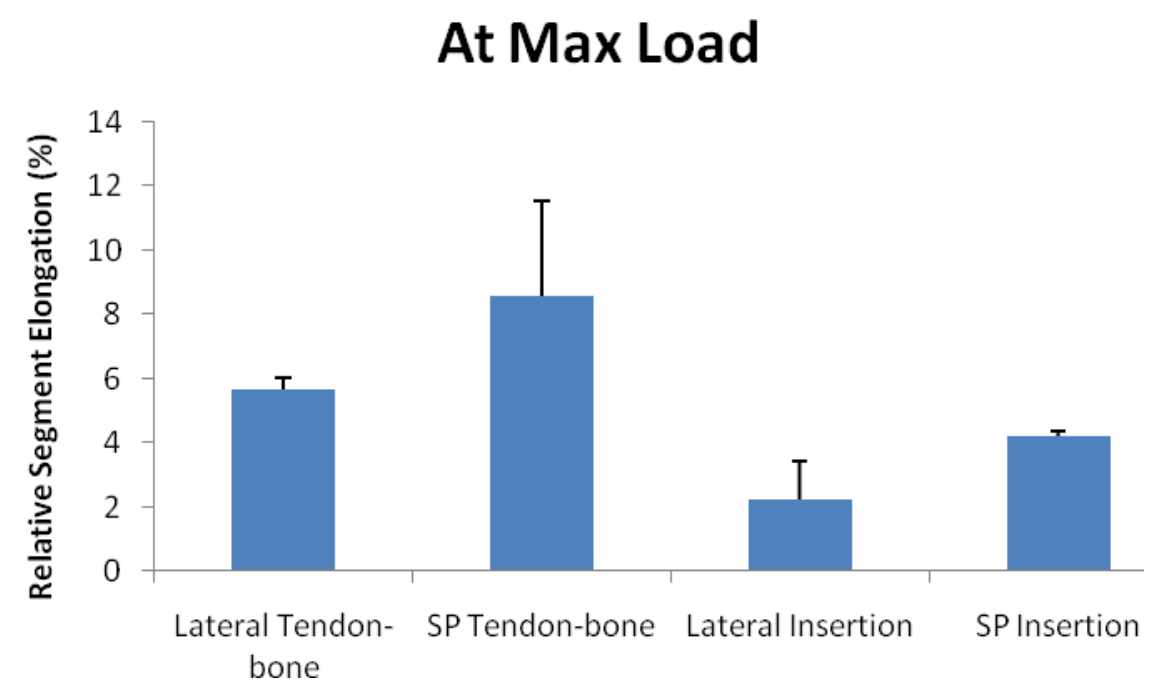

Figure 3.4- RSE of the different sides at max load. There is a greater elongation on the SP side during loading.

Based on the Intact mechanical data, the maximum load was used to design a cyclic test protocol for the repair groups. The peak cyclic load should exist beyond the toe region to ensure that the tendon fibers are uncrimped and undergo some tension. Also, this load should exist in the lower linear region as to load the tendon fibers (begin to become parallel) and not to damage any tissue (plastic region).

\section{Mechanical Data: Repair Groups}

The cyclic test data was obtained from the MTS and analysis was perfomed based on the $1^{\text {st }}$ and last cycle. The last cycle correlates to the viscoelastic properties of the tendon. The tendon and repair, at this point, have reached a cyclic creep (mechanical) steady state. There was no significant difference found in secant stiffness for the $1^{\text {st }}$ cycle $(\mathrm{p}=0.99)$ or the last cycle $(\mathrm{p}=0.83)$, cyclic elongation $(\mathrm{p}=0.84)$, or elongation amplitude $(\mathrm{p}=0.89)$ between the repair groups (Table 3.4). There is a significant difference between the secant stiffness of the $1^{\text {st }}$ and the last 
cycle for the DR-MCS and DR-KLA.

Table 3.5- Cyclic data from MTS. $\alpha$ denoted significant difference among a group.

\begin{tabular}{ccccc}
\hline Group & $\begin{array}{c}\text { Sect Stiff } \\
\text { 1st cycle } \\
(\mathbf{N} / \mathbf{m m})\end{array}$ & $\begin{array}{c}\text { Sect Stiff } \\
\text { Last cycle } \\
\mathbf{( N / m m )}\end{array}$ & $\begin{array}{c}\text { Cyclic } \\
\text { Elongation } \\
(\mathbf{m m})\end{array}$ & $\begin{array}{c}\text { Elongation } \\
\text { Amplitude } \\
(\mathbf{m m})\end{array}$ \\
\hline \multirow{2}{*}{ DR-MCs } & 47.18 & $26.56^{\alpha}$ & 2.98 & 2.72 \\
& $(2.68)$ & $(9.28)$ & $(1.78)$ & $(0.17)$ \\
DR-KLA & 48.55 & $26.52^{\alpha}$ & 2.80 & 2.69 \\
& $(7.33)$ & $(5.46)$ & $(0.81)$ & $(0.41)$ \\
\hline
\end{tabular}

Linear regression analysis was performed for the cyclic loading test and assessed from initiation to the peak of the cycle. The data consisted of load and extension (MTS). There was no significant difference in toe region length between the DR-MCS $(1.03 \pm 0.07 \mathrm{~mm})$ and the DRKLA $(0.96 \pm 0.12 \mathrm{~mm})$. Though, the DR-KLA $(22.25 \pm 2.99)$ has a significantly higher toe region slope than the DR-MCS $(18.18 \pm 0.94)$. The DR-KLA, as it is loading, may be more resistant to the applied load. There was no significant difference in linear region slope between the DR-MCS $(38.30 \pm 2.8)$ and the DR-KLA (39.70 \pm 5.09$)$.

Following the cyclic loading test, load to failure data was obtained and compared between the two repairs. There was no difference in maximum $(\mathrm{p}=0.88)$ or yield load $(\mathrm{p}=0.18)$ between repair techniques. There was a significant difference $(\mathrm{p}<0.05)$ in normalized yield load (normalized with respect to max load), where this parameter was higher for DR-KLA (63.8 \pm $21.6 \%)$ relative to DR-MCS $(45.7 \pm 7.0 \%)$. Similarly, normalized work to yield load for the DRKLA $(40 \pm 16.1 \%)$ was significantly higher than the DR-MCS $(11.9 \pm 5.5 \%)$. Linear stiffness for the DR-MCS $(54.9 \pm 3.8 \mathrm{~N} / \mathrm{mm})$ was significantly higher than that of the DR-KLA (43.4 \pm 14.1N/mm) (Table 3.5). Post yield extension (crosshead extension from yield to max load) of the DR-KLA (7.2 $\pm 3.4 \mathrm{~mm})$ was significantly less than that of the DR-MCS $(12.2 \pm 2.4 \mathrm{~mm})$. 
Furthermore, the curves of the DR-MCS and DR-KLA (Figure 3.5) will demonstrate the differences in failure mode, stiffness, and yield point.

Table 3.6- Mechanical Data of the Repair Groups. Asterisk denotes significant difference between groups $(\mathrm{p}<0.05)$.

\begin{tabular}{ccccccc}
\hline Repair & $\begin{array}{c}\text { Max Load } \\
(\mathbf{N})\end{array}$ & $\begin{array}{c}\text { Yield } \\
\text { Load (N) }\end{array}$ & $\begin{array}{c}\text { Stiffness } \\
(\mathbf{N} / \mathbf{m m}) *\end{array}$ & $\begin{array}{c}\text { Post Yield } \\
\mathbf{E x t} \\
(\mathbf{m m}) *\end{array}$ & $\begin{array}{c}\text { Norm } \\
\text { Yield Load } \\
(\%) *\end{array}$ & $\begin{array}{c}\text { Norm } \\
\text { Work to } \\
\text { Yield } \\
(\%) *\end{array}$ \\
\hline DR-MCS & 439 & 192 & 54.9 & 12.2 & 45.7 & 11.9 \\
& $(127)$ & $(18)$ & $(3.8)$ & $(2.4)$ & $(7.0)$ & $(5.5)$ \\
DR-KLA & 454 & 269 & 43.4 & 7.2 & 63.8 & 40 \\
& $(179)$ & $(107)$ & $(14.1)$ & $(3.4)$ & $(21.6)$ & $(16.1)$ \\
\hline
\end{tabular}

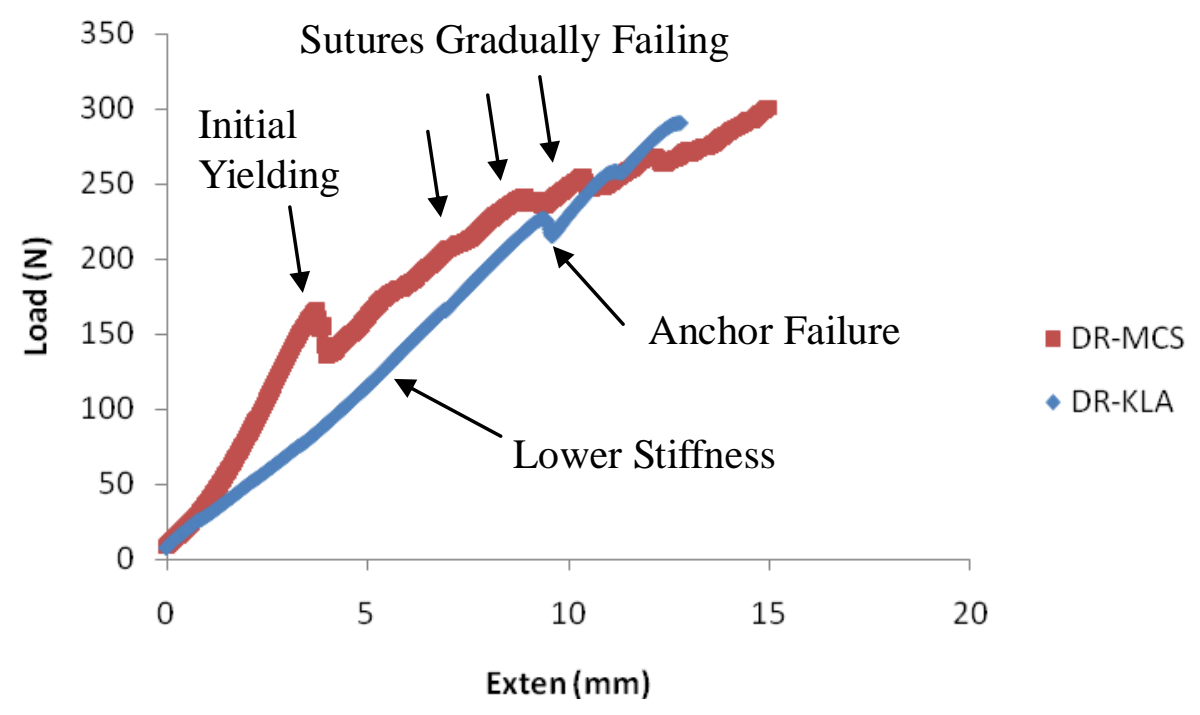

Figure 3.5- Representative loading curves for repair groups. DR-MCS demonstrates initial yielding and a greater stiffness.

In the DR-MCS repair group, four of the six specimens failed on the superoposterior side 
by the suture pulling through the tendon and two failed on the lateral side by similar means

(Figure 3.6). For the DR-KLA repair group, there were three specimens that failed on the superoposterior side by anchor pullout of the bone, one by suture pullout of the anchor and one on the lateral side by anchor pullout (Figure 3.7). One specimen failed during the cyclic test by anchor pullout due to redrilling of anchor holes, and therefore was omitted from the results. The anchors were misaligned in the bone, taken out, and the hole was made larger. Thus, the anchor came loose because it was not tightly placed in the bone.

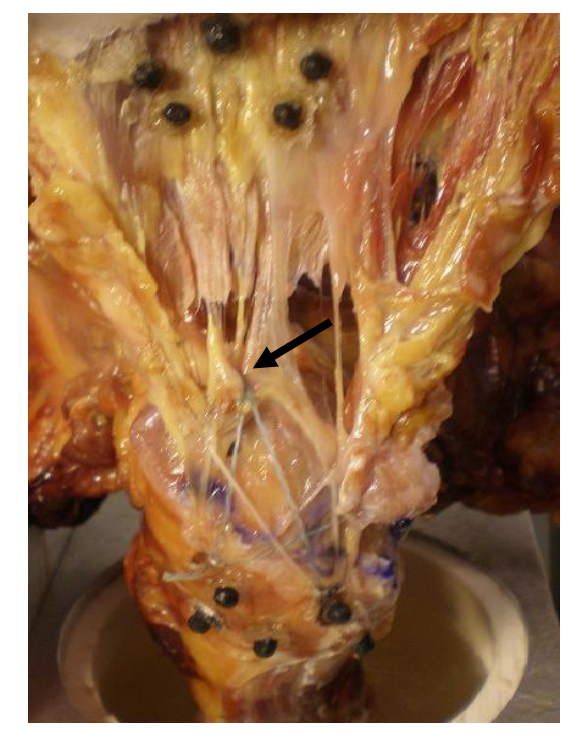

Figure 3.6- DR-MCS failure occurred by suture pulling through the tendon.

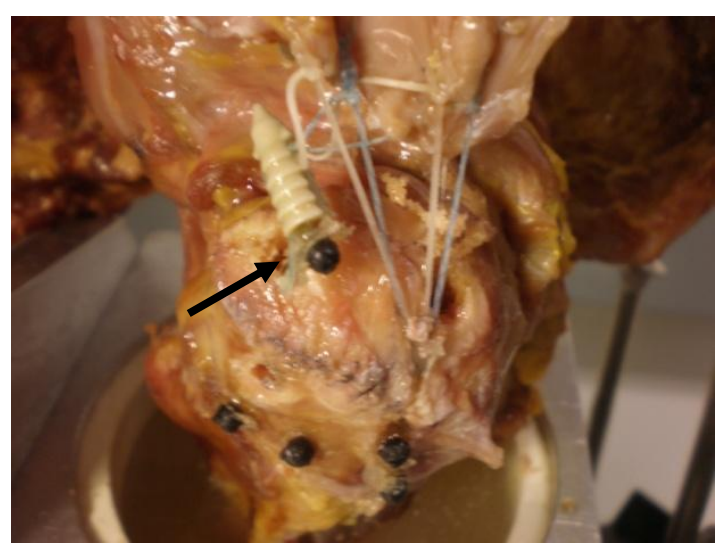

Figure 3.7- DR-KLA failure occurred by anchor pullout.

Similar to the cyclic loading test, a linear regression analysis was performed on the pull to failure test data. For each specimen, the load and extension data of the MTS from initiation to yield load was used. The DR-KLA $(2.53 \pm 1.19 \mathrm{~mm})$ showed a significantly greater toe region length than the DR-MCS $(1.38 \pm 0.17 \mathrm{~mm})$ (Figures 3.8). There was no significant difference in toe region slope between the DR-MCS $(31.08 \pm 2.89 \mathrm{~N} / \mathrm{mm})$ and the DR-KLA $(26.82 \pm 4.75$ $\mathrm{N} / \mathrm{mm}$ ). There was a significant difference in linear region slope between the DR-MCS (54.27 \pm $4.01 \mathrm{~N} / \mathrm{mm})$ and the DR-KLA $(42.91 \pm 14 \mathrm{~N} / \mathrm{mm})$. To verify that the linear region slope was 
acceptable, the MTS stiffness was statistically compared. There was no significant difference in the DR-MCS $(\mathrm{p}=0.79)$ or the DR-KLA $(\mathrm{p}=0.95)$.

(a)

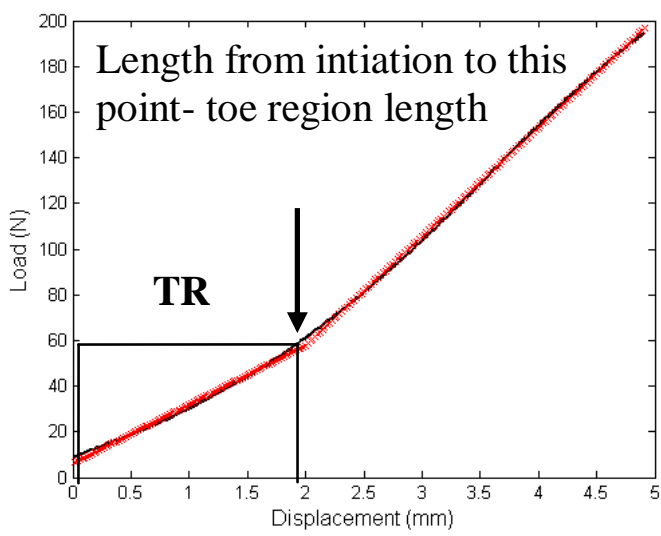

(b)

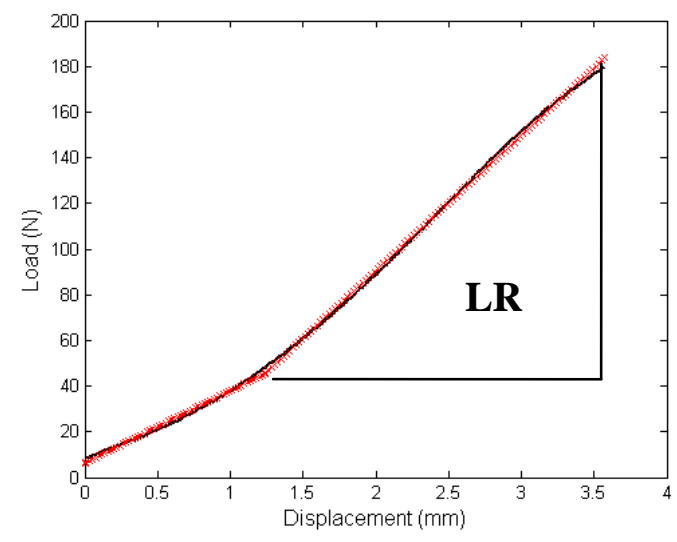

Figure 3.8- Slopes of the (a) DR-KLA and (b) DR-MCS repairs. The toe region length is smaller in the DR-MCS, while the linear region slope is greater than the DR-KLA $\mathrm{TR}=$ toe region, $\mathrm{LR}=$ linear region slope.

\section{Optical Data: Repair Group}

Optical data was based on the relative segment elongation (RSE) for segments of the repair-tendon unit (RTU) and the muscle-bone (MB). Cyclic elongation was calculated for the last cycle for each group of segments The averaged RSE for the RTU (Figure 3.9) of the DRMCS $(26.6 \pm 8.9 \%)$ was significantly higher than the RTU of the DR-KLA $(19.0 \pm 5.3 \%)$. No significant differences in RSE for the MB between the repair groups were found. This may be due to the organization of the tendon-repair, in which it takes further loading to reach a tensile state. 


\section{Cyclic Elongation of RTU}

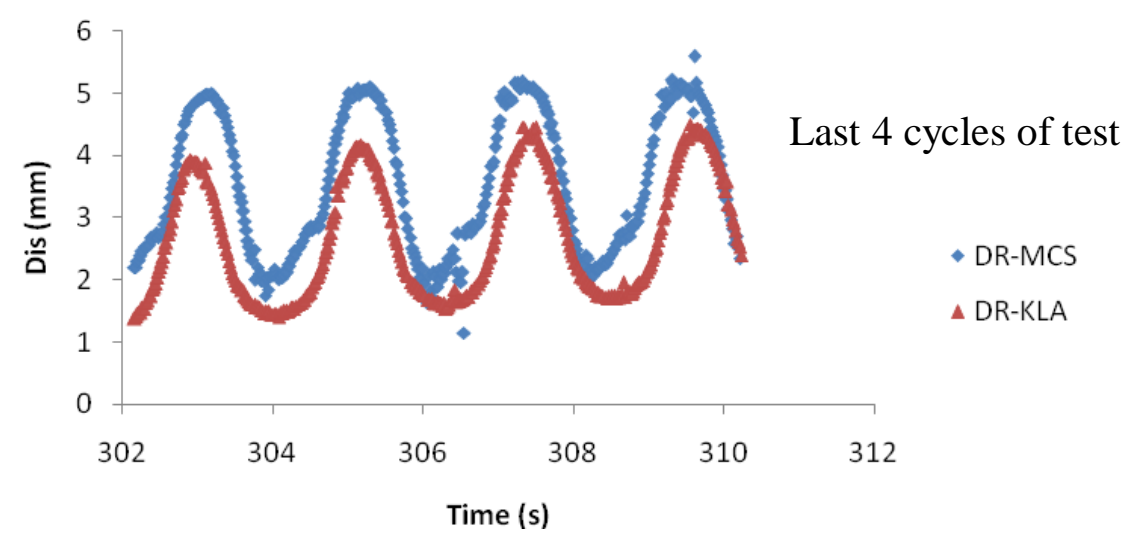

Figure 3.9- An optical data sample of the RSE for the RTU. From the averaged RTU, the DR-MCS has a greater cyclic elongation.

At yield load, the averaged RSE for the RTU $(13.9 \pm 3.3 \%)$ and the MB $(11.6 \pm 3.7 \%)$ of the DR-KLA was significantly higher than the RTU $(9.9 \pm 3.5 \%)$ and MB $(7.1 \pm 2.7 \%)$ of the DR-MCS (Figure 3.10). At max load, there was no significant difference in RSE between repair groups for the RTU or the MB. The post yield extension (Figure 3.11) for the RTU (12.7 $\pm 9.6 \%$ ) and the MB $(23.9 \pm 13.8 \%)$ of the DR-KLA was significantly less than the RTU $(22.5 \pm 8 \%)$ and $\operatorname{MB}(31.7 \pm 11.2 \%)$ of the DR-MCS. 

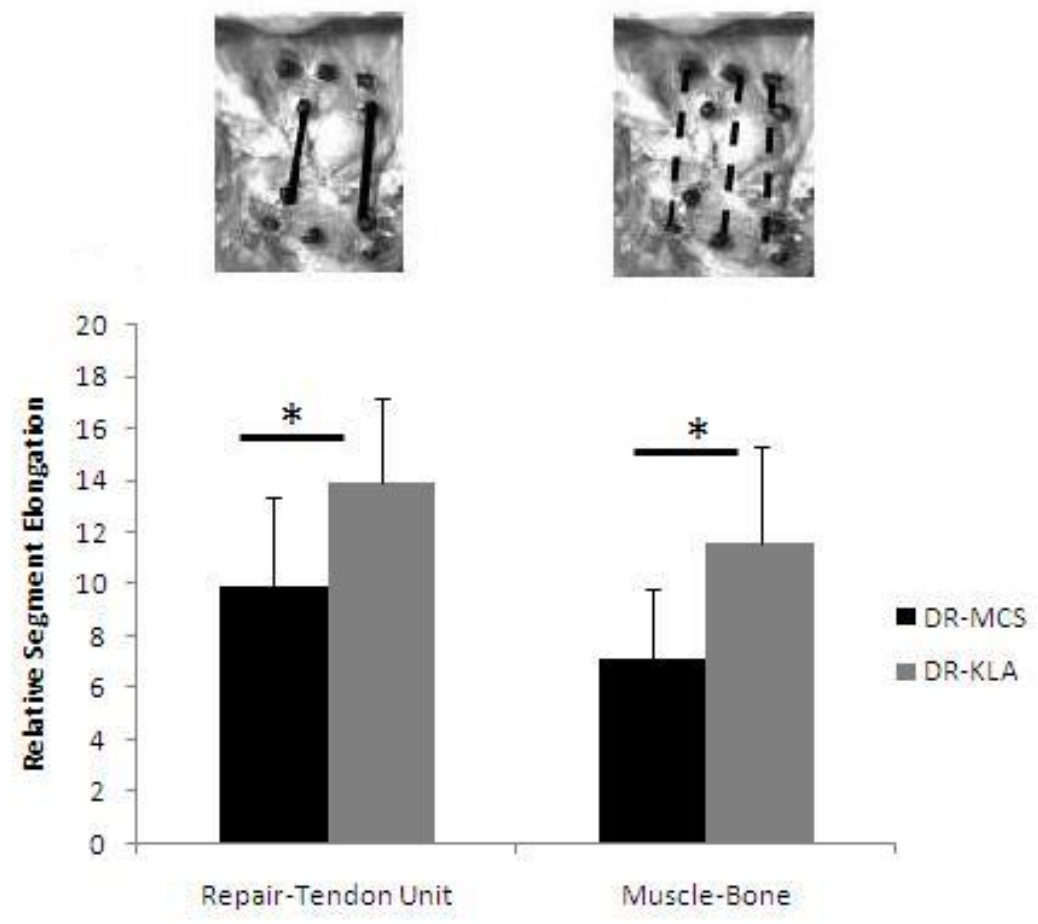

Figure 3.10- Averaged RSE at yield load. Left image depicts the RTU and righ image depicts the $\mathrm{MB}$.

\section{Post Yield Ext for RTU}

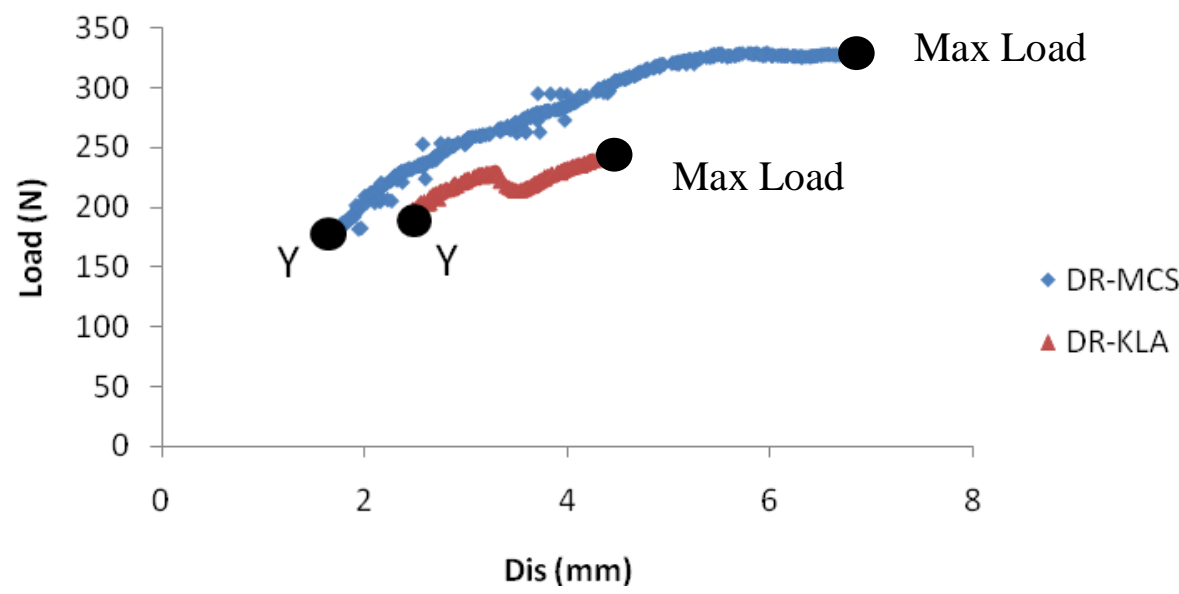

Figure 3.11- Sample optical data of the post yield extension for the RTU. After yield (Y), the DR-MCS extended a large amount compared to the DR-KLA to max load. 
Each segment was analyzed to distinguish any variation across the tissue within a repair. No significant difference was found between any of the segments for the DR-KLA or DR-MCS at yield. At max, there was a significant difference only in the RTU on the lateral side for both repairs. This means that the lateral side RTU elongated significantly further in the DR-KLA and the DR-MCS than all other segments.

\section{Correlation Factor}

The maximum load was strongly correlated to BMD for the DR-KLA $\left(\mathrm{R}^{2}=0.816\right)$, but not for the DR-MCS $\left(\mathrm{R}^{2}=0.004\right)$ (Figure 3.12). Furthermore, the maximum load has a strong correlation to tendon thickness for the DR-KLA $\left(\mathrm{R}^{2}=0.896\right)$, but not for the DR-MCS $\left(\mathrm{R}^{2}=0.424\right)$ (Figure 3.13). Tendon thickness was measured before repair and for the area just before the insertion into the muscle. There was no correlation between max load and tendon width for the DR-MCS or the DR-KLA

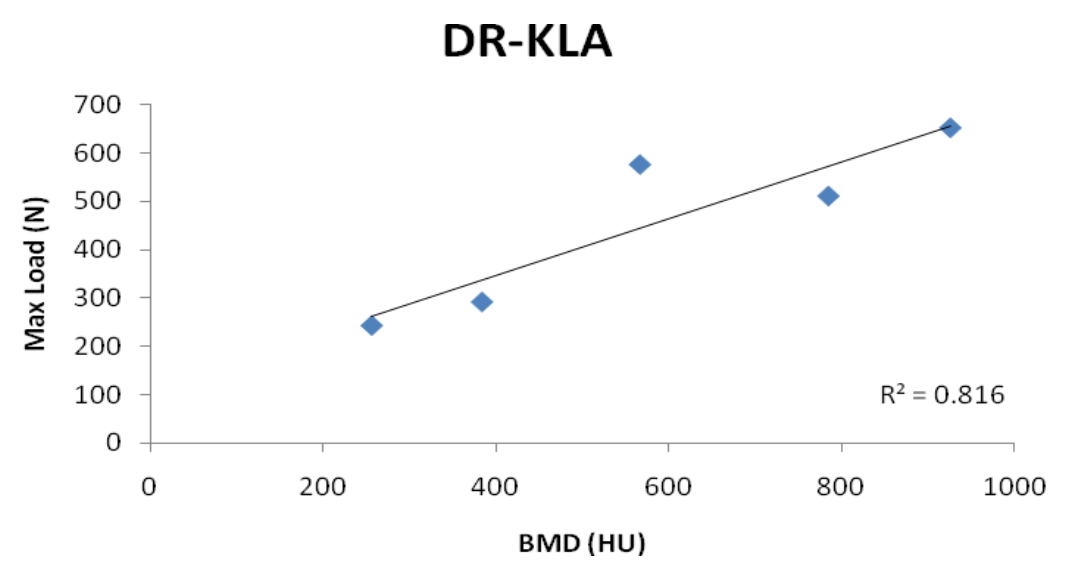




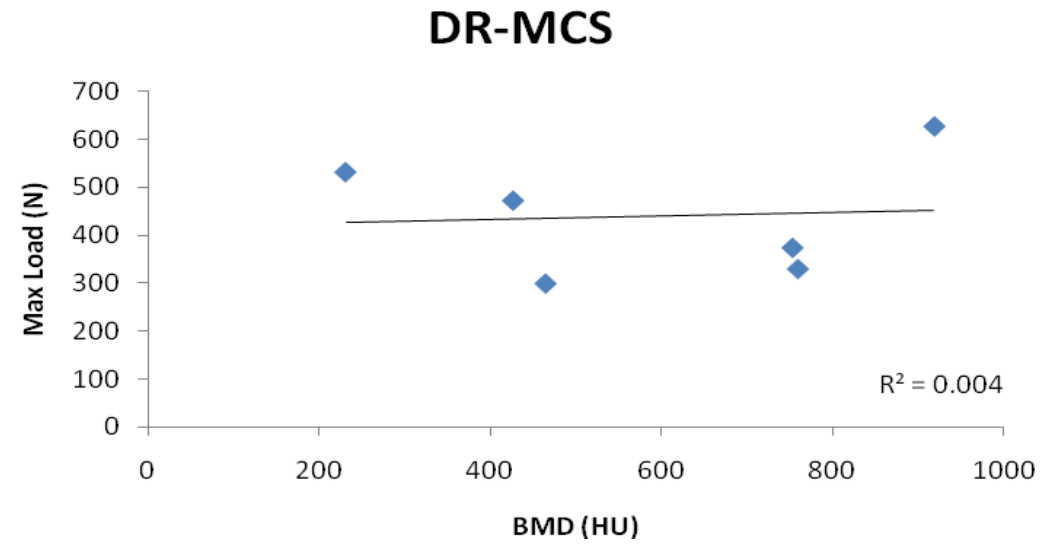

Figure 3.12- DR-KLA and DR-MCS correlation. Max load is correlated to BMD in the DR-KLA. Specimens with higher BMD have a higher max load.

\section{DR-KLA}

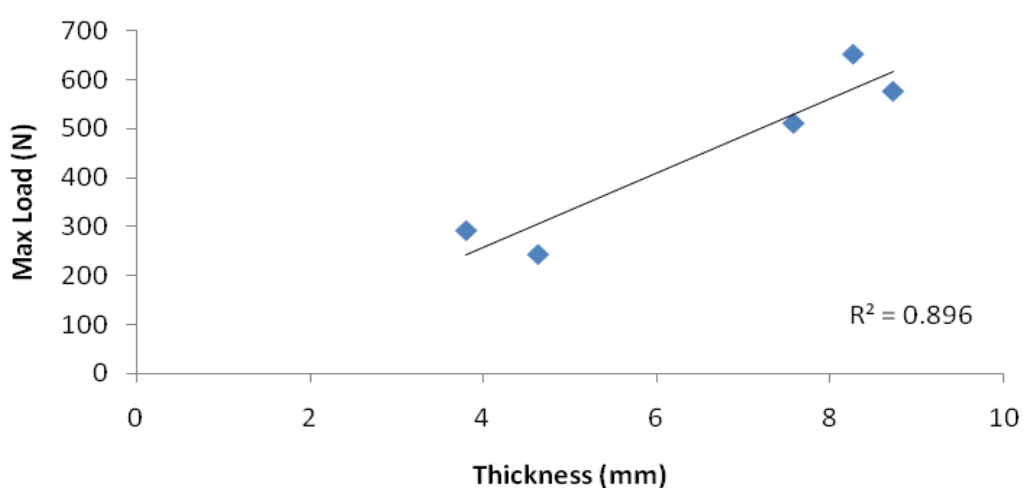




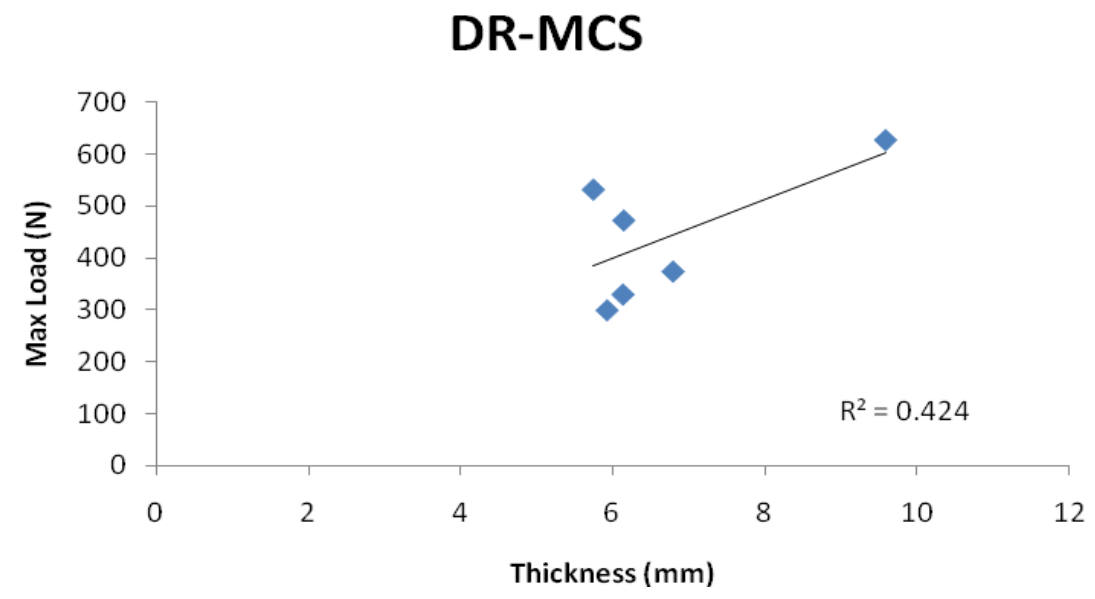

Figure 3.13- DR-KLA and DR-MCS correlation plot. Max load is correlated to thickness in the DR-KLA. Specimens with higher thickness have a higher max load.

\section{Section 3: Discussion}

Summary Table of significant differences found between repair groups.

\begin{tabular}{|l|l|l|}
\hline \multicolumn{1}{|c|}{ Anatomical } & \multicolumn{1}{c|}{ Cyclic Test for Repairs } & $\begin{array}{l}\text { DR-MCS: } \\
\text {-Higher stiffness } \\
\text {-Higher linear region slope } \\
\text {-Larger post yield extension } \\
\text {-Superoposterior footprint } \\
\text { length greater than lateral }\end{array}$ \\
$\begin{array}{l}\text {-Larger post yield segment } \\
\text { elongation }\end{array}$ \\
$\begin{array}{l}\text { - Higher relative segment } \\
\text { elongation of the RTU }\end{array}$ \\
$\begin{array}{l}\text { DR-KLA: } \\
\text {-Higher normalized yield load } \\
\text {-Higher relative segment } \\
\text { elongation of the RTU and MB }\end{array}$ \\
\hline
\end{tabular}


Repair of the gluteus medius (GM) is necessary at times, where pain is persistent and normal function is inhibited. There may be some difficulties in repairing tears of the tendons because of the differing footprints ${ }^{2}$, tear sites ${ }^{2,3,8,9}$, and lack of well established repair methods $s^{7,8,10,21}$. The shape, size, and amount of retraction of the tear may cause problems with surgical intervention at the site, repair reattachment, and repair time because the tendon's area may be compromised. Thus difficulties may occur in placing suture anchors around the footprint ${ }^{7,21}$ and regain full footprint area.

Although similarities have been drawn between the GM and rotator cuff ${ }^{3,8,9}$, biomechanical data of the GM is unknown. This association is based on the similar characteristics such as the tears increasing incidence with age, the portrayal of mature, rolled edges, causing weakness, radiological appearances, fatty infiltration in the muscle, and difficulty of repair. Surgical management of rotator cuff tendon tears are reasonably well established unlike the case for the GM. Hence, repair techniques for GM tears have followed similar approaches to those used for the rotator cuff. Clinically, these have been proven beneficial and increase range of motion while reducing pain. Due to the size and strength of the GM, clinicians have moved straight to Double Row techniques along with modified versions that use mattress and simple stitching with suture anchors or add knotless anchors to the stitch configuration, as opposed to Single Row techniques. So, rotator cuff repar techniques have evolved and have been redefined based on increasing strength of the repair and tendon pressure on the footprint. Before appropriate repairs are performed and evaluated, the intact GM muscles-tendon construct should be mechanically understood, as to investigate loaded native tissue.

The present study was undertaken to evaluate the properties of the intact GM muscletendon-bone unit and compare the biomechanical fixation characteristics of two specific GM 
repair techniques. Though the DR-KLA and DR-MCS are relatively strong (439 and 454 N, respectively), mechanical properties of these repair constructs are inferior to those of the intact unit (Table 3.2). The maximum loads of DR-KLA and DR-MCS repairs are $\sim 35 \%$ of the Intacts $(1293 \pm 723 \mathrm{~N})$. The stiffness of DR-KLA and DR-MCS repairs are $\sim 36 \%$ and $\sim 45 \%$, respectively of the Intacts $(121.9 \pm 40.5 \mathrm{~N} / \mathrm{mm})$. Direct comparison is not warranted, since cyclic loading preceded load to failure test of the repair group. For the specimen failing by bone fracture, the lower BMD was the underlying factor. Although this is a cadaveric model, a desirable repair would be as mechanically strong and stiff as the native tissue. To our knowledge, biomechanical properties of the native gluteus medius tendon have not been reported.

Based on the optical data of the Intact group, it is apparent that the elongation is greater (Figure 3.3) near the insertion site $(7.59 \mathrm{~mm})$ and on the tendon muscle junction $(10.68 \mathrm{~mm})$. These findings are in conjunction with how the tears of the gluteus medius muscle commonly occur by avulsion, in which the tear may occur at the insertional footprint or by separation of the muscle flaps at the tendon-muscle junction. Furthermore, during loading, the native tissue demonstrated variation between the lateral and superoposterior side. Under the prescribed loading condition, the superorposterior side has a greater elongation (across the tendon and the insertion site) than the lateral (Figure 3.4). So, during normal activity, the muscle may deform on the superoposterior side at first onset of loading. Based on the current setup, this could be a result of how the muscle was loaded. It can be presumed that the superoposterior side activates first then the lateral side accounts for abduction and allows external rotation during the swingthrough of walking ${ }^{2}$. The lateral side may undergo more loading anatomically, though; these actions are not accounted for. So, even though the superoposterior side elongates more, the lateral side may be subject to a large load. 
The repair groups demonstrated similarities in mechanical cyclic loading response, in which all parameters (Table 3.4) measured (cyclic elongation, elongation amplitude, and secant stiffness) showed no statistical difference between groups. So, both repairs indicated similar properties during cyclic loading, which can translate to strong stability properties during early rehabilitation. The optical findings, on the other hand, demonstrated a difference in cyclic elongation of the repair-tendon unit (RTU) between the repair groups. After cyclic loading, the cyclic elongation of the RTU for the Double Row with massive cuff stitch (DR-MCS) (26.6\%) was significantly greater than the Double Row with Knotless Lateral Anchors (DR-KLA) (19.0\%). These results may show that the repair-tendon unit is affected throughout cyclic loading due to the fact that no difference was found for similar optical analyses of muscle-bone (MB) segments. This will give better insight into the repair-tendon construct's local properties as if to investigate motion between the tendon and bone postoperatively and in vivo.

The DR-KLA would appear to be more resistant and allow motion within the repairs capability. Clinically, it would be desirable to have a construct that is well fixed and can be loaded with no concern of laxity or excess range of motion. Also, the DR-KLA provides better construct stabilization throughout cyclic loading. With the increased elongation in the DR-MCS, this can cause potential damage or rupture of the repair and inhibit proper healing. The better the retention properties, the less likely the construct will fail. Also, the tendon will not be subject to the entire load.

During the load to failure test, the DR-KLA repair exhibited its first signs of yield closer to the point of complete failure by anchor pullout (norm yield: 63.8\%), while the DR-MCS repair showed initial failure earlier $(45.7 \%)$ by suture rupture. Post yield extension (PYE) results were consistent with the normalized yield data, as the DR-MCS repair showed a mean PYE of 12.2 
mm compared to the DR-KLA of 7.2mm (Table 3.5). However, the DR-KLA repair-tissue construct may deform more when loaded due to its lower stiffness $(43.4 \mathrm{~N} / \mathrm{mm})$ compared to the DR-MCS (54.9 N/mm). Furthermore, the linear region slope was greater in the DR-MCS, which is consistent with the stiffness from the MTS (Figure3.8). Therefore, the DR-MCS is more resistant to loading and the fixation may have stronger suture retention properties.

The optical data adds further appreciation to these repairs. Namely, the DR-KLA exhibited a significantly higher RSE than the DR-MCS at yield for the RTU and MB (Figure 3.10). Also, the post yield segment elongation is similar to that of the mechanical PYE. When increased loading occurs, the DR-KLA will yield (initially fail) at a greater elongation. This point may relate clinically because, based on patient activity, the muscle will undergo small to more strenuous loading. Thus, a repair that can stretch under large loads without failure, may allow the patient to be more active during rehabilitation. For more strenuous rehabilitation, the repairtendon unit can slowly reach a greater range of motion. Furthermore, the strong fixation of the DR-KLA allowed for failure to occur at higher loads. When initial failure occurs in the DRMCS, the tendon will excessively stretch causing further damage as the tendon tears through the sutures gradually.

The significance of the differences between the DR-KLA and DR-MCS suggest that there was no difference in terms of maximum load to failure between the two repair groups after cyclical loading. In vivo studies are required to determine the differences in strain and healing attributes of each repair. The current study does demonstrate that the initial fixation properties of the two constructs are acceptable to be used. Both show that the entire construct has the potential to withstand early rehabilitation and progress to more rigorous exercises based on the cyclic and failure loading. 
Therefore, the data is clinically relevant with regard to the immediate postoperative period when considerably lower forces would be expected. In theory, the patient will have minimal loading applied to the tendon from the time immediately post-operatively until rehabilitation. Then, small loads ( 20lbs) will be applied to the lower extremity with the help of crutches ${ }^{21}$. Furthermore, active range of motion exercise will begin during a 2 month period. Following repair incorporation and successful insertion site healing, the structural properties of the reconstructed gluteus medius would be expected to increase.

After measuring the anatomical data and performing the testing sequence, some conclusions can be drawn between the two. The anatomical data demonstrates that the superoposterior facet is smaller than the lateral facet. This agrees with Robertson et $\mathrm{al}^{11}$ where the lateral facet was found to have a length of $34.8 \pm 4.3 \mathrm{~mm}$ and the superoposterior facet to have a radius of $8.5 \pm 1.0 \mathrm{~mm}$ (diameter: $17 \mathrm{~mm}$ ). The values in the current study are smaller than those of the aforementioned paper because the methods of measuring the footprint were different. Robertson digitally analyzed the footprint around the most distal tendon portion, while the footprint length was outlined with a marker and were measured using a caliper. Futhermore, both facets were portrayed as squares in the current study. The lateral side may be smaller because both sides were measured as a square, so some portions that were lateral may be considered superoposterior. Additional anatomical data shows that tendon thickness was highly correlated to max load in the DR-KLA group $\left(\mathrm{R}^{2}=0.896\right.$, Figure 3.13).

The optical data shows there is a greater elongation on the lateral facet. Based on the statistical analysis of the optical data, it was found that at maximum load for both repairs the RTU on the lateral side demonstrated a large elongation. Clinically, this demonstrates that the lateral side is responsible for supporting most the load and undergoes a large amount of 
elongation due to its size. Thus, it would be desirable to fix the tendon over the original footprint as to not disturb these properties. Dividing the footprint into different facets may help improve the repair location, where surgeons will have a defined region to fit the tendon over the original footprint. Unfortunately, failure did not occur on the lateral side, instead most of the failures were on the superoposterior side. This could be an effect of the loading vector used in the experimental setup. Pulling the tendon under the prescribed loading conditions (Figure 2.10) may have caused the superoposterior side to undergo loads not normally applied.

The maximum load was strongly correlated to BMD in the DR-KLA group but not the DRMCS $\left(\mathrm{R}^{2}=0.816\right.$, Figure 3.12). Based on this analysis, BMD should be considered during surgical planning. This point is also conveyed in $\mathrm{Voos}_{\text {et }} \mathrm{al}^{15}$, in which suture anchors were placed into the footprint of the abductor tendons (gluteus medius and minimus) on the greater trochanter in a standard arthroscopic fashion. Both metal and bioabsorbable anchors were considered appropriate, but were only used depending on the bone quality. This may explain the anchor pullout of specimens with lower BMD at low loads. The DR-KLA would appear to be preferable due to strong fixation, technical ease, and time saving compared to the DR-MCS. Anchors did pull out of the bone at high loads for those with higher BMD. For this type of repair, anatomical data may be necessary when deciding on surgical intervention and will only strengthen the choice for this repair.

Both of the current repairs are Double Row techniques and have similar outcomes to those used in the rotator cuff. In the current literature, Double Row repairs and modified Double Row techniques have been thoroughly investigate, in which both are shown to be comparable 14,15,16,23,25. Mazzocca et $\mathrm{al}^{23}$ has reported that Double Row repairs (diamond, mattress double anchor, or modified mattress double anchor) have similar results at maximum load (all failed 
higher than $250 \mathrm{~N}$ ) and gap formation (cadaver model). Furthermore, Mahar et $\mathrm{al}^{24}$ found no significant biomechanical differences in terms of peak elongation and respective loads between two different Double Row repairs (bovine model). Both utilize the medial row stitch and suture anchors.

The recent use of suture anchors along with knotless anchors for soft tissue to bone fixation demonstrates a superior mechanical strength and both biomechanical and clinical success have been improved by the addition of a medial row complex. In the current study, both repair constructs utilize this configuration. By using anchors and the mattress stitch, there is significantly less motion between tendon and bone at repair site, application is quicker and easier, and the repairs have equivalent stability properties (knotless anchor vs sutures) ${ }^{24}$. Thus, the current results show both repairs have fixation properties that would be acceptable as with the rotator cuff. Further investigation is needed to see if more Double Row techniques have similar properties.

A limitation of this study is that the repair groups were tested in a cadaver model. As a result, effects of healing and rehabilitation following repair cannot be investigated and clinical outcomes of the repair cannot be determined. Other potential limitations of the current study were the limited number of intact specimens and the fact that these specimens were not evaluated for cyclic loading responses. Rather, intact specimens were used to obtain baseline measurements of the native tissue and to establish the cyclic testing protocol. Therefore, direct statistical comparisons of intact to repaired specimens' data are not warranted. Furthermore, in the Intact group, the insertion site location proved to be problematic because of extra tissue around the insertion and care was taken as to not damage the tendon. 
The setup used limits the study to only testing in one direction. Since the GM muscle is responsible for abduction and rotation, the fibers may move in a different direction and failure may occur by one of these actions. This was not able to be replicated. Furthermore, a single camera was used which could only output x- and y-directions. During loading, it would have been beneficial to obtain any other motion of the tendon-muscle or repair-tendon contruct to show the path of failure.

The current study is among the first biomechanical investigations of the gluteus medius muscle-tendon-bone construct. Furthermore, the current study demonstrates good initial fixation stability properties for both repairs. It is advised that repair techniques are decided based on a patient specific rationale. When repairing, the exact reattachment to the footprint is essential in order to establish the correct fixation and functionality of the tendon-bone unit. Anatomical data from patients are vital to the repair method. BMD must be taken into account because anchors, while strong and able to handle higher loads, may fail due to poor bone quality. Both repairs demonstrate adequate fixation properties and suture retention during the cyclic loading and load to failure tests. There were differences found between the repairs, but similarities were shown as well. Therefore, the DR-KLA and DR-MCS repairs would be acceptable methods to use for correcting complete GM tears. Both clinical and mechanical outcomes are supportive towards this repair. 


\section{CITED LITERATURE}

1. Functional Anatomy of the Hip Joint. 2009 June:237-50<https://catalog.amaassn.org/MEDIA/ProductCatalog/m890153/\%20Function\%20\%20Anatomy\%20Ch\%207.pdf>

2. Robertson WJ, Gardner MJ, Barker JU, Boraiah S, Lorich DG, Kelly BT. Anatomy and dimensions of the gluteus medius tendon insertion. Arthroscopy. 2008 Feb;24(2):130-6.

3. Strauss EJ, Nho SJ, Kelly BT. Greater trochanteric pain syndrome. Sports Med Arthrosc. 2010 Jun;18(2):113-9.

4. Kingzett-Taylor A, Tirman PF, Feller J, McGann W, Prieto V, Wischer T, Cameron JA, Cvitanic O, Genant HK. Tendinosis and tears of gluteus medius and minimus muscles as a cause of hip pain: MR imaging findings. AJR Am J Roentgenol. 1999 Oct;173(4):1123-6.

5. Ozçakar L, Erol O, Kaymak B, Aydemir N. An underdiagnosed hip pathology: apropos of two cases with gluteus medius tendon tears. Clin Rheumatol. 2004 Oct;23(5):464-6.

6. Connell DA, Bass C, Sykes CA, Young D, Edwards E. Sonographic evaluation of gluteus medius and minimus tendinopathy. Eur Radiol. 2003 Jun;13(6):1339-47.

7. Voos JE, Shindle MK, Pruett A, Asnis PD, Kelly BT. Endoscopic repair of gluteus medius tendon tears of the hip. Am J Sports Med. 2009 Apr;37(4):743-7.

8. Bunker TD, Esler CN, Leach WJ. Rotator-cuff tear of the hip. J Bone Joint Surg Br. 1997 Jul 79(4):618-20.

9. Bunker TD. Tendon disorders of the shoulder and elbow. Current opinion in orthopaedics. 1995;7;IV:68-72.

10. Miozzari HH, Dora C, Clark JM, Nötzli HP. 10. Late repair of abductor avulsion after the transgluteal approach for hip arthroplasty. J Arthroplasty. 2010 Apr;25(3):450-457.

11. Howell GE, Biggs RE, Bourne RB. Prevalence of abductor mechanism tears of the hips in patients with osteoarthritis. J Arthroplasty 2001;16:121-123. 
12. Benjamin M, Kaiser E, Milz S. Structure-function relationships in tendons: A review. J Anat. 2008 Mar;212(3):211-28.

13. Rumian AP, Wallace AL, Birch HL. Tendons and ligaments are anatomically distinct but overlap in molecular and morphological features--a comparative study in an ovine model. J Orthop Res. 2007 Apr;25(4):458-64.

14. Lorbach O, Bachelier F, Vees J, Kohn D, Pape D. Cyclic loading of rotator cuff reconstructions: single-row repair with modified suture configurations versus double-row repair. Am J Sports Med. 2008 Aug;36(8):1504-10.

15. Ma CB, MacGillivray JD, Clabeaux J, Lee S, Otis JC. Biomechanical evaluation of arthroscopic rotator cuff stitches. J Bone Joint Surg Am. 2004 Jun;86-A(6):1211-6.

16. MacGillivray JD, Ma CB. An arthroscopic stitch for massive rotator cuff tears: the Mac stitch. Arthroscopy. 2004 Jul;20(6):669-71.

17. Wang V, Wang FC, McNickle A, Friel N, Yanke A, Chubinskaya S, Romeo A, Verma N, Cole B. Medial Versus Lateral Supraspinatus Tendon Properties : Implications for Double-Row Rotator Cuff Repair. Am J Sports Med 2010 38: 2456.

18. Nho S, Frank R, Van Thiel G, Wang FC, Wang V, Provencher M, Verma N. A Biomechanical Analysis of Shoulder Stabilization : Posteroinferior Glenohumeral Capsular Plication. Am J Sports Med 2010 38: 1413.

19. Gupta A, Lattermann C, Busam M, Riff A, Bach, Jr B, Wang V. Biomechanical Evaluation of Bioabsorbable Versus Metallic Screws for Posterior Cruciate Ligament Inlay Graft Fixation : A Comparative Study. Am J Sports Med 2009 37: 748. 
20. Van Thiel G, Wang V, Wang FC, Nho S, Piasecki D, Bach Jr. B, Romeo A. Biomechanical similarities among subscapularis repairs after shoulder arthroplasty. J Shoulder Elbow Surg (2010) 19, 657-663.

21. Voos JE, Rudzki JR, Shindle MK, Martin H, Kelly BT. Arthroscopic Anatomy and Surgical Techniques for Peritrochanteric Space Disorders in the Hip. Arthroscopy. 2007 Nov;23(11):1246.e1-5.

22. Shindle M, Voos J, Heyworth B, Mintz D, Moya L, Buly R, Kelly B. Hip Arthroscopy in the Athletic Patient: Current Techniques and Spectrum of Disease. J Bone Joint Surg Am. 2007;89:29-43.

23. Wall L, Keener J, Brophy R. Double-row vs single-row rotator cuff repair: A review of the biomechanical evidence. J Shoulder Elbow Surg (2009) 18, 933-941.

24. Pauly S, Gerhardt C, Chen J, Scheibel M. Single versus double-row repair of the rotator cuff. Does double-row repair with improved anatomical and biomechanical characteristics lead to better clinical outcome? Knee Surg Sports Traumatol Arthrosc (2010) 18:1718-1729.

25. Milano G, Grasso A, Zarelli D, Deriu L, Cillo M, Fabbriciani C. Comparison between singlerow and double-row rotator cuff repair: a biomechanical study. Knee Surg Sports Traumatol Arthrosc (2008) 16:75-80.

26. Bigliani L, Pollock R, Soslowsky L, Flatow E, Pawluk R, Mow V. Tensile Properties of the Inferior Glenohumeral Ligament. Journal of Orthopaedic Research (1992) 10:187-197.

27. Danto M, Woo S. The Mechanical Properties of Skeletally Mature Rabbit Anterior Cruciate Ligament and Patellar Tendon over a Range of Strain Rates. Journal of Orthopaedic Research (1993) 11:58-6. 
28. Elliot D, Robinson P, Gimbel J, Sarver J, Abboud J, Iozzo R, Soslowski L. Effect of Altered

Matrix Proteins on Quasilinear Viscoelastic Properties in Transgenic Mouse Tail Tendons.

Annals of Biomedical Engineering (2003) 31:599-605.

\section{Appendix/Standard Operating Protocol (SOP)}

CT-Bone Mineral Density Protocol

Contact for scheduling CT scans: Kerry Kraushaar KKraushaar@rushortho.com

-Positioning

1) When CT scanning the specimen, the whole hemipelvis should be intact. The specimen should be still frozen and wrapped.

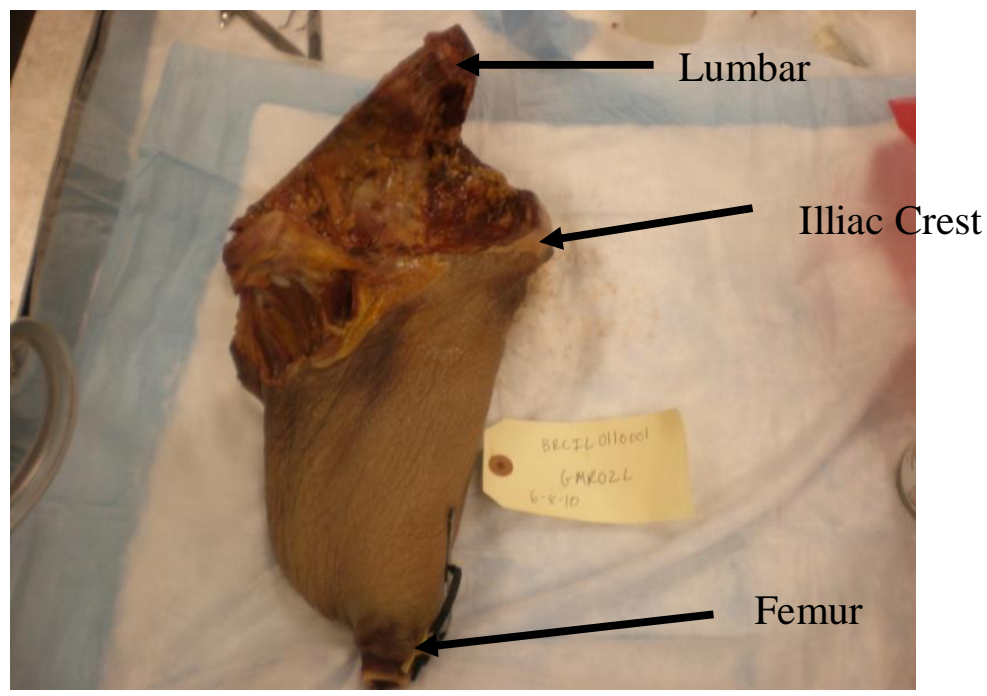

2) Place the hemipelvis on the CT bed- use lower extremity on computer and name it specifically to study to find in MedView.

3) The position will be similar to a patient lying on their back, in which their head goes through the scan first.

4) In order to see the femoral head, the specimen was angled medially as to ensure that the iliac crest does not show up over the femoral head or greater trochanter. Sand bags are used to hold the specimen in place ( $3 \mathrm{~mm}$ slices, $0.5 \mathrm{~mm}$ pixel size, $120 \mathrm{KV}$ ). 


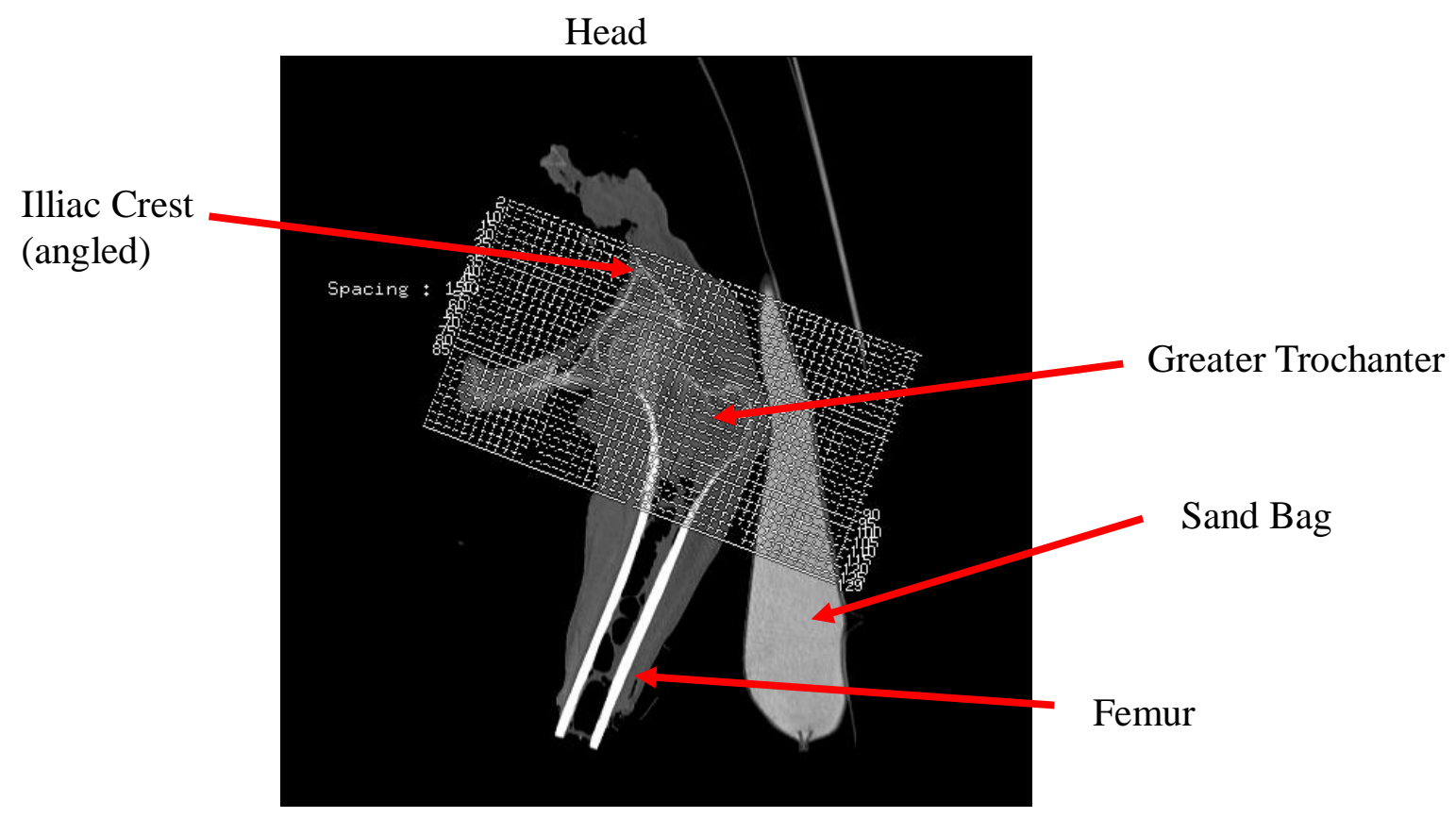

Feet

\section{-Calculations}

Using axial view move to the position where the greater trochanter is most visible.

1) Select Measure density in rectangle tool in Mimics measurement menu.

This tool allows you to measure the area and average density within a rectangular region. Move the rectangle to the image you want to measure. Release the mouse button when you are satisfied with the size of the rectangle (approximately $30 \mathrm{~mm}^{2}$, as to reduce variation from cortical bone and degeneration patches). You can adjust the size of the rectangle by dragging a border or corner when the cursor changes to a white double arrow. To move the rectangle, place the cursor on a yellow border (the cursor changes to a hand) and drag it. Try to locate it within the trabecular bone region (see figure below) and so no cortical bone is touching.

Information appears about the area $\left(\mathrm{mm}^{2}\right)$, the mean density (Hounsfield Units) and the standard deviation of the density (Hounsfield Units) within the rectangle. The measurement data is recalculated when you release the mouse button.

2) Record the measurements (area, the mean density and the standard deviation of the density).

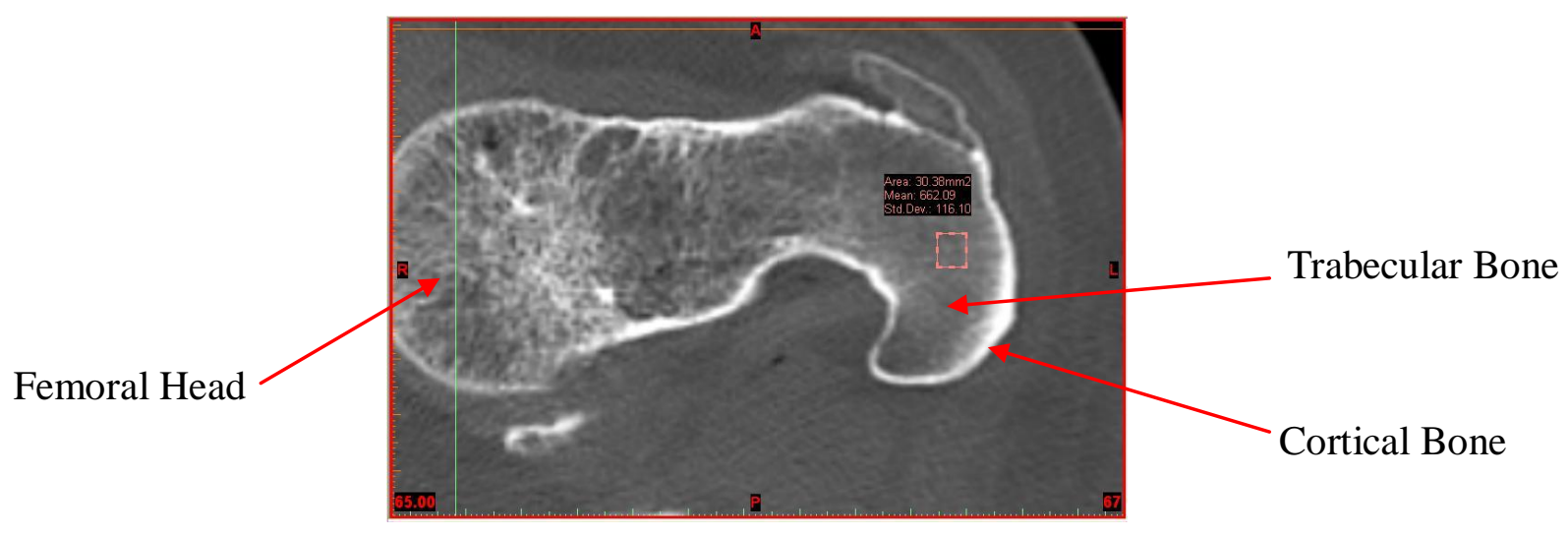


3) Scroll up (cranial direction) one slide (about 3mm). The measurements are updated on each slide. Make sure cortical shell is not within rectangle. The measurement box should stay in position, so there is no need to change the box location.

4) Record the measurements (area, the mean density and the standard deviation of the density) from the second slide.

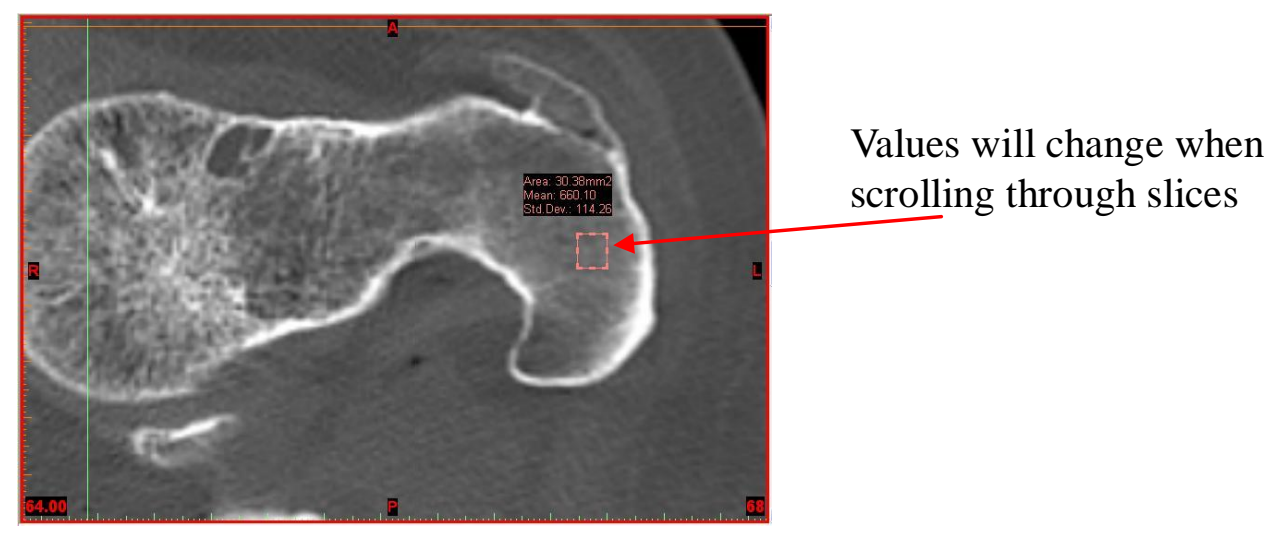

5) Scroll up (cranial direction) one slide. The measurements are updated on each slide. Make sure cortical shell is not within rectangle.

6) Record the measurements (area, the mean density and the standard deviation of the density) from the third slide.

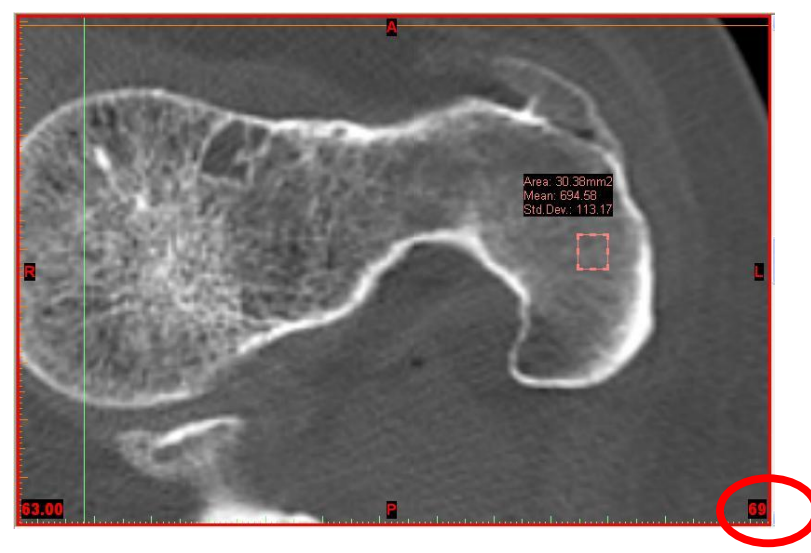

Slice Number

7) Average the three densities for a given specimen.

8) Repeat steps 1-7 for the rest of the specimens. 
Testing \& Data Protocol

Digital Camera- video capture of markers on tissue

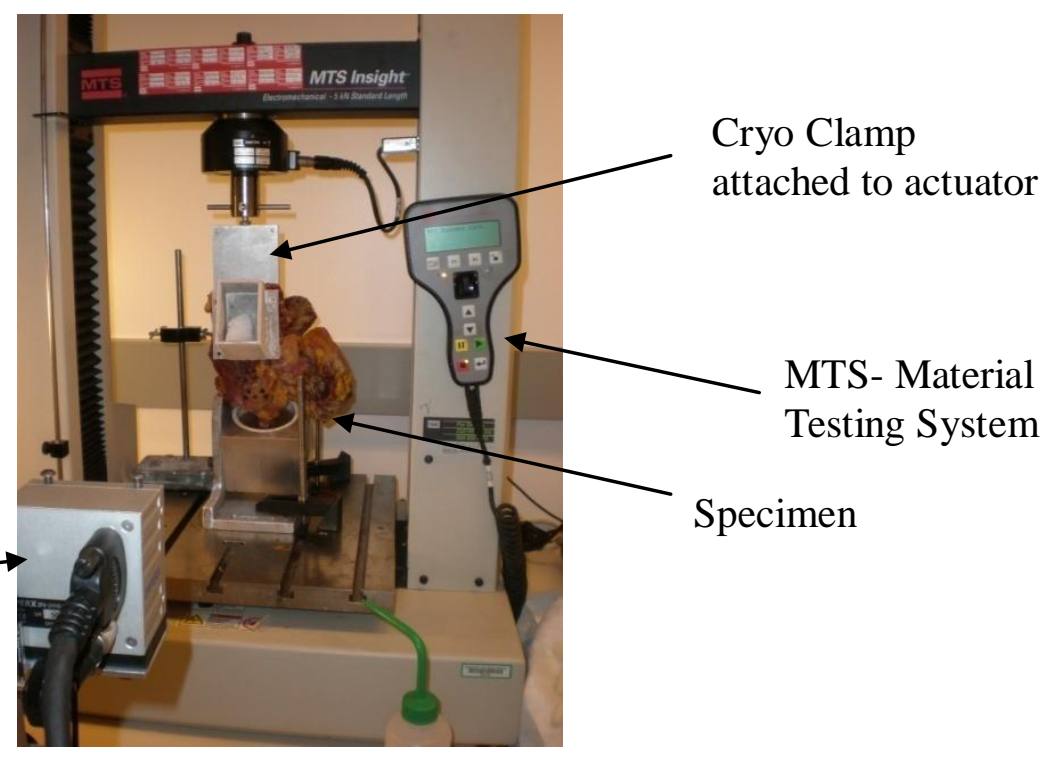

Camera: Imperx IPX-1M48-L, 1000x1000x48 fps

Camera Lens: $25 \mathrm{~mm}$

Camera to MTS: $\sim 60 \mathrm{~cm}(23.5 \mathrm{in})$

Marker Size: $3 / 32$ in

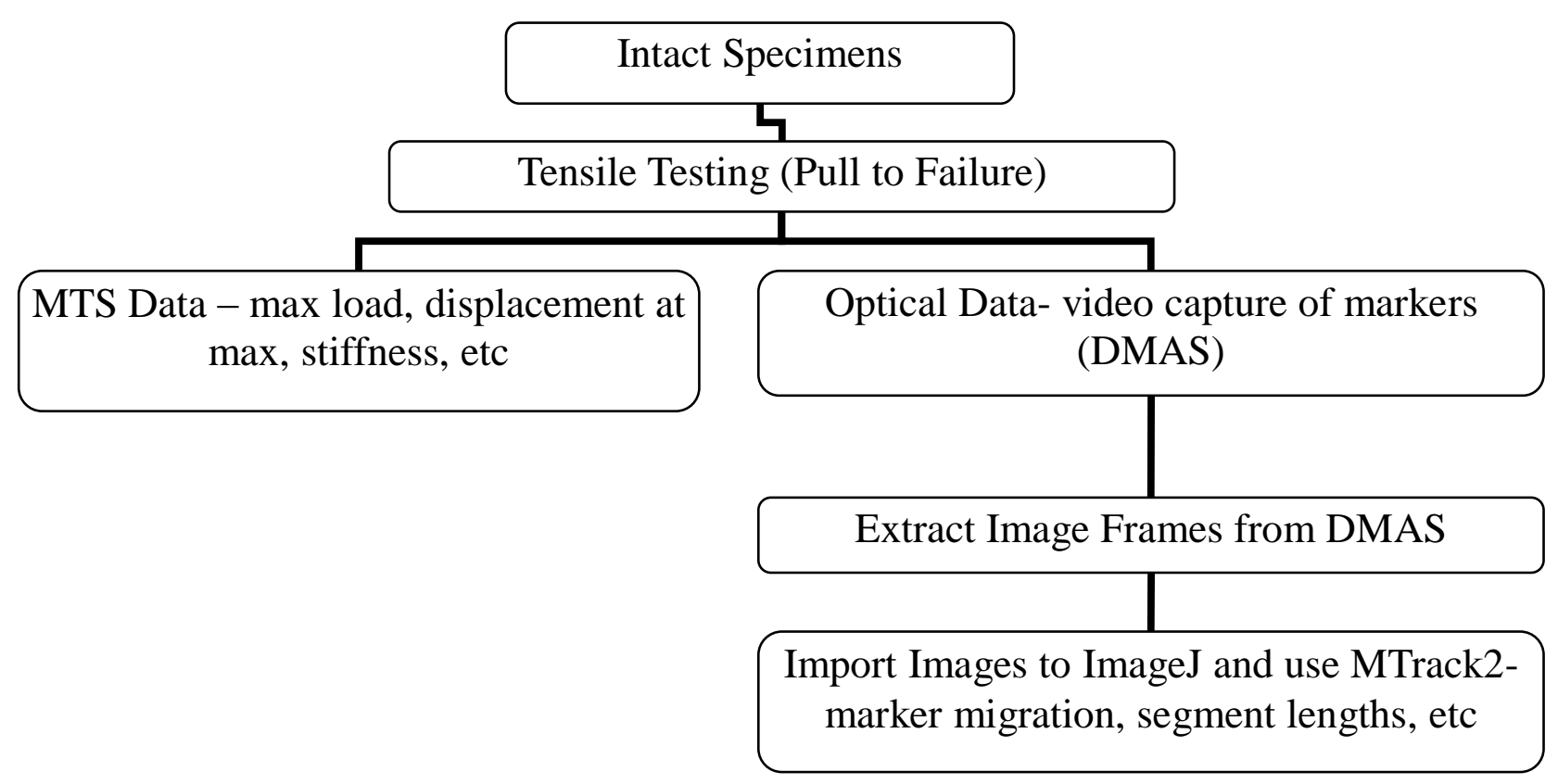




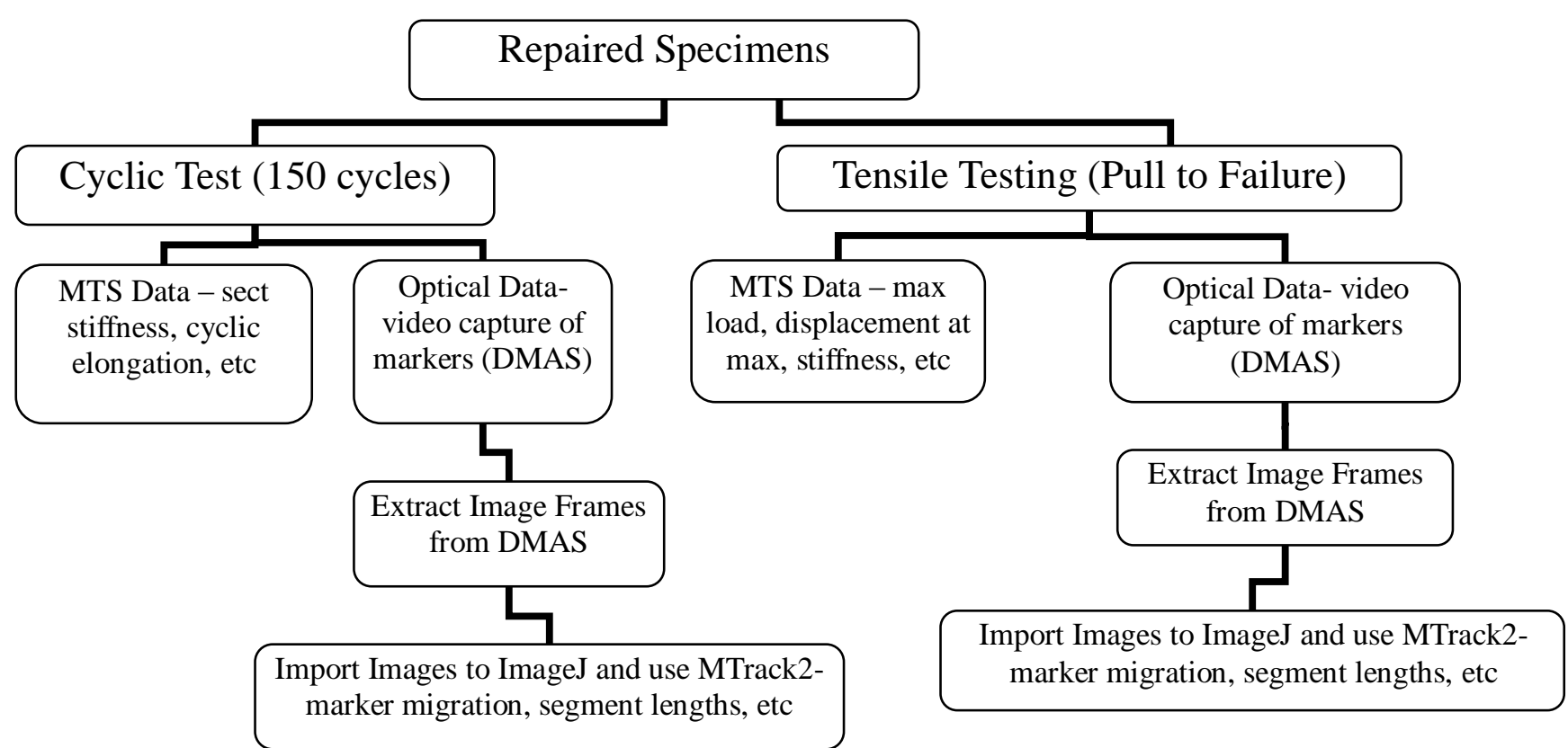

\section{ImageJ MTrack2 Protocol}

Step1- DMAS Image Extraction

- In order to get the actual images to analyze, each frame must be extracted from DMAS. Open DMAS Recorder and open a test file.

- The title of the test should display under Datasets. Go to Video Sources and click to open the arrow. Underneath, SRC001 will dsiplay. Right click on it and you will be give the option to Export to AVI or Export to Image Sequence. Click on Export to Image Sequence

- The program will ask you where you would like to save the images. Give the folder an appropriate name. Click Save. This proccess may take some time because you will end up with more then 15000 frames (+-). 


\section{Step2- Start Up}

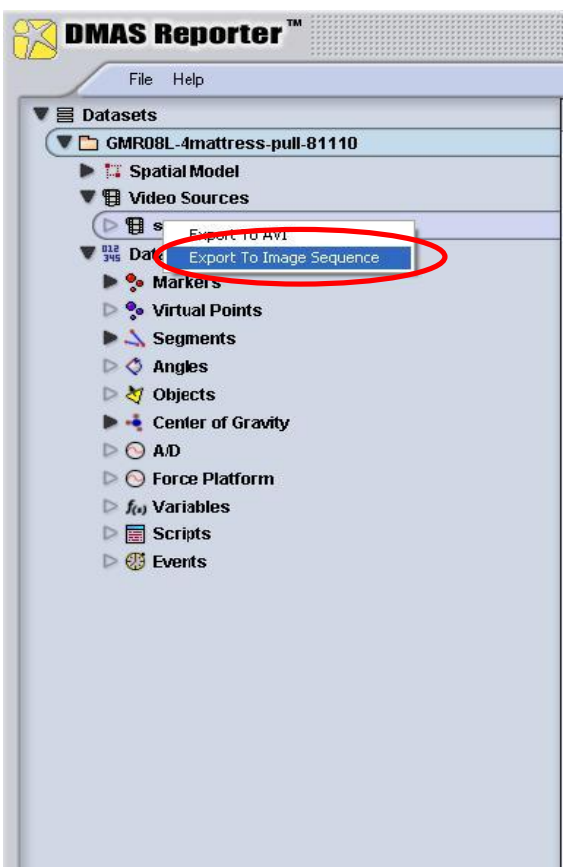

- Open Image $\mathbf{J}$

- Under Plugins, check to make sure that the MTrack2 program is saved and visble. If it is not, check where the program was saved or rerun Image $\mathbf{J}$

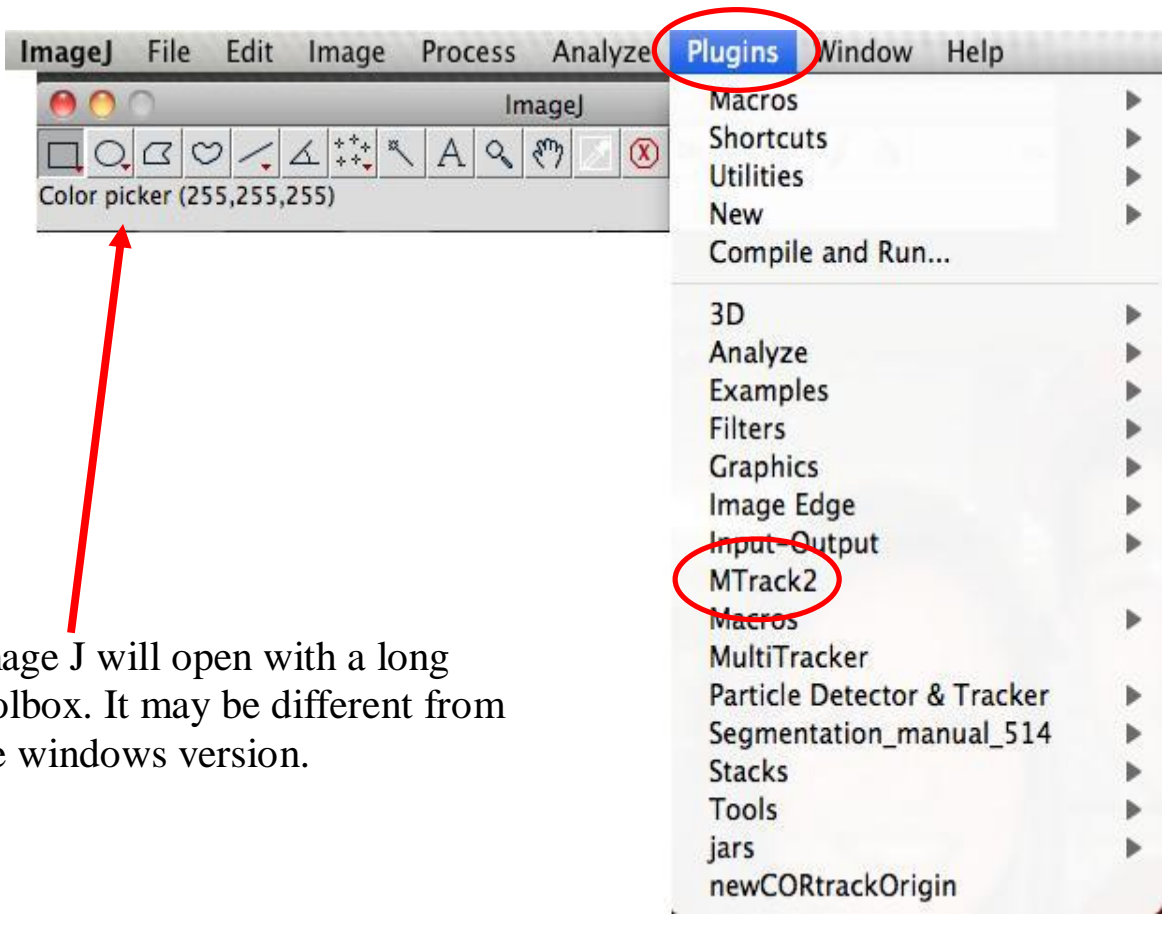


Step3- Importing and Memory

- Under File, there will be an option to Import the Image Sequence. The image sequence should be the selected frames chosen to be analyzed. The frames can contain the beginning of the failure test to maximum load or the first five cycles of the cyclic test. Make sure that the first frame is one of the specimen and NOT the black and white noise before the trigger turns on.

- After choosing the $1^{\text {st }}$ frame, a dialogue box will display with the number of frames and other options. Make sure the total number of frames is correct and convert to 8-bit Grayscale

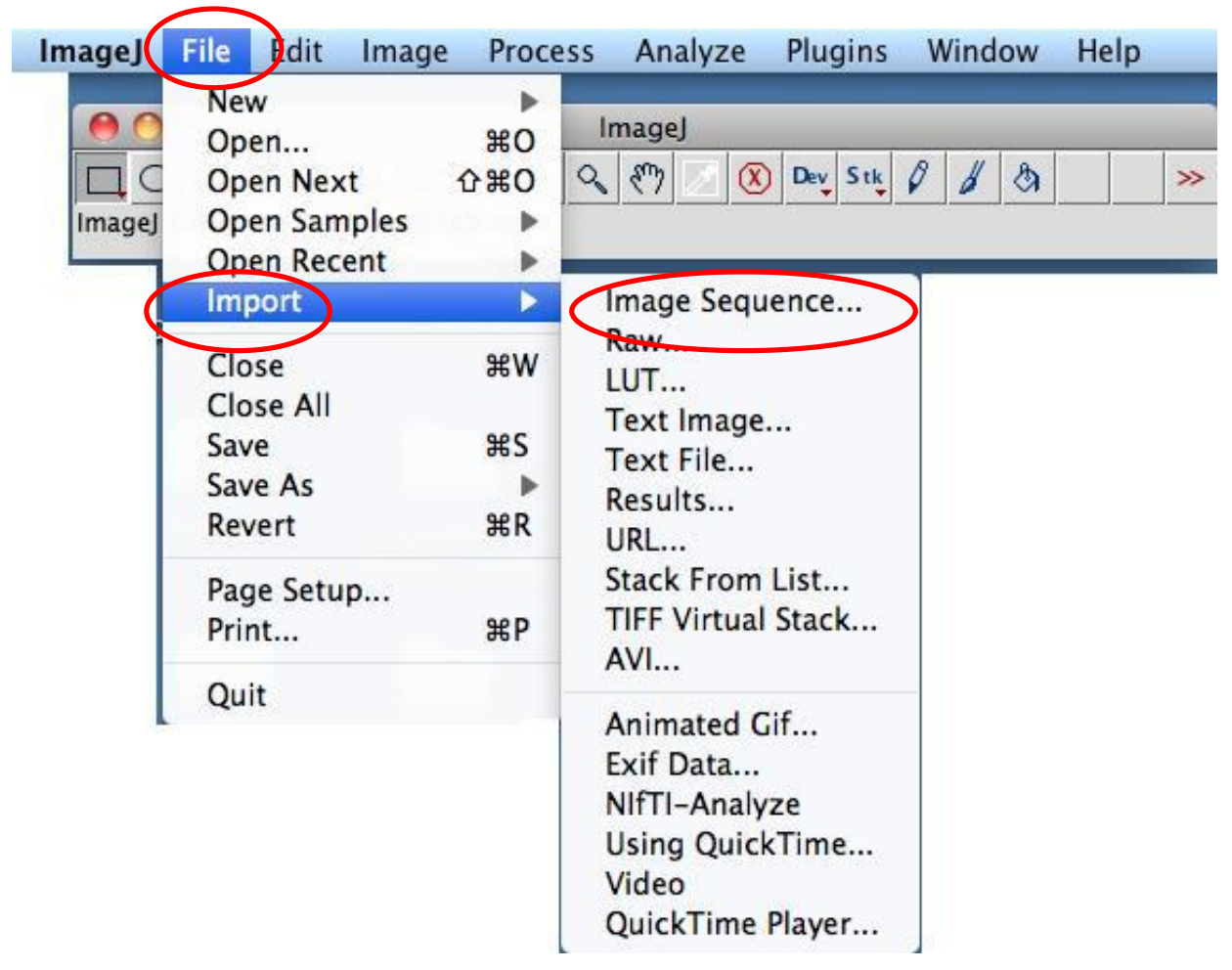



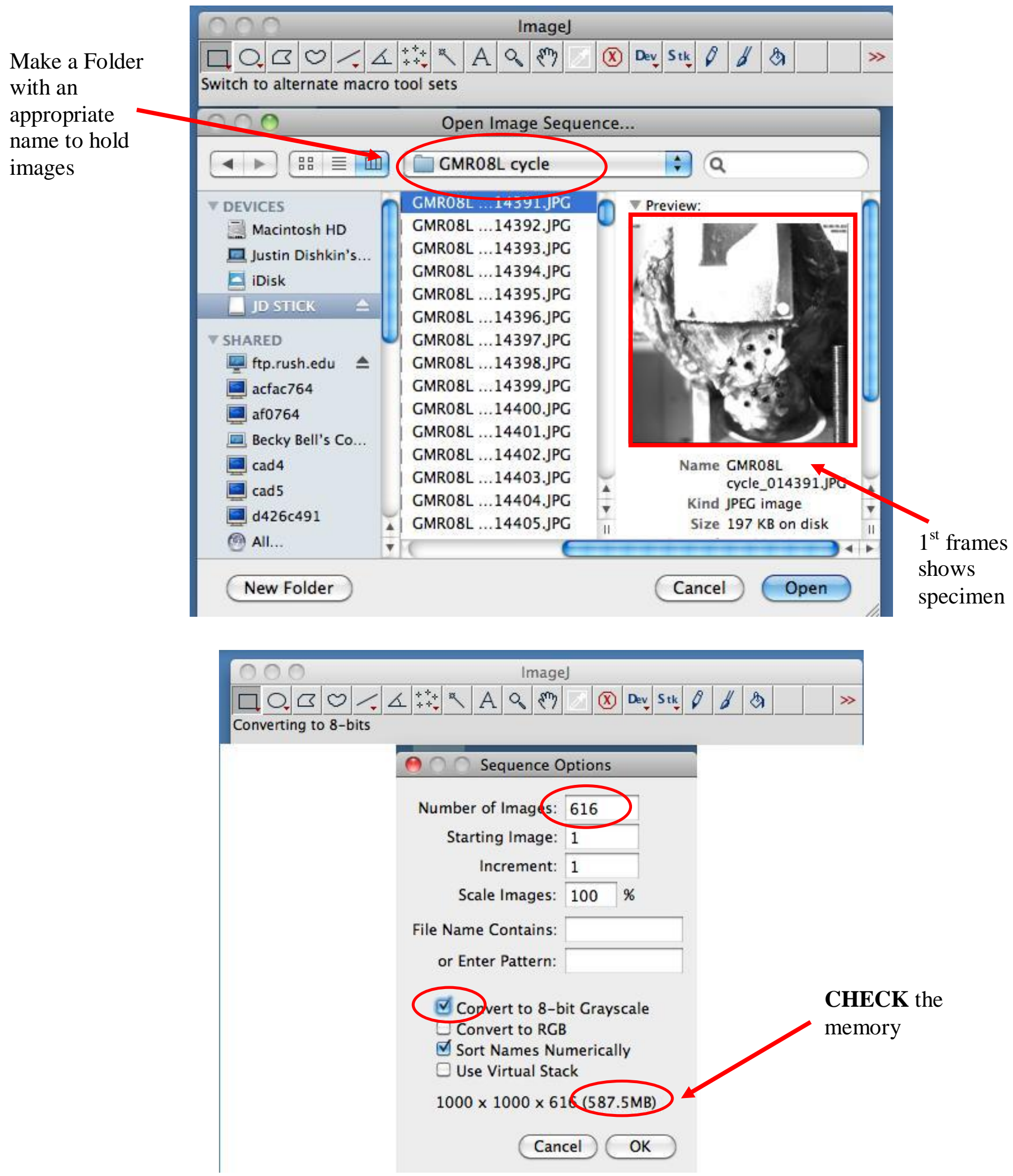
- Image $\mathbf{J}$ has a default memory setting that may be too low for some applications. There is a limit to how high the memory can go- $1700 \mathrm{MB}$. In order to change this, go to Edit, then Options, and then Memory and Threads. A dialogue box will display where you can type in the memory desired.

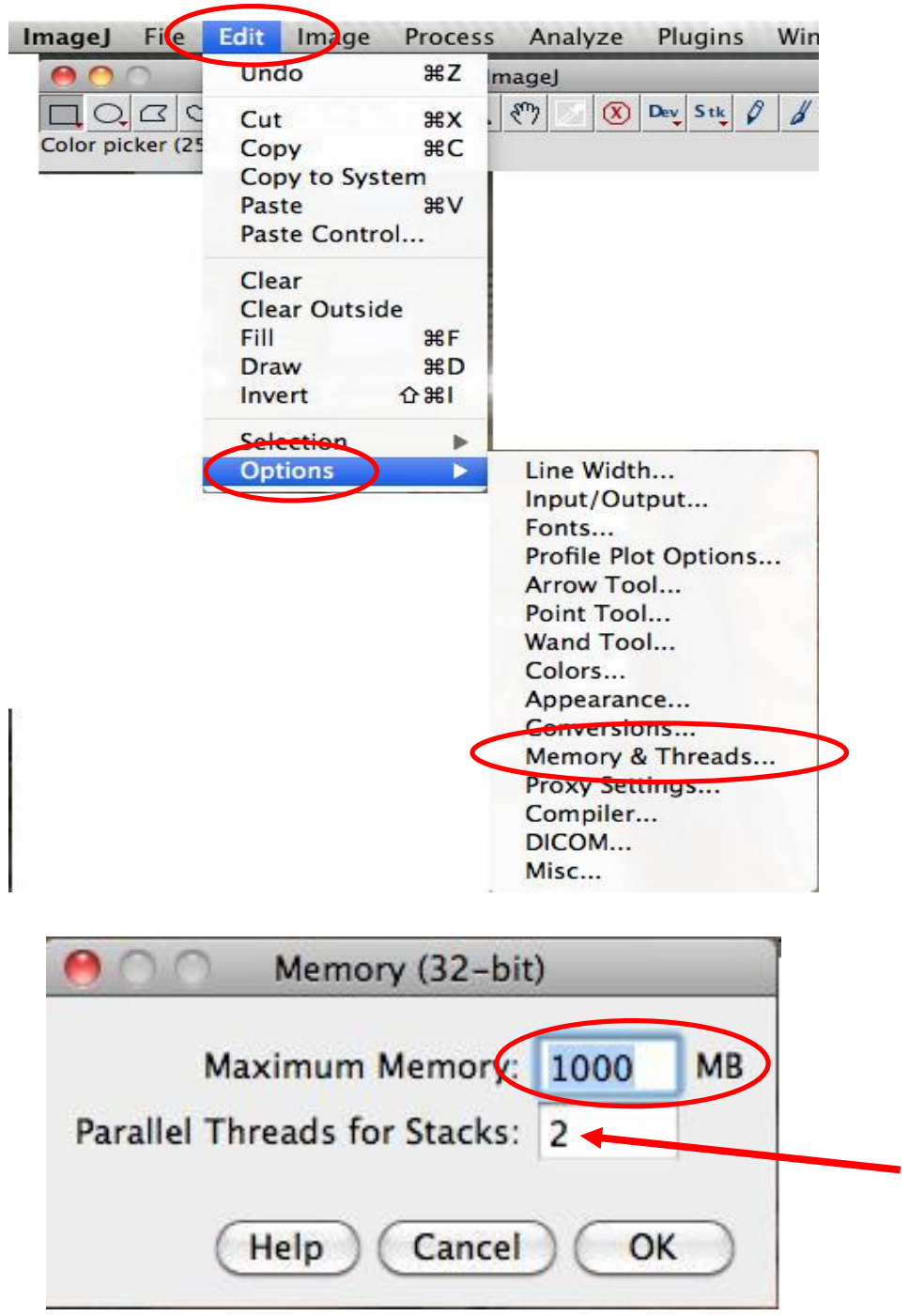

Don't need to change the second option. Make sure memory will incorporate all frames needed

Step4- Threshold and Crop

- The frames will be displayed and compiled in one box. The scroll button on the bottom allows you to see each frame along with an option to play it as a move.

- Similar to the DMAS tracking, you will need to threshold the images. Under Image, go to Adjust, and then Threshold

- A separate dialogue box will display. Use the bottom adjuster to lighten the image to make the markers more pronounced. The number will vary from specimen to specimen. You will be able to see the change as you adjust the 
contrast. When satisfied, press Apply and the thresholding will be set for ALL frames.

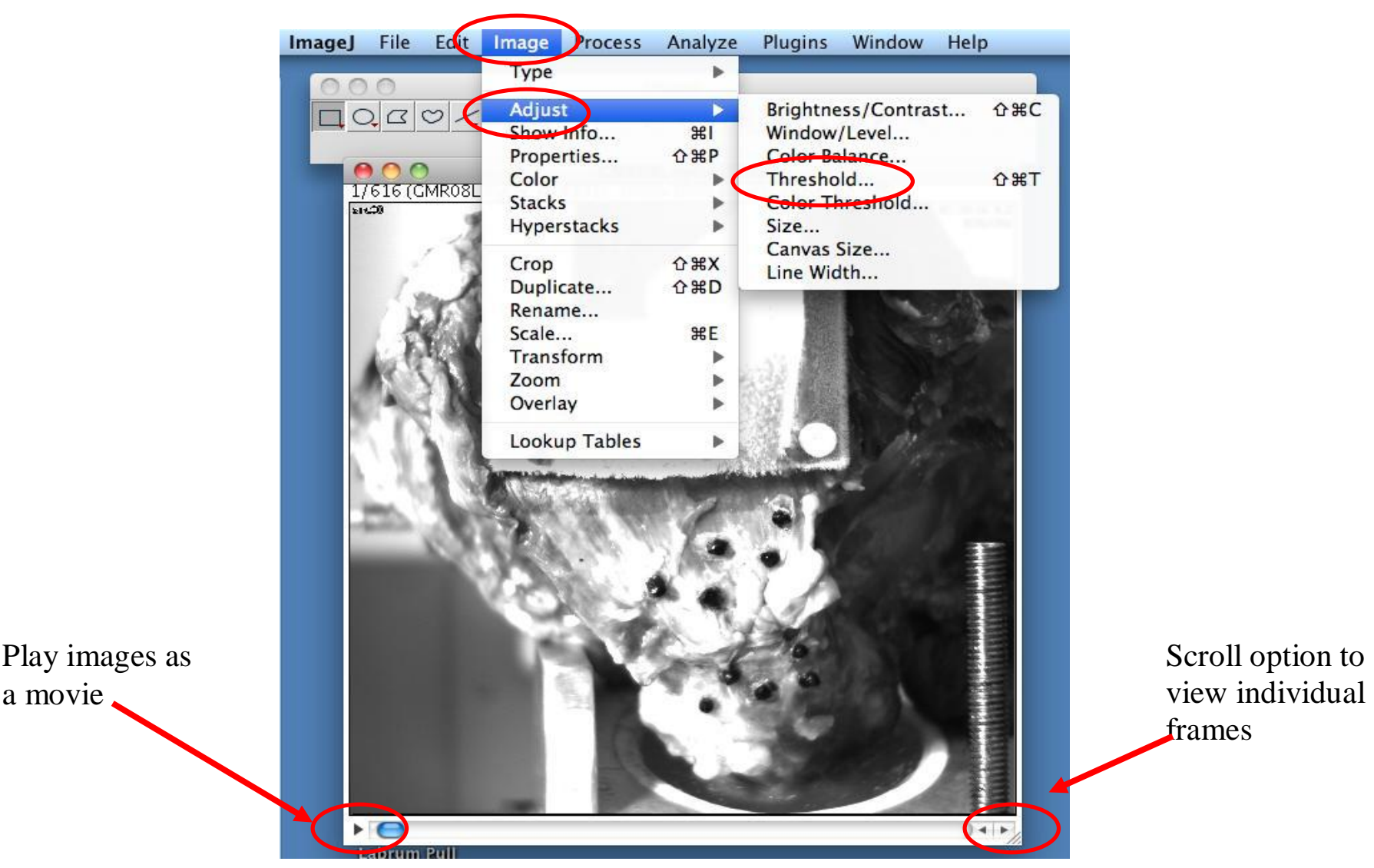




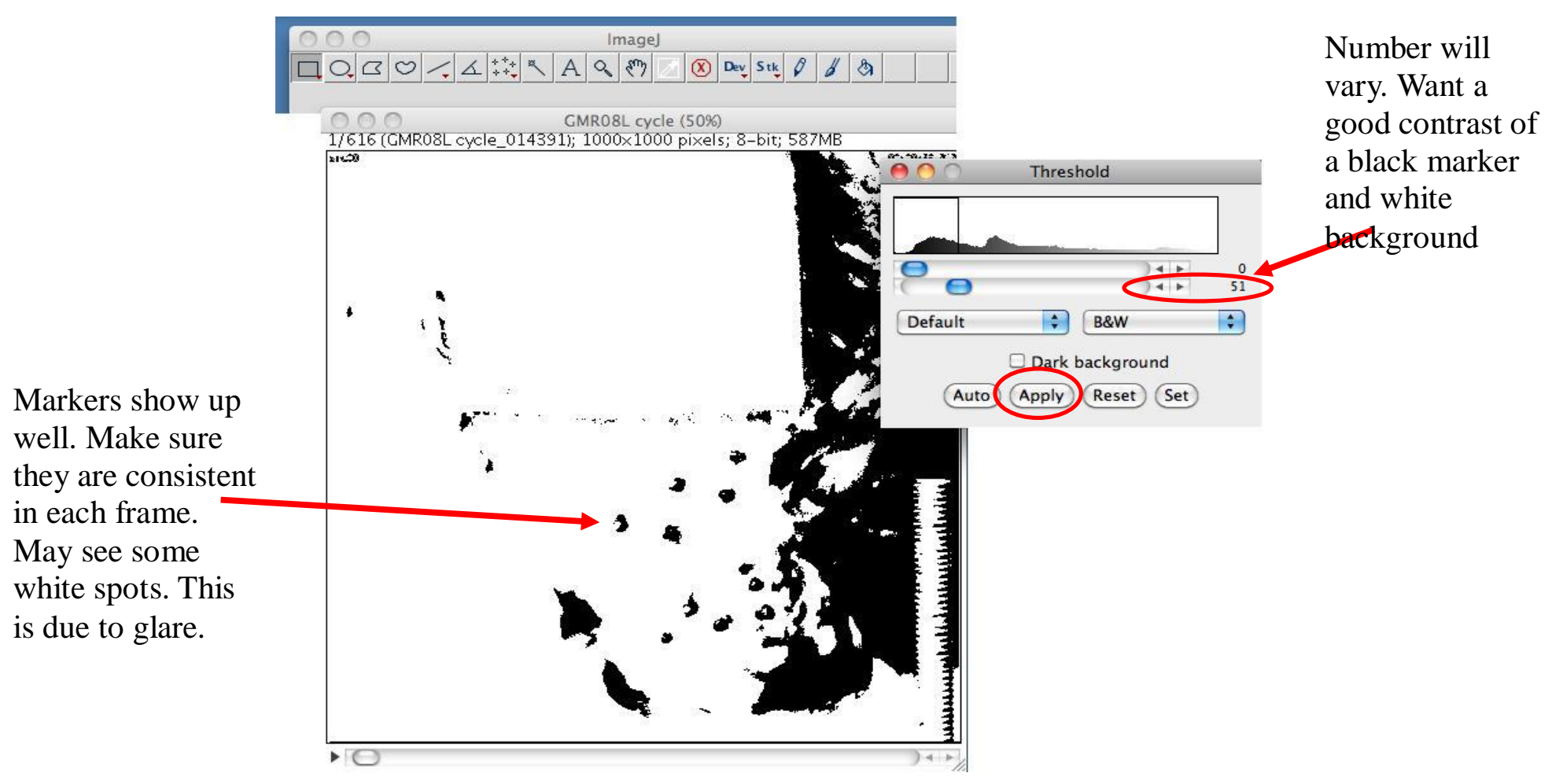

- After thresholding, the markers should be pretty isolated. In order to minimize the amount of noise, the images can be cropped. This will allow the program to focus on a region of interest (markers).

- Select the Box tool from the Image J options. Draw a box around the desired markers. Scroll to the last frame and make sure all the markers are incorporated in the drawn box.

- When satisfied, under Image, select Crop. A new box will display your cropped images. All frames are now cropped with your specific area. 

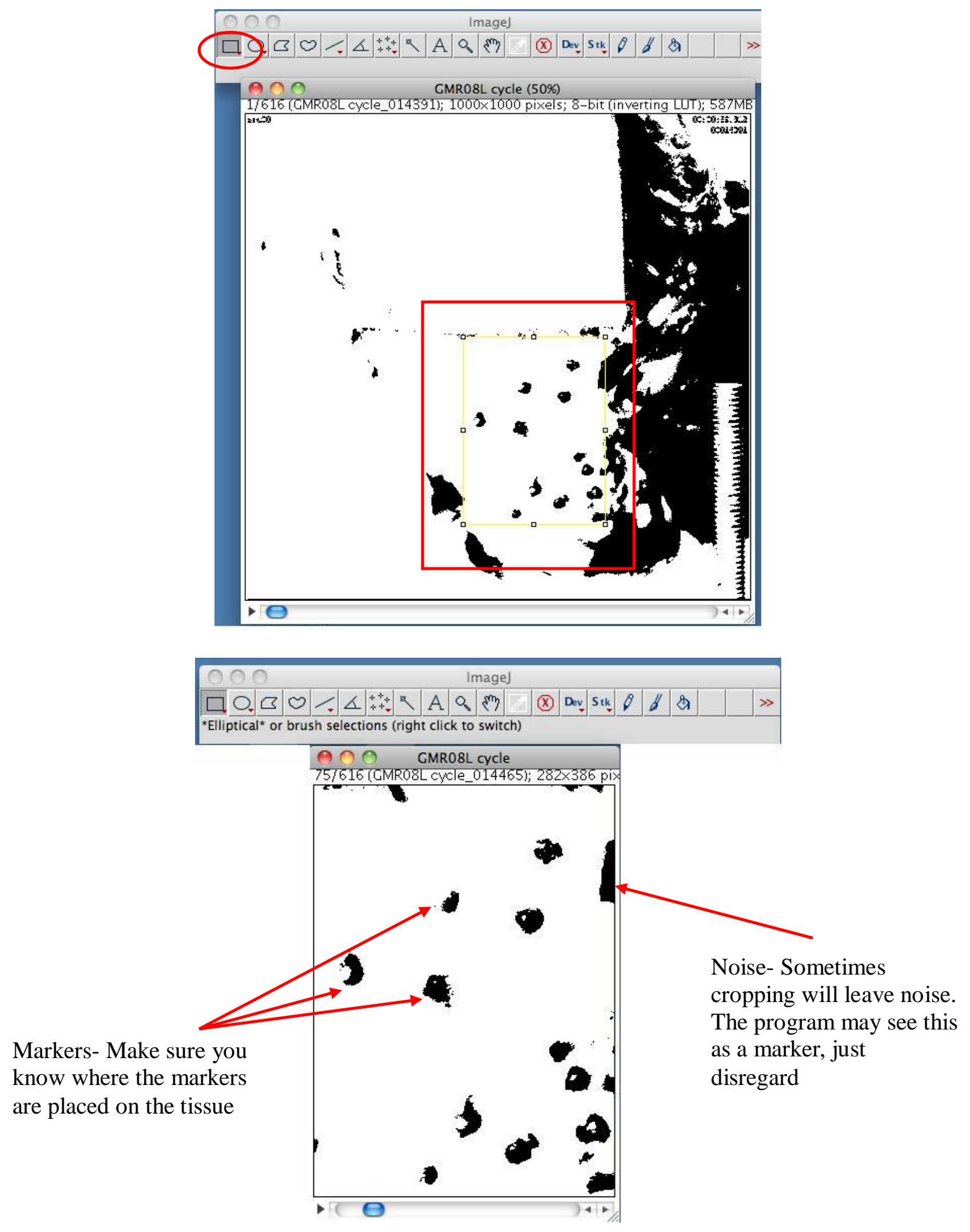
Step5- Analyzing

- To execute the tracking analysis, you will need to select the MTrack2 plugin. Under Plugins, go to MTrack2. This will bring up another dialogue box.

- The option in the dialogue box will represent how the program sees the markers and how the results will be displayed.

- Minimum and Maximum Object Size: Trail and error works best for these. The wider the range the better. These will take into account the pixel area of the markers and noise. Sometimes the markers will not be recognized until a few frames into the test.

- Maximum velocity: The more frames that are incorporated the better. This way the velocity will be rather lower and the markers will be consistent throughout the video

- Minimum Track Length: Input the number of frames loaded.

- After you have selected your inputs, check the boxes Display Path Length, Show Labels, and Show Position. Press OK.

- The program will begin analyzing and two additional windows will display. One contains the coordinates $(\mathrm{x}, \mathrm{y})$ for each marker found for all the frames. Also, at the bottom of the result table are calculated distances and lengths of the markers. The second window contains a similar image to the copped image, but has labels where the program believes there are markers.

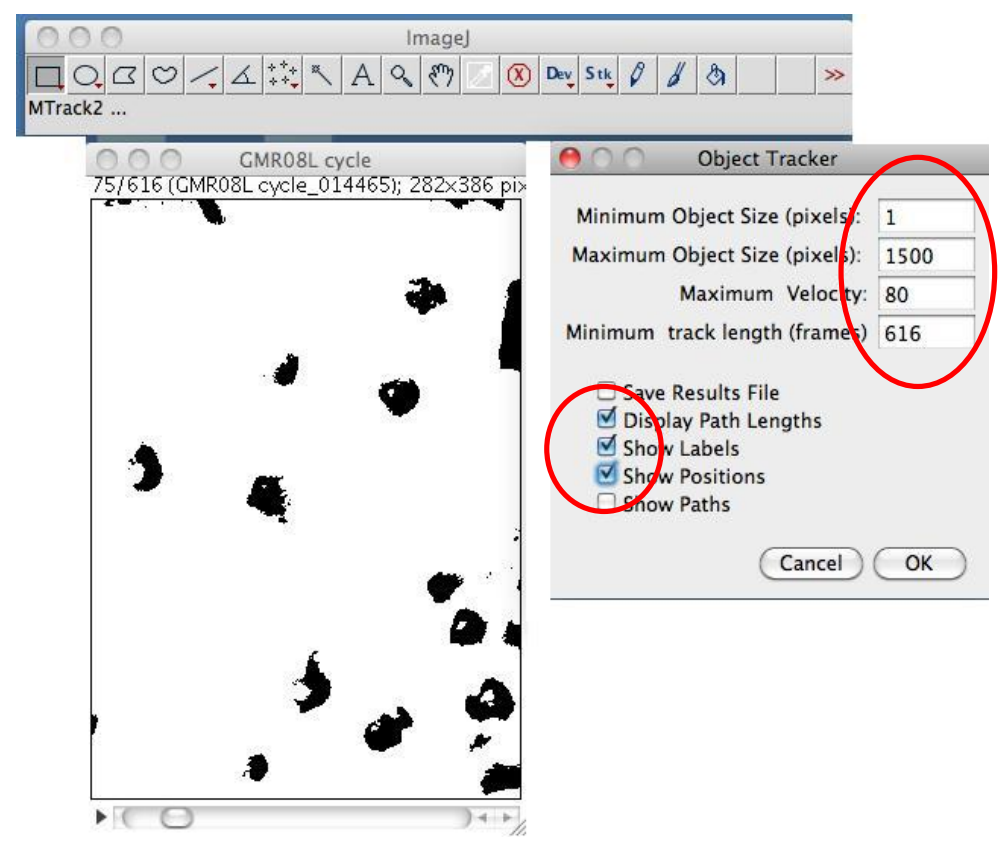




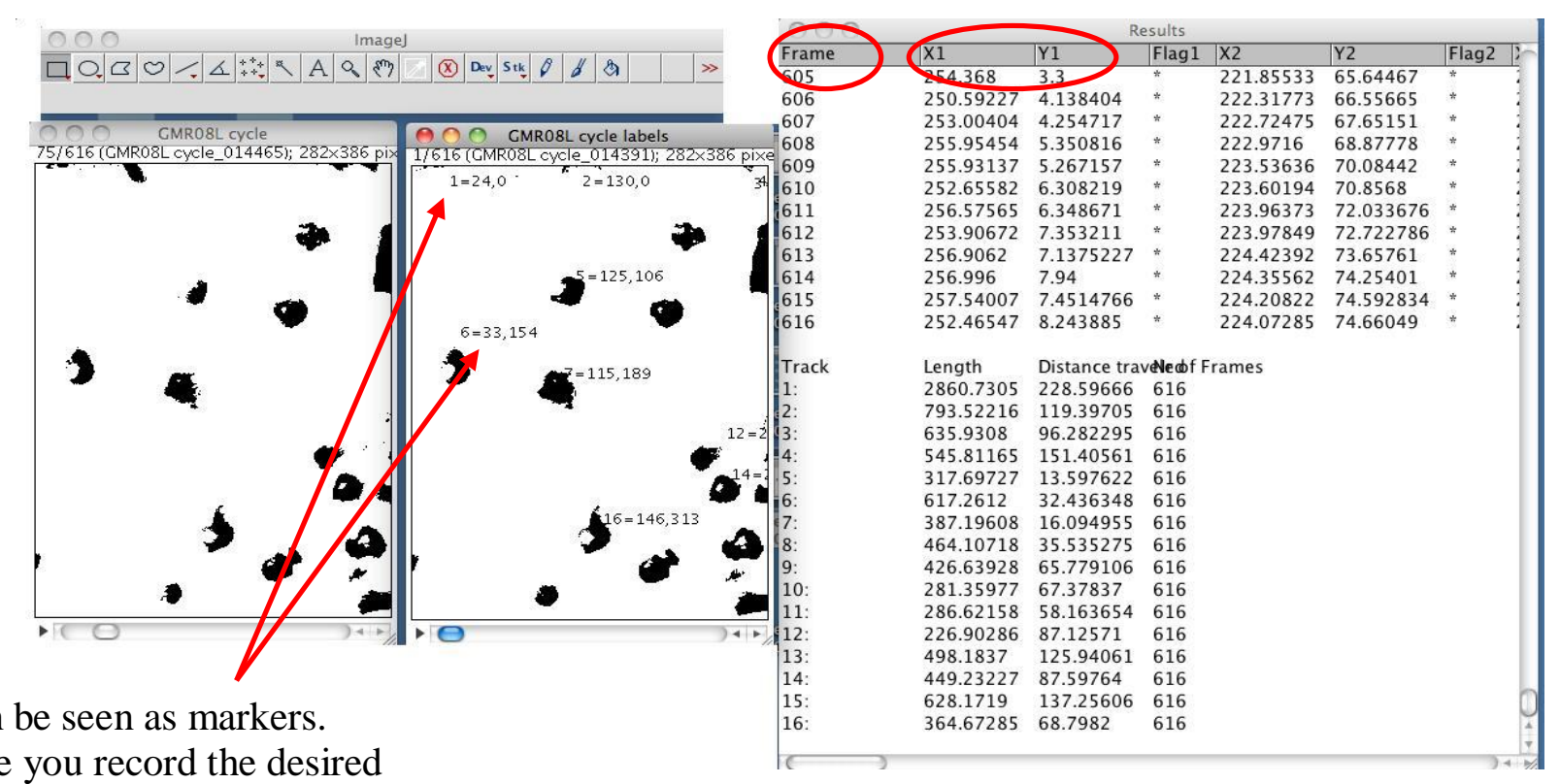

Noise can be seen as markers.

Make sure you record the desired

labels that match your markers

- Scroll through the entire video to make sure the markers are labeled and that the labels stay with the same maker. Sometimes, the labels will switch between markers or stay with a certain marker and disappear.

- Once satisfied, close out of the results table and labeled marker windows. Rerun MTrack2. Make sure your numbers are the same and check Save Results File.

- Choose an appropriate place to save the file. Give the file an appropriate name. Change the file extension to .xls. This will allow you to open the results in Excel for any calculations needed.

- If necessary, the labeled markers can be saved as well. Under File, go to Save As, and the frames will be saved together as a tiff file. 


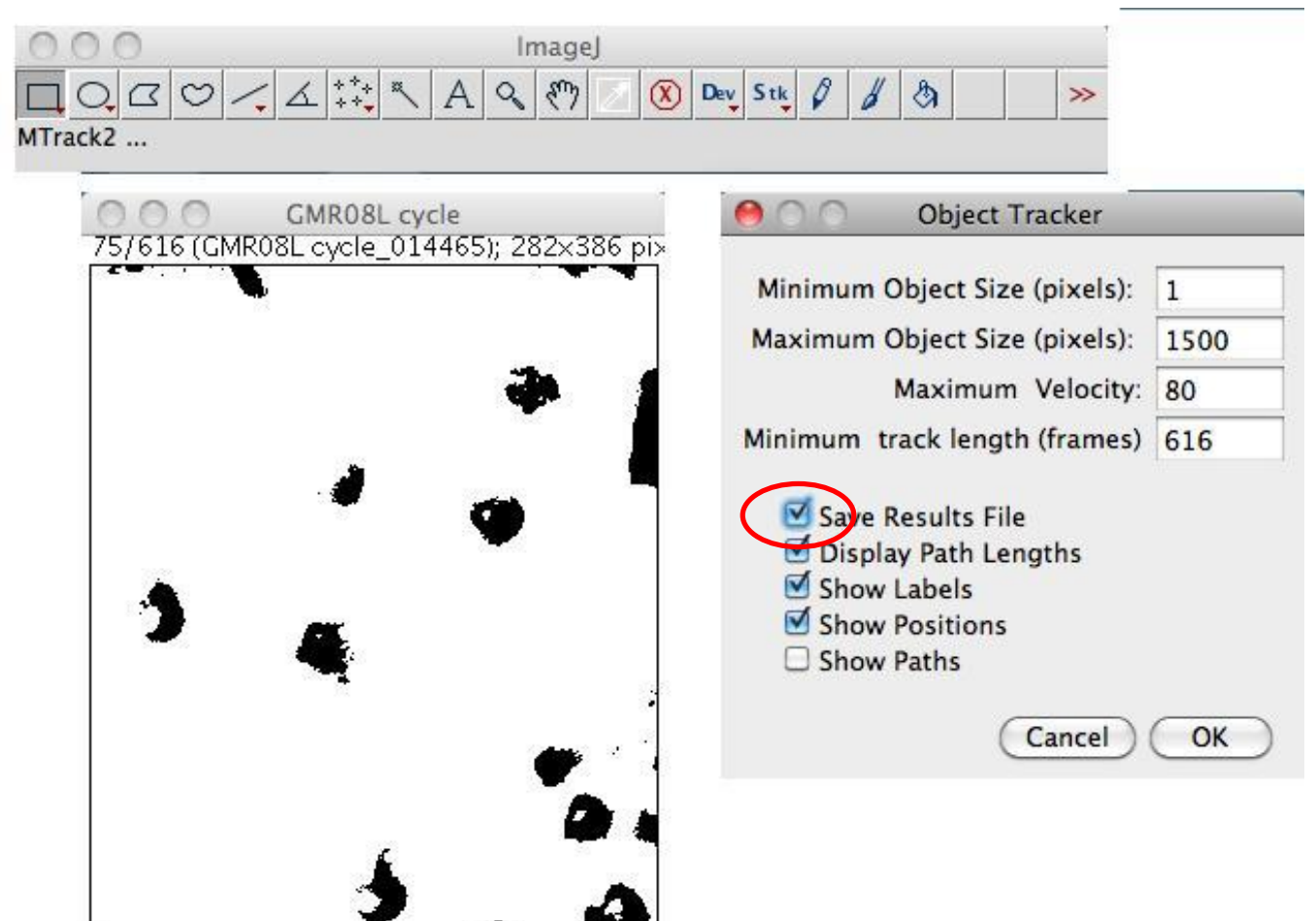

Make sure you save the cyclic and failure data as two separately named files. The

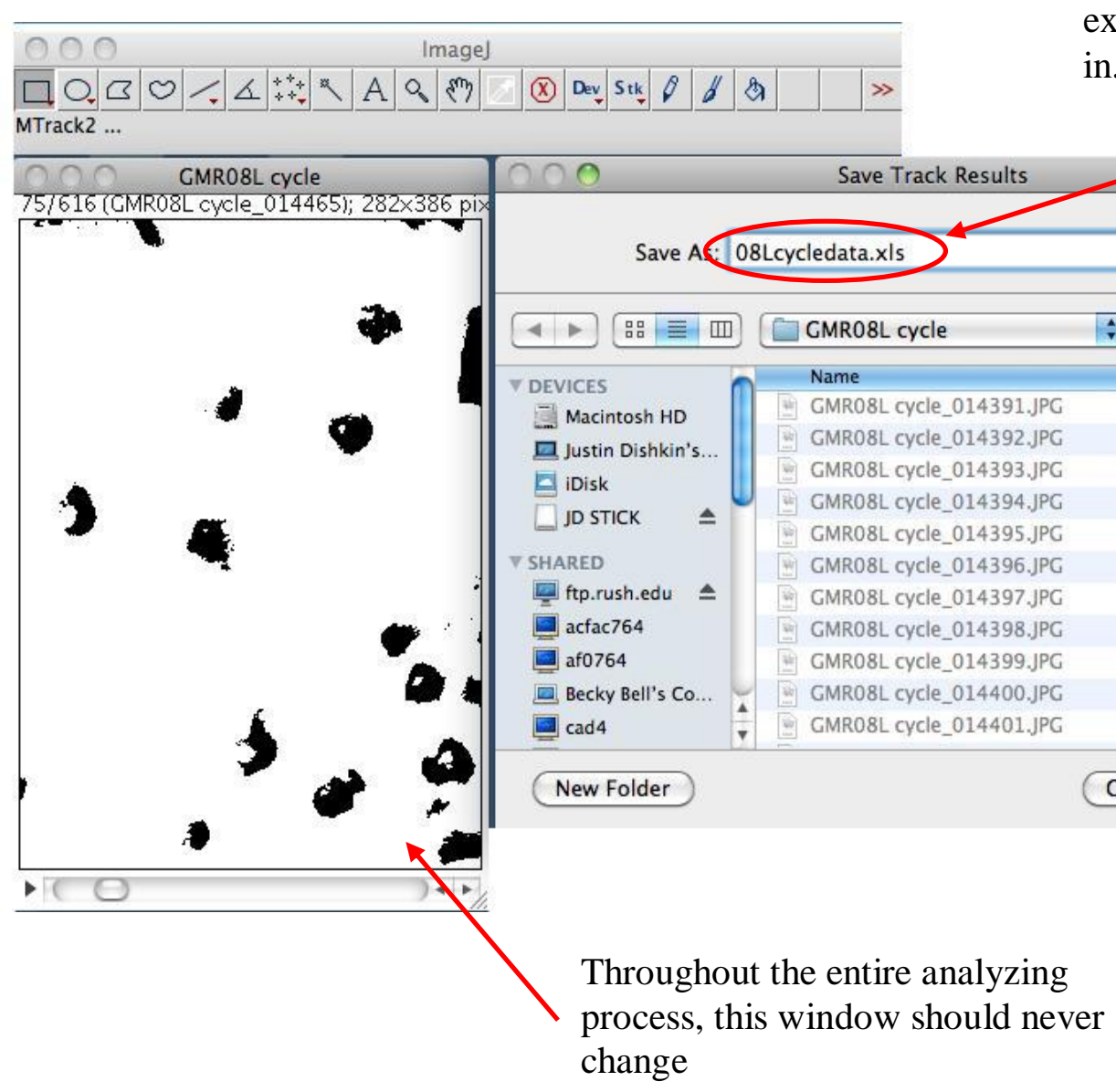
extension needs to be typed in. 


\section{Curve Fitting-MatLab Code for Repair Groups}

This code should be place in a new M-file in Matlab. Save the file with a corresponding name. Then insert the file name in the work space of Matlab along with (Ext,load). This data will come directly from the MTS output.

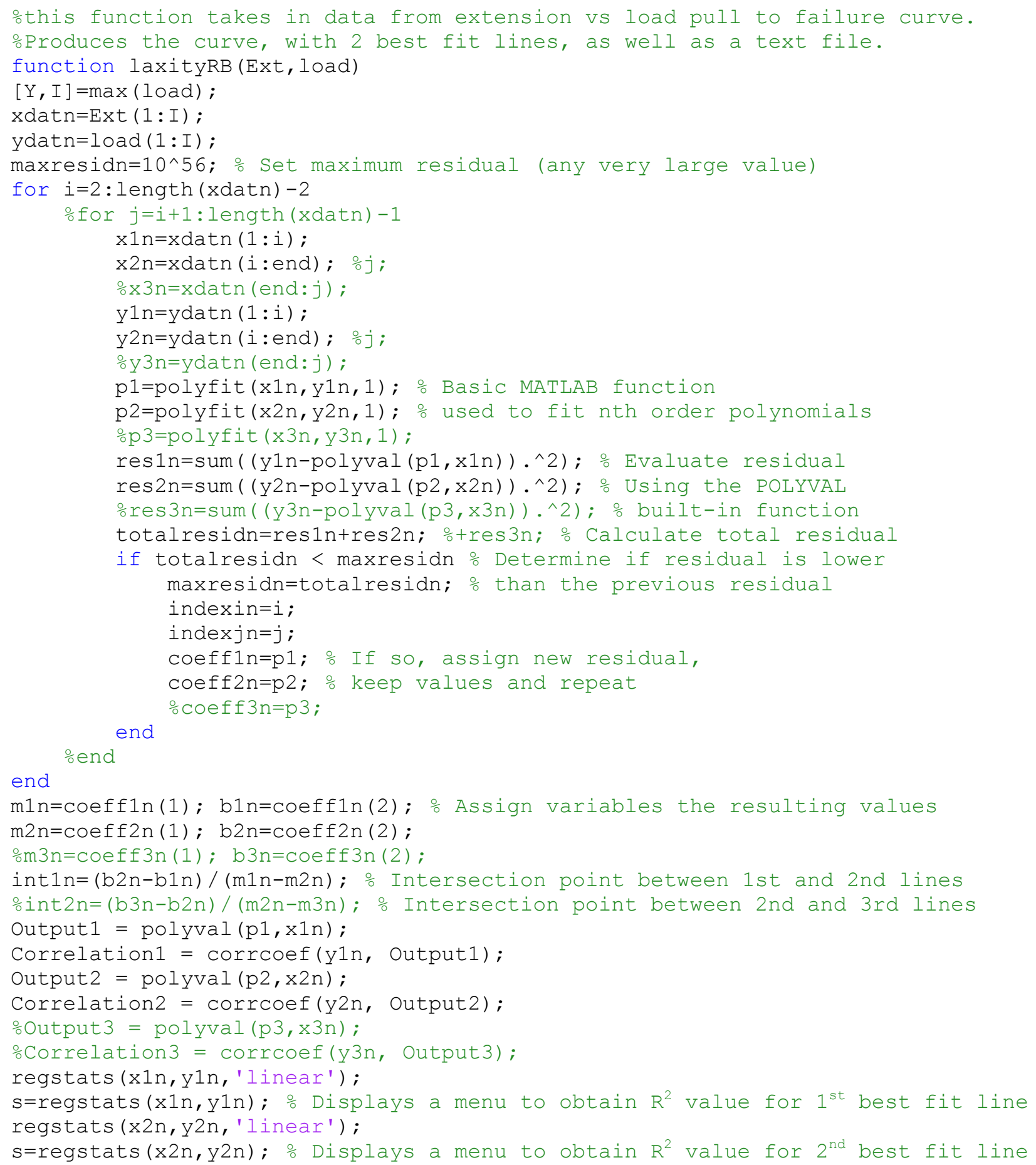




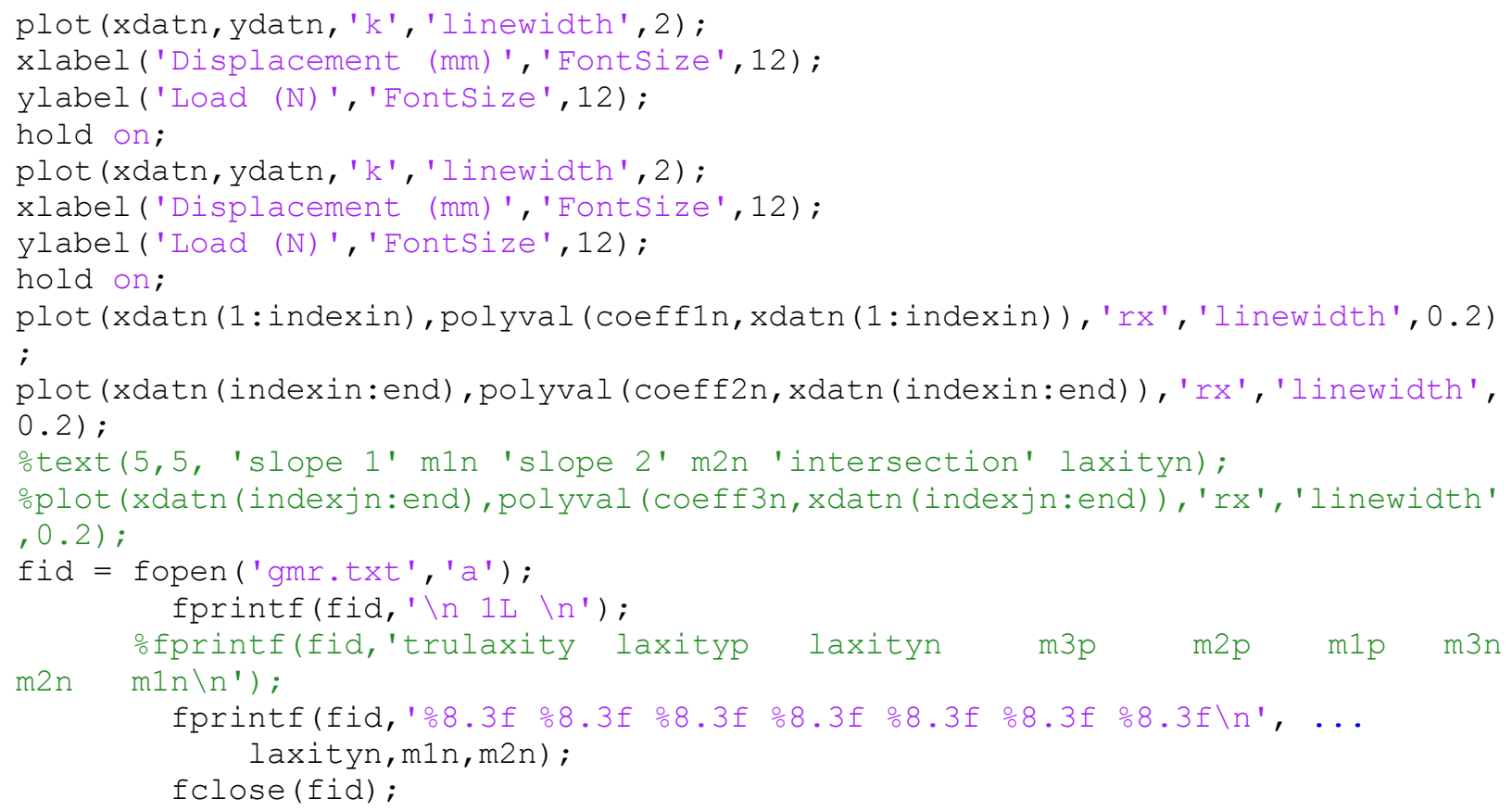

Curve Fitting- MatLab Protocol for Intact Group

1) Select the Load and Displacement measurements from the MTS data in Excel. Convert to Stress and Strain.

2) Paste the data in MatLab with the corresponding labels and use the cftool function.

3) The cftool function will display another screen, where you can select the data to be fitted by pressing the Data button. A new window will appear and after the data is selected press the Create Data Set button and close window. 


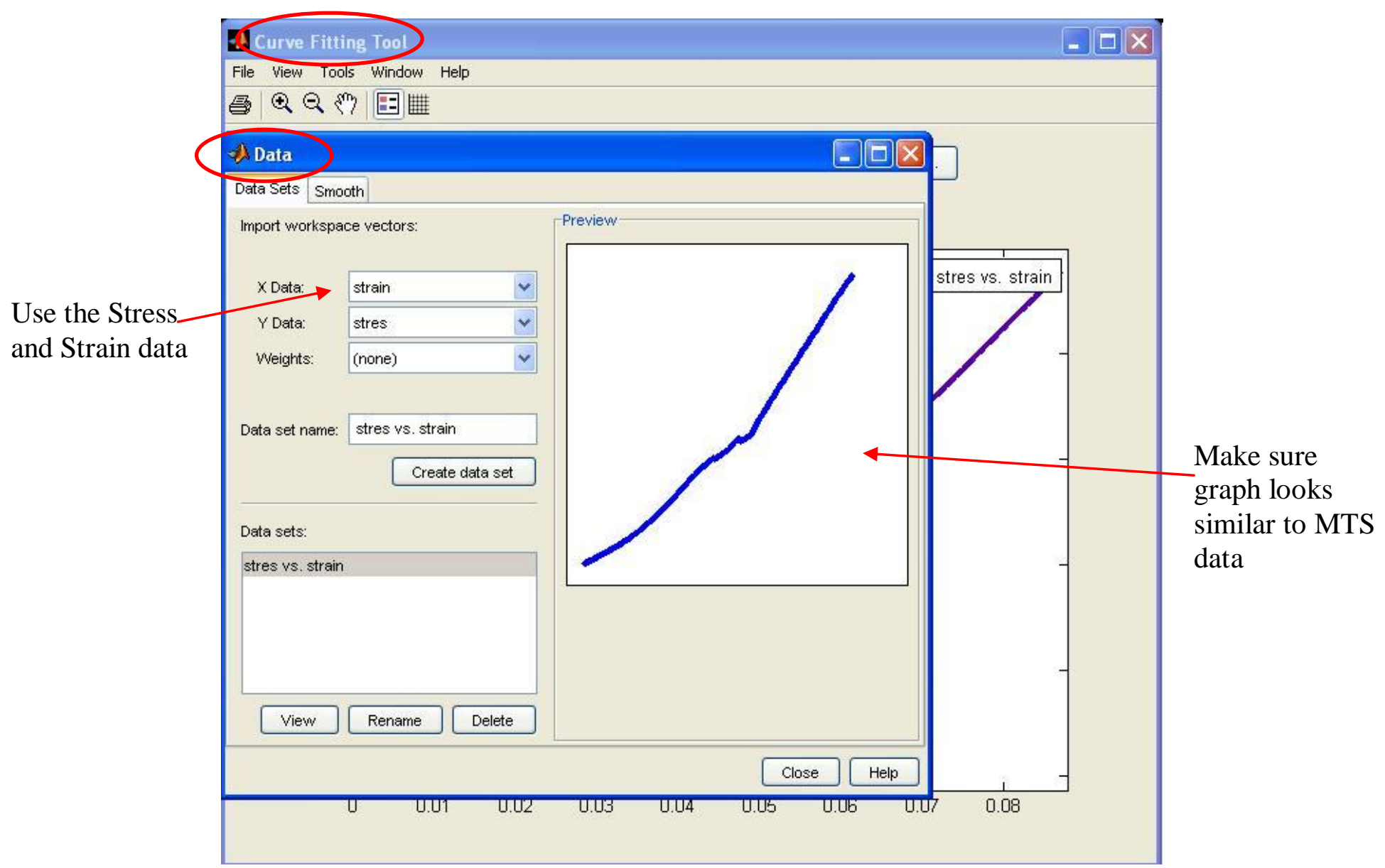

5) Select the Fitting button and a new screen will appear.

6) Data set: Stress vs Strain, Type of Fit: Custom Equations.

7) Select New Equation button and a new screen will appear.

8) In the Create Custom Equation window, select the General Equation tab.

9) Insert the equation, in which the independent variable is Strain and Stress is the y-value. Make sure the equation is correct and parenthesizes are in the correct places.

10) Press OK to close the equation window. Then, press the Apply button to perform fitting. 


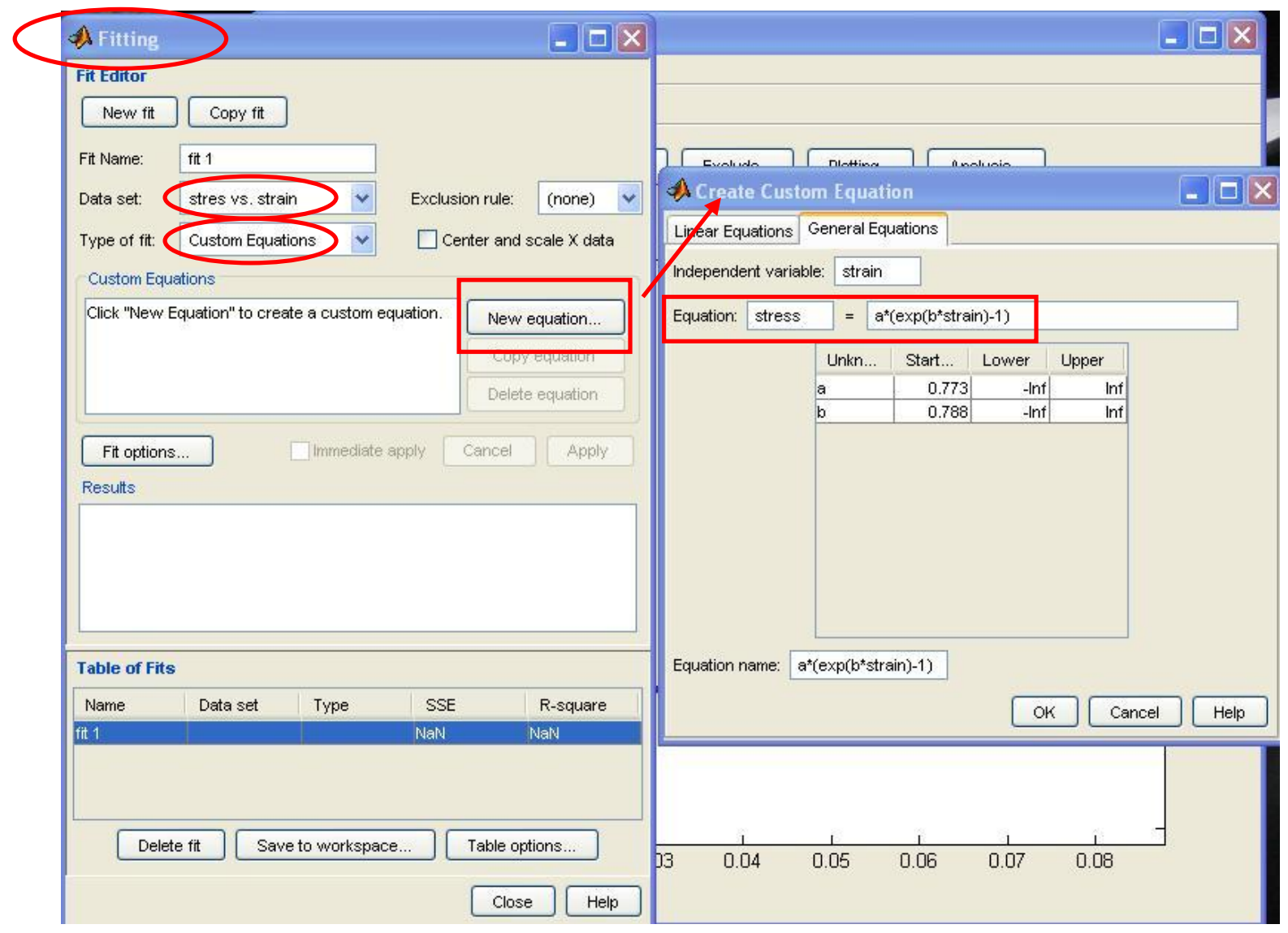

11) The equation will be fitted over the original (blue) data with a red line.

12) In the Results section, the A and $B$ variables will be displayed and a $R^{2}$ value will be calculated to describe the accuracy of the fit.

13) Record the data for each specimen. 


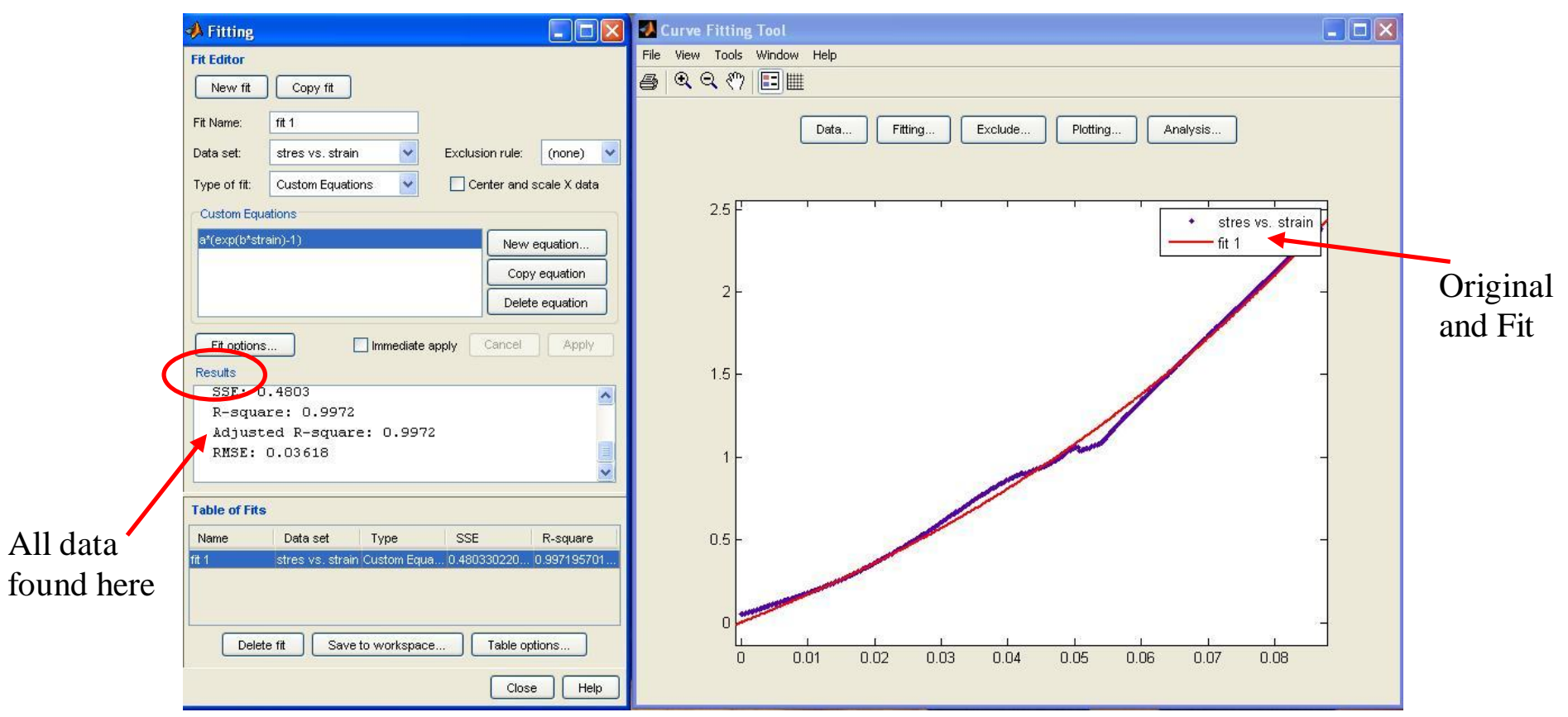




\section{VITA}

NAME: $\quad$ Justin Dishkin-Paset

EDUCATION: $\quad$ B.S.E, Biomedical Engineering, University of Iowa, Iowa City, Iowa, 2009

M.S., Bioengineering, University of Illinois at Chicago, Chicago, Illinois, 2011

PUBLICATIONS: $\quad$ Dishkin-Paset JG, Salata M, Manno, K, Gross C, Shewman EF, Wang VM, Bush-Joseph CA, Nho SJN. "A Biomechanical Comparison of Repair Techniques for Complete Gluteus Medius Tears." manuscript in preparation (American Journal of Sports Medicine).

Dishkin-Paset JG, Ode GE, McArthur SA, Van Thiel GS, Kercher J, Shewman EF, Wang VM, Cole BJ. "Effects of Serial Sectioning and Repair of Radial Tears in the Lateral Meniscus." manuscript in preparation (American Journal of Sports Medicine)

PRESENTATIONS: Rush University Forum for Research and Clinical Investigation. Chicago, IL, USA (April 2010) Ode GE, McArthur S, Van Thiel GS, Kercher J, Dishkin-Paset J, Shewman EF, Wang VM, Cole BJ. "Effects of Serial Sectioning and Repair of Radial Tears in the Lateral Meniscus." Poster

American Orthopedic Society for Sports Medicine. Newport, RI, USA (July 2010). Ode GE, Dishkin-Paset JG, McArthur SA, Van Thiel GS, Kercher J, Shewman EF, Wang VM, Cole BJ. "Effects of Serial Sectioning and Repair of Radial Tears in the Lateral Meniscus." Short Talk

9th World Congress of the International Cartilage Repair Society. Barcelona, Spain (Sept 2010). Ode GE, McArthur S, Van Thiel GS, Kercher J, Dishkin-Paset J, Shewman EF, Wang VM, Cole BJ. Effects of Serial Sectioning and Repair of Radial Tears in the Lateral Meniscus." Poster. 
57th Annual Meeting of the Orthopedic Research Society. Long Beach, California, USA (Jan 2011). Dishkin-Paset JG, Salata M, Manno, K, Gross C, Shewman EF, Wang VM, Bush-Joseph CA, Nho SJ. "A Biomechanical Comparison of Repair Techniques for Complete Gluteus Medius Tears." Poster.

57th Annual Meeting of ORS. Long Beach, California, USA (Jan 2011). Dishkin-Paset JG, Ode GE, McArthur SA, Van Thiel GS, Kercher J, Shewman EF, Wang VM, Cole BJ. "Effects of Serial Sectioning and Repair of Radial Tears in the Lateral Meniscus." Poster and Short Talk.

30th Athroscopy Association of North America Annual Meeting. San Francisco, California, USA (April 2011). Dishkin-Paset JG, Salata M, Manno, K, Gross C, Shewman EF, Wang VM, Bush-Joseph CA, Nho SJ. "A Biomechanical Comparison of Repair Techniques for Complete Gluteus Medius Tears.” Poster

Rush University Forum for Research and Clinical Investigation. Chicago, IL, USA (April 2011). Dishkin-Paset JG, Salata M, Manno, K, Gross C, Shewman EF, Wang VM, Bush-Joseph CA, Nho SJ. "A Biomechanical Comparison of Repair Techniques for Complete Gluteus Medius Tears." Poster 